\title{
MODELING RESULTS FOR A LINEAR SIMULATOR OF A DIVERTOR
}

E.B. Hooper, M.D. Brown, J.A. Byers, T.A. Casper, B.I. Cohen, R.H. Cohen, M.C. Jackson, T.B. Kaiser, A.W. Molvik, W.M. Nevins, D.G. Nilson, L.D. Pearlstein, T.D. Rognlien

June 23,1993

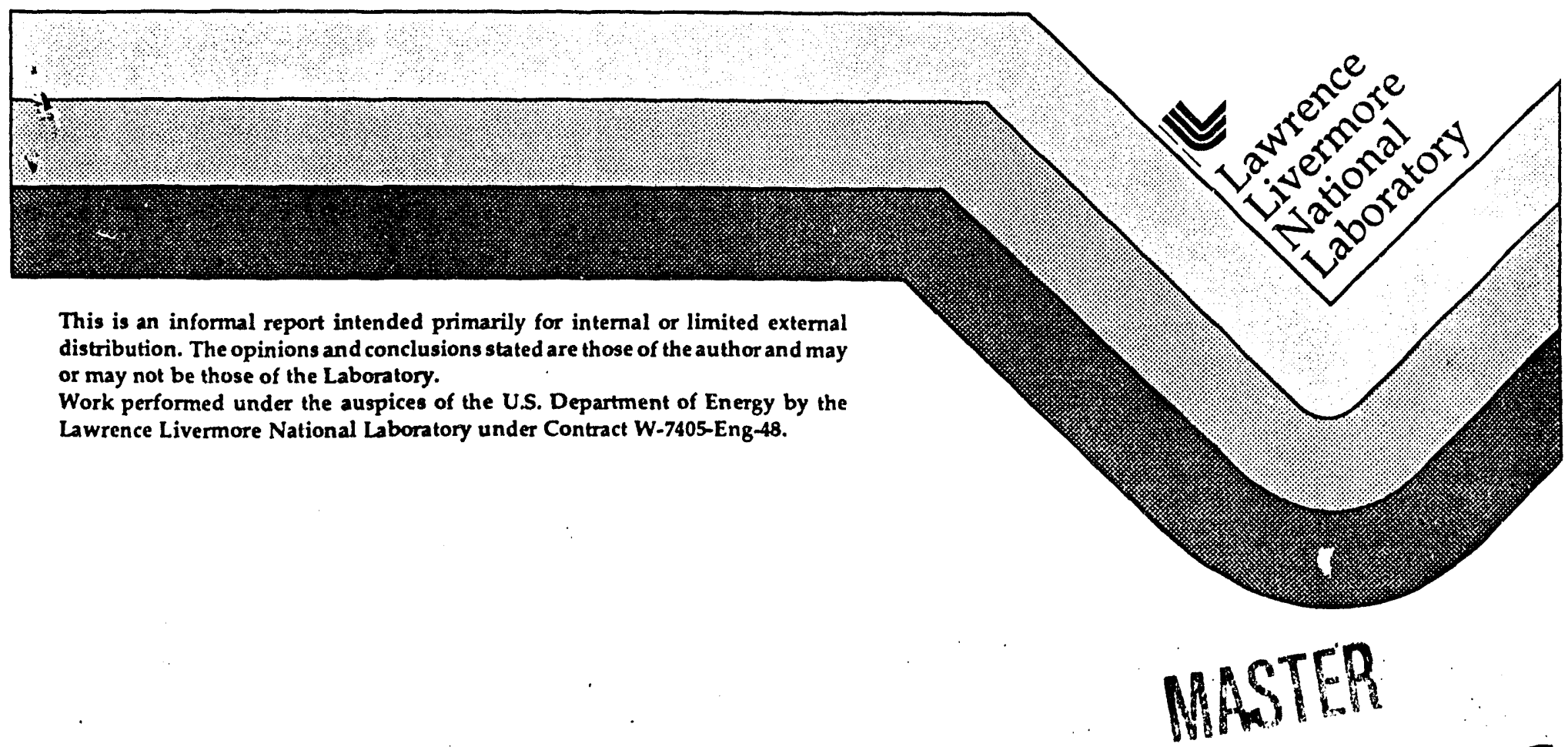




\section{DISCLAIMER}

This document was prepared as an account of work sponsored by an agency of the United States Government. Neither the United States Government nor the University of California nor any of their employees, makes any warranty, express or implied, or assumes any legal liability or responsibility for the accuracy, completeness, or usefulness of any information, apparatus, product, or process disclosed, or represents that its use would not infringe privately owned rights. Reference herein to any specific commercial products, process, or service by trade name, trademark, manufacturer, or otherwise, does not necessarily constitute or imply its endorsentent, recommendation, or favoring by the United States Government or the University of California. The views and opinions of authors expressed herein do not necessarily state or reffect those of the United States Government or the University of California, and shall not be used for advertising or product endorsement purposes.

This report has been reproduced directly from the best arailable copy.

A vailable to DOE and DOE contractors from the Orice of Scientific and Technical Information

P.O. Box 62, Oak Ridge, TN 37831

Prices available from (615) 576-8401, FTS 626-8401

Available to the public from the

National Technical Information Service

US. Department of Commerce 5285 Port Royal Rd,

Springrieled, VA 22161 
TABLE OF CONTENTS

1. Executive Summary

1.1 General Summary

1.2 Specific Conclusions

2. Plasma Heating and Equilibrium

2.1 Introduction

2.2 ICRH: Ion Heating

2.3 Electron Heating by Landau Damping

2.4 Electron Cyclotron Resonance Heating

2.5 Direct Radial Transport due to ICRF

2.6 ICRF Ponderomotive Effects

2.7 Parametric Instability

2.A ICEPIC Simulations of RF Heating and Collisional Coupling

2.B Comparison of Quasilinear Heating Model with Detailed Equation of Motion for Strong RF

3. ICRF Heating of Electrons in IDEAL: Mode Conversion and Landau Damping at the Alfvén Resonance

3.1 Introduction

3.2 $\quad \beta$ Limitation for Effective Interior Electron Landau Heating

3.3 Calculation of Electron Absorption in TMX-U and Phaedrus

3.4 Recent Russian Calculations of Electron Heating for IDEAL

3.5 Modeling Electron Absorption via McVey's ANTENA Code

3.6 Damping from Excitation of Nearly Lossless Modes

3.7 Mode Description

3.8 What Tools do we need for Calculation of Electron Heating?

4. Electron Cyclotron Resonance Heating: Proposed Geometry of Injection

4.1 Summary

4.A Power Deposition by $18 \mathrm{GHz}$ ECH in the TMX-U Central Cell 
5. UEDGE Calculations

5.1 Introduction

5.2 The UEDGE Code

5.3 Geometry, Sources, and Parameters for the Calculations

5.4 Results for Central Fueling

5.5 Results for Edge Fueling with a Radial Limiter

5.6 Summary

6. Experimental Results on Plasma Heating

6.1 Power Accountability in ICRF Heated Mirrors

6.2 Direct Heating of Electrons by ICRF in Mirror Machines

6.3 Bench-marking the ANTENA Code

7. Plasma Startup

8. Limiter Heating

9. Engineering and Cost Estimates

9.1 Engineering Summary

9.2 Benchmark Assumptions

9.3 Benchmark Estimate 


\section{Executive Summary}

A divertor simulator, IDEAL, has been proposed by S. Cohen to study the difficult power-handling requirements of the tokamak program in general and the ITER program in particular. Projections of the power density in the ITER divertor reach $\sim 1 \mathrm{GW} / \mathrm{m}^{2}$ along the magnetic fieldlines and $>10$ $\mathrm{MW} / \mathrm{m}^{2}$ on a surface inclined at a shallow angle to the fieldlines. These power densities are substantially greater than can be handled reliably on the surface, so new techniques are required to reduce the power density to a reasonable level.

Although the divertor physics must be demonstrated in tokamaks, a linear device could contribute to the development because of its flexibility, the easy access to the plasma and to tested components, and long pulse operation (essentially $\mathrm{cw}$ ). However, a decision to build a simulator requires not just the recognition of its programmatic value, but also confidence that it can meet the required parameters at an affordable cost.

Accordingly, as reported here, it was decided to examine the physics of the proposed device, including kinetic effects resulting from the intense heating required to reach the plasma parameters, and to conduct an independent cost estimate. The detailed role of the simulator in a divertor program is not explored in this report.

\subsection{General Summary}

In order to test divertor concepts and hardware, the simulator plasma should carry $\sim 1 \mathrm{GW} / \mathrm{m}^{2}$ of (peak) power in a channel at least $0.01 \mathrm{~m}^{2}$ in area. The plasma density several meters (along fieldlines) from the tested divertor region should be $0.5-5 \times 10^{20} \mathrm{~m}^{-3}$, with ion temperatures of several hundred $\mathrm{eV}$ and electron temperatures $\geq 100 \mathrm{eV}$, sufficient to exceed the radiation barrier for most atomic species. We find that these conditions can be satisfied if both electrons and ions are heated directly at about $50 \mathrm{~kW} / \mathrm{cm}^{2}(\approx 2 \mathrm{MW})$ for each end of the device. At densities below about $1 \times 10^{20} \mathrm{~m}^{-3}$ the electrons and ions are weakly coupled and the power flow in each species is approximately determined by its direct heating. 


\subsection{Specific Conclusions}

1. At densities of interest and power of a few MW, collisional heating of the electrons from ion-cyclotron resonance heated (ICRH) ions will not generate $T_{e}$ above $\sim 50 \mathrm{eV}$. At the low densities, the ions leave the plasma column due to $\mu \nabla B$ forces (in the magnetic beach) before they transfer significant energy to the electrons. At higher densities, there are too many electrons to achieve high $T_{e}$ in the presence of Spitzer thermal conductivity to the ("divertor") end plate.

2. Direct electron heating can be achieved by Landau damping of the low frequency (ICRH) wave or by electron cyclotron resonance heating (ECRH). Calculations show that either mechanism will generate $T_{e}=100 \mathrm{eV}$ despite the lack of magnetic confinement.

3. At the lower densities of interest, the power flow in electrons and ions can be independently varied.

4. Additional electron heating may occur if the kinetic Alfvén cyclotron wave is excited at the Alfvén resonance and subsequently Landau damps in the plasma core. We have identified the calculations required to evaluate this possibility but have not carried them out.

5. ECRH can be applied by injection of the $X$-mode at frequencies below the cyclotron resonance. The wave is predicted to be trapped by radial density gradients and guided to the resonance where it is absorbed.

6. Parametric instabilities are not expected to cause serious limitations despite the intense $\mathrm{rf}$ waves at the required power densities.

7. 2-D fluid calculations demonstrate a high sensitivity to the recycling (fueling) of the plasma at the "divertor" plate. In some cases the plasma is found to have a high-temperature, low-density ("low recycling") core surrounded by a low-temperature, high-density ("high recycling")region. 'This effect arises in part due to the effective radial transport resulting from recycled neutrals leaving the end plate at an angle to the magnetic field. The effect may be enhanced by poor penetration of neutrals in the gas box, assumed located at the center plane of the device.

8. Limiter heating is potentially very high and needs considerable attention. Both shaping, with the fieldlines nearly tangential to materials surfaces, and gas pumping to minimize plasma outside the primary flux tube may be required to prevent limiter damage. 
9. A review of ICRH experiments in mirror machines finds power accountabilities ranging from $65 \%$ to $\geq 73 \%$. In some cases, direct elect:on heating was demonstrated to dominate drag heating by a factor of 4 . Losses due to charge exchange should be less in IDEAL as the gas box, located at the midplane, is isolated from the hot ions by the $\mu \nabla B$ force associated with the magnetic beach. In addition, radial transport effect should be reduced as the ions in the unconfined (flowing) plasma are lost before moving radially an appreciable distance. However, heating of the limiter must be of concern, as noted above.

10. Plasma startup could be achieved by several methods. Easiest appears to use a plasma gun which can be extracted from the column in $<1$ second.

11. The cost of IDEAL as designed is estimated to be roughly $\$ 45 \mathrm{M}$, assuming no site credits except a building, manpower costed using PACE rules, and site "taxes" only of $9.7 \%$ on purchased items. Accordingly, $\$ 10 \mathrm{M}-\$ 15 \mathrm{M}$ of site credits will be required to meet a $\$ 30 \mathrm{M}-\$ 35 \mathrm{M}$ budget unless the cost can be reduced, e.g. through design modifications. Design and construction in 30 months appears feasible if funding is available. 


\section{PLASMA HEATING AND EQUILIBIUM}

E. B. Hooper, B. I. Cohen, M. D. Brown, and T. D. Rognlien

\subsection{Introduction}

Ion cyclotron resonance heating (ICRH) by slow waves changes the perpendicular energy at the Doppler-shifted resonance thus generating a tail along corresponding characteristics in velocity space. This tail may, in general, extend to high energy. Furthermore, in a magnetic configuration consisting only of a magnetic hill with no mirror section for confinement, the perpendicular motion is converted into motion along the magnetic field. The energetic particles may, therefore, be lost from the system before being thermalized or losing appreciable energy by drag on the electrons. Eviluation of the effects of the heating therefore must include kinetic effects. We have made such an evaluation using a particle-in-cell computer code, ICEPIC. The code is briefly described in Appendix 2.A to this chapter.

We will see that it is difficult in this magnetic configuration to heat the electrons to energies above $40-50 \mathrm{eV}$ at densities of $0.5-2 \times 10^{20} \mathrm{~m}^{-3}$ by collisions with energetic ions generated by a few MW of ICRH in hydrogen plasmas $\left(Z_{\text {eff }}=1\right)$. Generally, at the lower densities, the energetic ions are lost before transfering their energy to the electrons; at the higher densities where the slowing down time of the ions on the electrons is shorter than the loss time, the energy available is too low to heat the electrons to a higher temperature.

If electron temperatures approaching $100 \mathrm{eV}$ are desired in a linear divertor simulator such as IDEAL, it is necessary to have direct electron heating or to apply the ion heating in a mirror cell. Direct electron heating can be obtained from Landau damping due to the (small) longitudinal electric field component of the slow wave, from damping of kinetic waves excited by the slow wave, or direct electron cyclotron resonance heating. Landau damping and ECRH are modeled in the ICEPIC code, and results are presented here. The effects of the kinetic Alfvén cyclotron wave are considered separately, and the critical issues determined. Quantitative evaluation of the resultant wave generation and heating will require detailed analysis.

Ion heating at multiple frequencies is demonstrated. The diffusive nature of the heating process permits some leakage of ions up the magnetic hill from each resonance, so that each heating zone is fueled. 
We also examine parametric instabilities and radial transport during heating; we predict that neither will be of major importance. Ponderomotive effects may play an important role in the plasma equilibrium.

Heating in a mirror cell offers an interesting option. In particular, the long residence time of the energetic ions permits significant energy transfer to the electrons. It might be possible to control the fraction of energy converted to electron heating by tuning the strength of the magnetic mirror. The equilibria are quite different from those analyzed here, and new physics issues such as microstability become important. Accordingly, this option is not discussed in this report.

\subsection{ICRH: Ion Heating}

2.2.1 Introduction. The magnetic field strength is an approximation to the nominal field design for IDEAL, varying from $3.5 \mathrm{~T}$ upstream of the magnetic beach to $2.5 \mathrm{~T}$ downstream; see Fig. 2.1 for the "base-case" field used in the modeling. As the calculation is one-dimensional in space, quadrupole effects are not included.

Figure 2.1 also shows the fueling locations used in the calculation. In actual code running, the fueling rate is specified (nominally with $R=0.9$ ), and the resultant equilibrium determined.

The left-hand boundary $(z=0)$ is a symmetry point; the right-hand boundary is an absorbing wall. In the latter case, charges lost to the wall are accumulated to ensure time-averaged quasineutrality throughout the plasma.

To calculate absorption, the wave is considered to propagate in the high-field region with a typical parallel wave vector magnitude $0.43 \mathrm{~cm}^{-1}$. Slow wave heating in the ICEPIC code is modeled by quasilinear diffusion in perpendicular energy at the ion Doppler-shifted cyclotron resonance. As the heating occurs very close to the cold cyclotron resonance for all heated ions, the variation in the magnitude of the wave vector is neglected for ICRH. In addition, as the resonance is approached, the rf electric field in the actual plasma increases as the group velocity approaches zero but decreases as the power is absorbed. This latter calculation would require a lengthy selfconsistent calculation which was not attempted here. We therefore neglect these variations and adjust the rf electric field to obtain the desired absorbed power.

Both ions and electrons are followed in the ICEPIC code. In order to obtain results in a reasonable computational time, the density is decreased by a factor of $10^{7}$ for the present calculations. (In the code proper, only about $10^{4}$ 


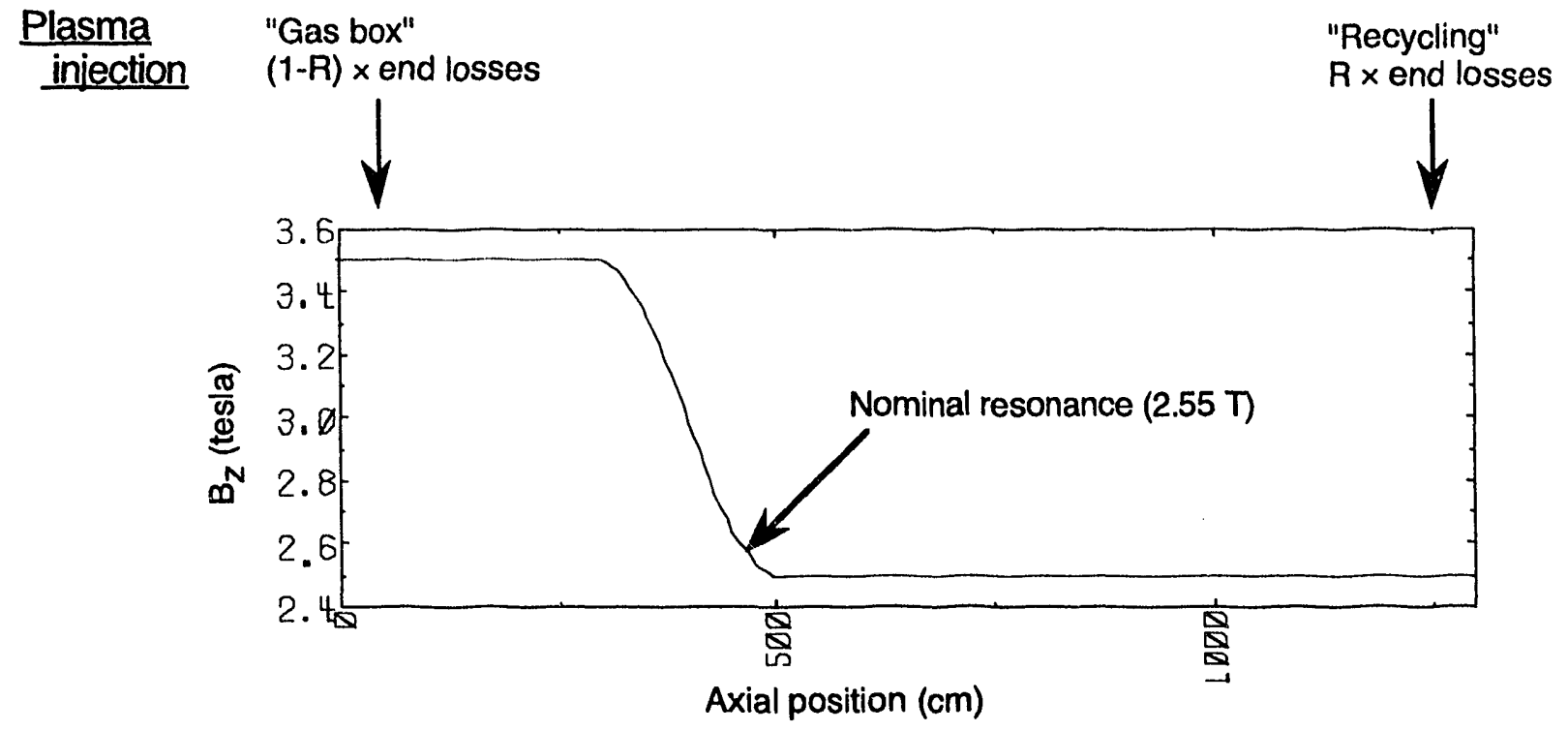

Figure 2.1. Magnetic field and fueling locations. The field is fit by a cubic spline between 3.5 and $2.5 \mathrm{~T}$.

"superparticles" are followed.) Collisions are increased by the same factor to yield the proper collision frequency. A sheath develops to maintain quasineutrality in the plasma, although its structure is not accurately determined as Debye length effects are poorly resolved.

Calculations with the correct electron-ion mass ratio are still time consuming. Accordingly, many of the results are obtained using "heavy electrons," with a mass 18.36 times the true electron mass. The slowing down rate for electrons on ions is proportional to $\left(m_{e} / m_{i}\right) / \tau_{e} \sim m_{e}{ }^{1 / 2}$, with $\tau_{e}$ the electron collision time. The dominant electron cooling is due to Spitzer thermal conductivity along the magnetic field, with a cooling rate also proportional to $m_{e}{ }^{1 / 2}$. To keep the power balance approximately correct, the electron-electron and electron-ion collision frequencies are reduced by $(18.36)^{-1 / 2}$. Electron velocities are slow compared to the actual case, and so the corrections only approximate the actual mass case. Calculations using the actual electron-ion mass ratio yield electron temperatures about $20-25 \%$ less than those obtained in the equivalent heavy-electron cases.

2.2.2 Ion Heating results. As the ions are heated on a magnetic hill, the conversion of perpendicular to parallel energy through the $\mu \partial B / \partial s$ force will cause a flow loss to the end wall. This loss limits the residence time of the ions in the plasma column and thus the transfer of energy to electrons and 
cold ions. To minimize the effect, we heat near the bottom of the beach, at $B=2.55 \mathrm{~T}$, corresponding to heating at $37.7 \mathrm{MHz}$; the necessary frequency scales with $B$. Figure 2.2 shows the equilibrium resulting from a heating power of $5.6 \times 10^{4}$ watts $/ \mathrm{cm}^{2}$ per end (referenced to $3.5 \mathrm{~T}$ where the antennas are located). The equivalent whole column power is approximately $2 \times 2.2 \mathrm{MW}$. The "temperatures" are actually moments of the distributions; for ions, especially, the distribution is highly non-Maxwellian.

The plasma shown has a recycling coefficient of 0.9 in the form of a cold plasma $\left(T_{e}=T_{i}=5 \mathrm{eV}\right)$ injected near the divertor plate end of the column. The remainder of the fueling is cold plasma injected near the center of the column $(z=0)$. This plasma injection is at a rate $S=6.64 \times 10^{19}$ ion-electron pairs $/ \mathrm{cm}^{2}$-s. (If injected in a gas box, this corresponds to $0.9 \mathrm{~T} \ell / \mathrm{s} \mathrm{of} \mathrm{H}_{2}$ for each $\mathrm{cm}^{2}$ of plasma.) This injection rate would be reduced considerably if larger recycling coefficients occur in the actual device.

The ion heating is almost entirely single pass absorption. The antennas are assumed to be in the high field region at $z<z$ (resonance), and the waves propagate to the resonant zone; for the half-space shown, this corresponds to propagation in the $+z$ direction. Ions are heated at their Doppler-shifted resonance, so those traveling in the $+z$ direction are heated at fields below the resonance and those traveling in the $-z$ direction at fields above the resonance. However, the slow wave does not propagate at fields below the resonance, at least in the cold-plasma approximation. Consequently, only ions traveling in the $-z$ direction are heated; these arise primarily from recycling at the divertor plate.

The heated ions will generally receive sufficient perpendicular energy that they will turn on the magnetic hill and be "pushed" towards the divertor plate. This effect results in a density drop at the heating location, as observed in Fig. 2.2. The effect is balanced somewhat by the electric potential which develops to maintain charge neutrality and reflects cold ions from the gas box back towards the origin.

The ion distribution is highly anisotropic, as shown in Figs. 2.3 and 2.4. The ion distribution function is shown in three regions corresponding to the high field side of the configuration, just downstream from the resonance, and near the divertor plate. The distribution has relaxed somewhat, primarily by drag on the electrons, as seen in the contour plots and the distributions. The extent of the relaxation can be quantified from the energy fluxes, $Q$, shown in 

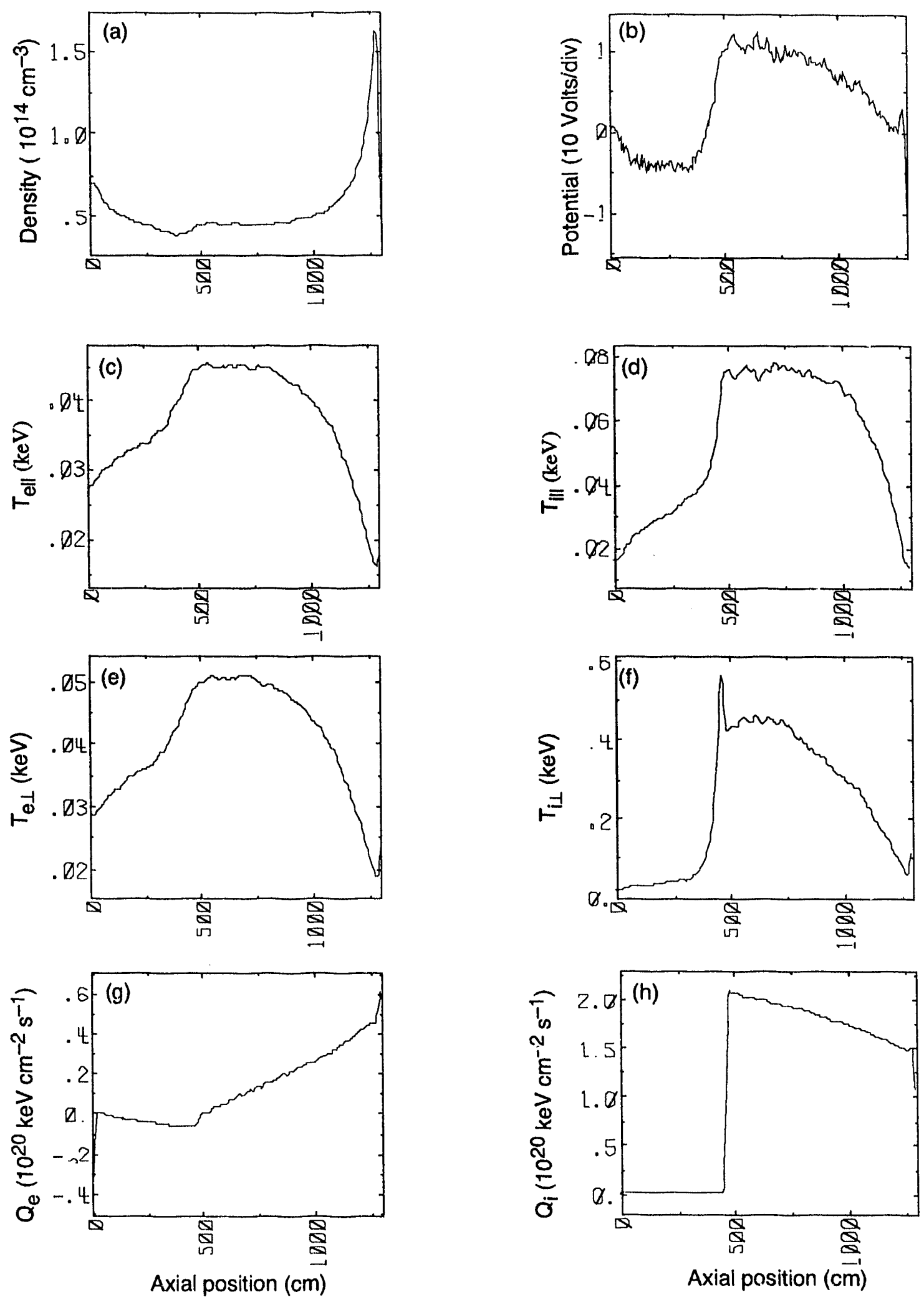

Figure 2.2. ICRH for $P_{I C R H}=56 \mathrm{~kW} / \mathrm{cm}^{2}, S=6.64 \times 10^{19}$ ion-electron pairs $/ \mathrm{cm}^{2}$-s (both per end); case dv20. No direct electron heating. Electron mass $=18.36$ times true mass. (a) Density, (b) electric potential, (c) $T_{\text {ell, }}$ (d) $T_{i \|}$ (e) $T_{e \perp}$, (f) $T_{i \perp}$, (g) Electron heat flux, (h) Ion heat flux. 
Fig. 2.2. Note that the ion energy flux drops only by about $20 \%$ from the heating near $z=500 \mathrm{~cm}$ to the divertor end. Indeed, the ion heat flux at a position just before the sheath is $1.8 \times 10^{20} \mathrm{keV} /\left(\mathrm{cm}^{2}-\mathrm{sec}\right)=2.9 \times 10^{4} \mathrm{~W} / \mathrm{cm}^{2}$, and the electron flux is $0.5 \times 10^{20} \mathrm{keV} /\left(\mathrm{cm}^{2}-\mathrm{s}\right)=0.8 \times 10^{4} \mathrm{~W} / \mathrm{cm}^{2}$. (Multiply these by the flux ratio, 3.5/2.5, to compare with the applied heating.) The ions thus carry most of the heat to the end of the device for these plasma conditions.

Heating further up the magnetic hill decreases the coupling further consistant with the larger change in parallel energy as the ions are "pushed" down the field gradient. The parallel velocity of an ion is increased by $\mu \Delta B$; thus ions neated at $B=3 \mathrm{~T}$ have a resonance time in the column about half those heated at $B=2.55 \mathrm{~T}$. Code runs show that heating at $B=3.0 \mathrm{~T}$ decreases the coupling to about $10 \%$, in agreement with the above estimate.

The coupling can be increased by heating at higher plasma densities resulking from larger plasma sources. Figs. 2.5-2.7 show results of heating at a fueling rate of $1.33 \times 10^{20} \mathrm{~cm}^{-2} \mathrm{sec}^{-1}$, twice that of the case discussed above. The energy transfer to the electrons has increased to about $55 \%$, but the electron temperature has changed only slightly because the energy is shared ar.ong the larger number of electrons. Figure 2.8 demonstrates the scaling of several parameters with the fueling rate. The electron temperature was insensitive to the fueling rate for the given ICRH power. The mass correction for $T_{e}$ indicated in the figure caption was determined from "spot check" runs. 

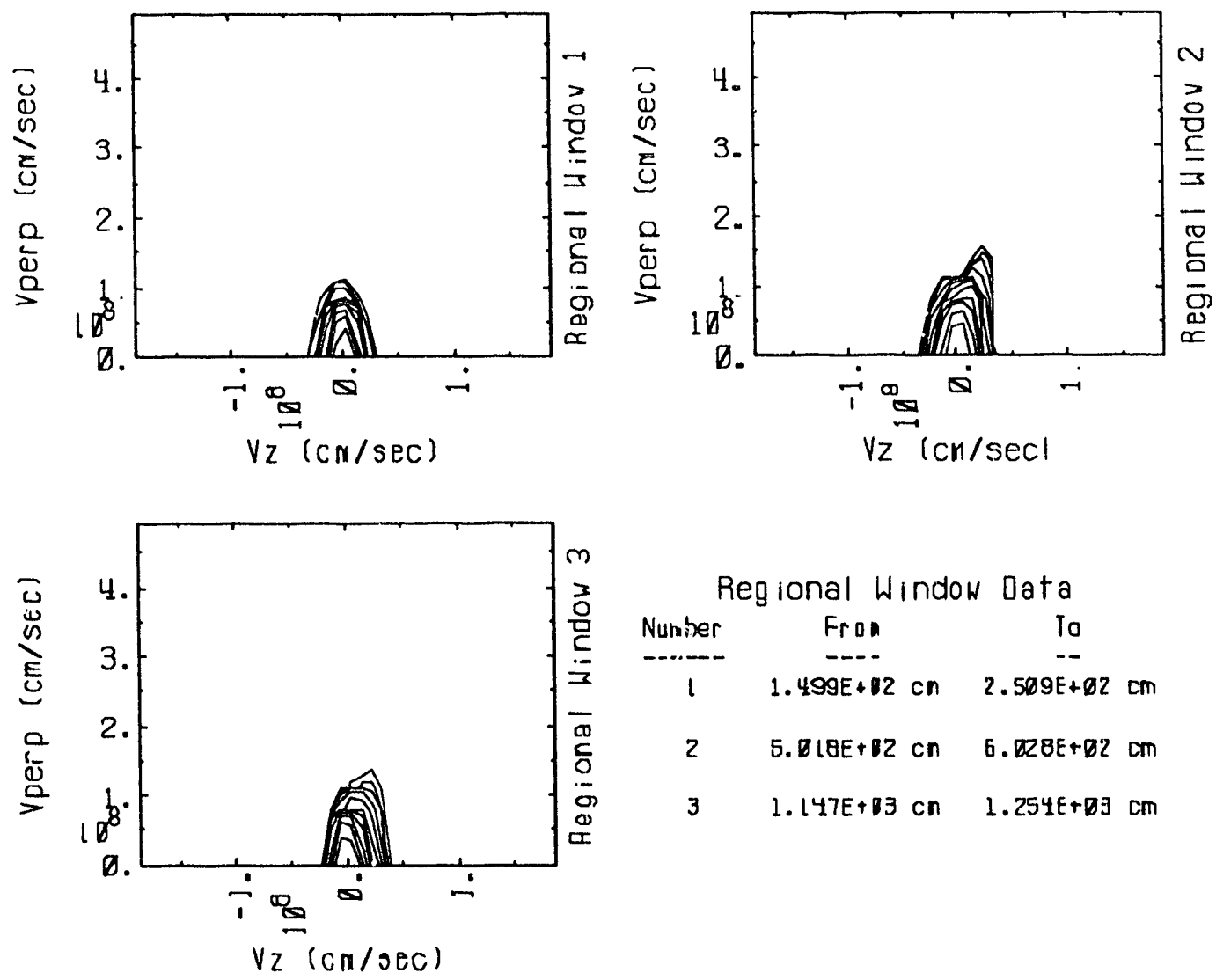

Figure 2.3. Ion velocity contours for the same case (dv20) as Fig. 2.2. The ICRH is applied at $477 \mathrm{~cm}$. 

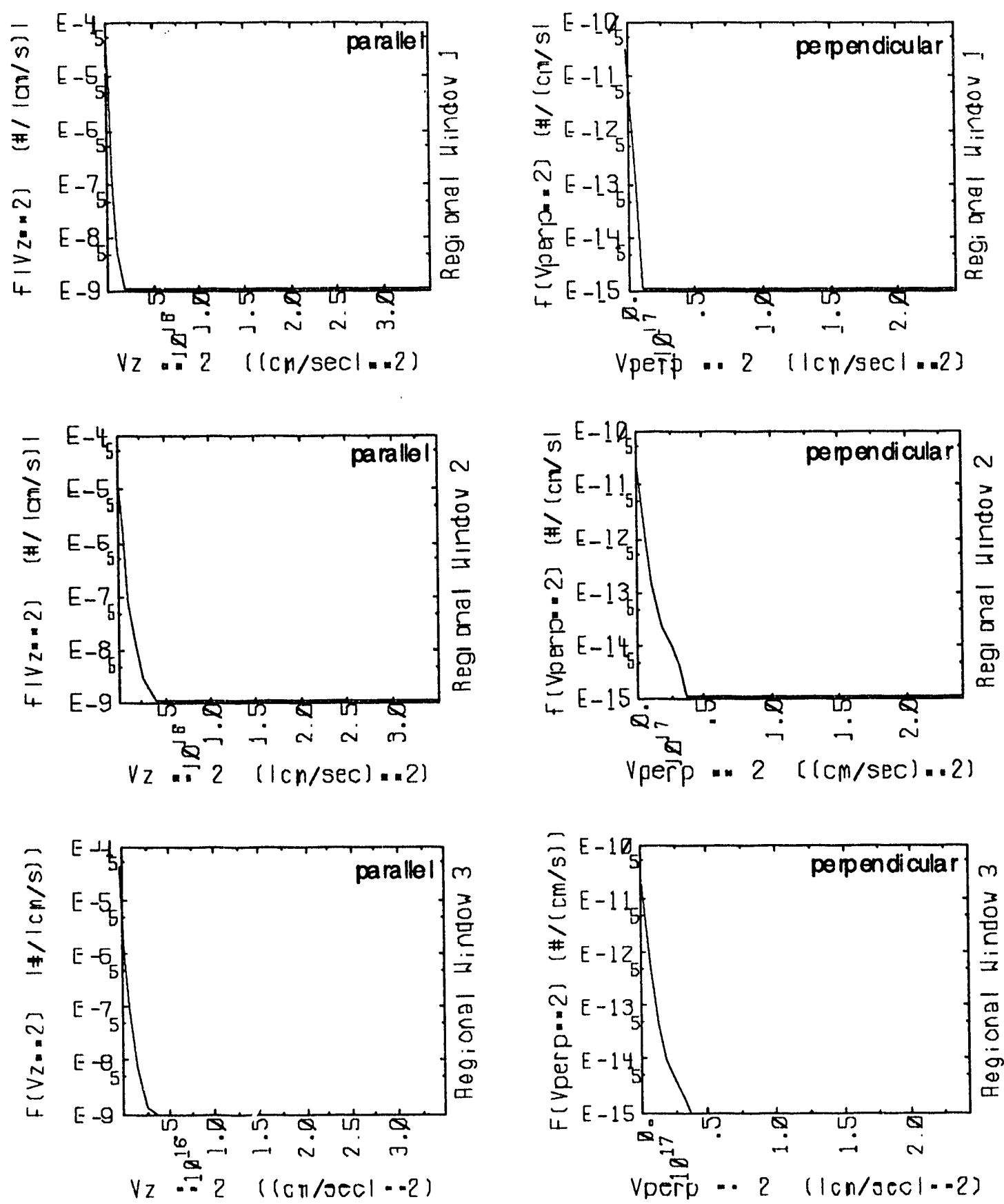

Figure 2.4. Ion distribution functions. Thethree windows correspond to $z=$ $150-250 \mathrm{~cm}, 500-600 \mathrm{~cm}$, and $1150-1250 \mathrm{~cm}$. The heating zone is at $z=477 \mathrm{~cm}$. (Case d v20) 

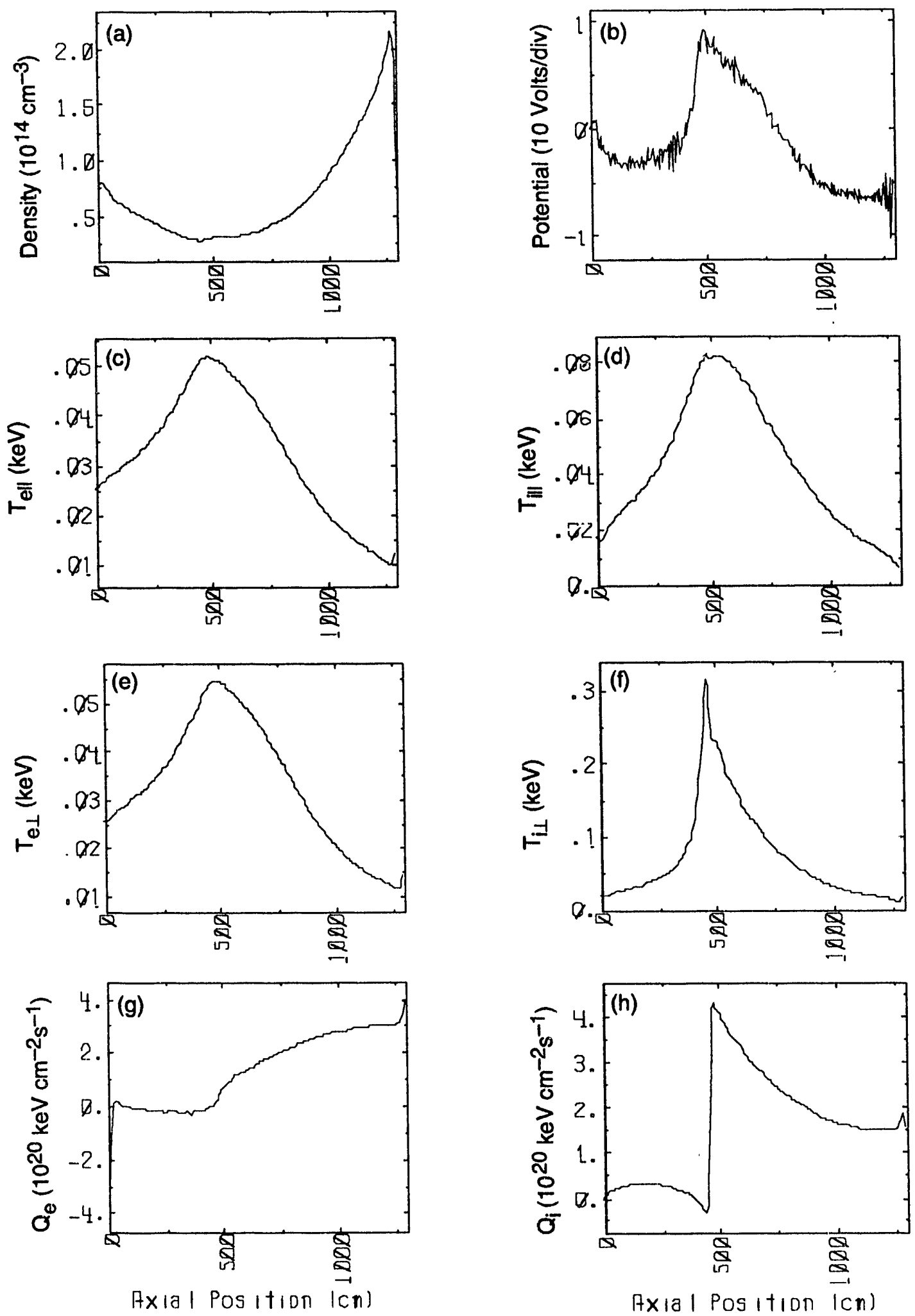

Figure 2.5. $\mathrm{P}_{\mathrm{ICRH}}=110 \mathrm{~kW}, \mathrm{~S}=1.66 \times 10^{20}$ ion-electron pairs $/ \mathrm{cm}^{2}$-s; case dv31. Electron mass $=18.36$ times true mass. Plots as in Fig. 2.2. 

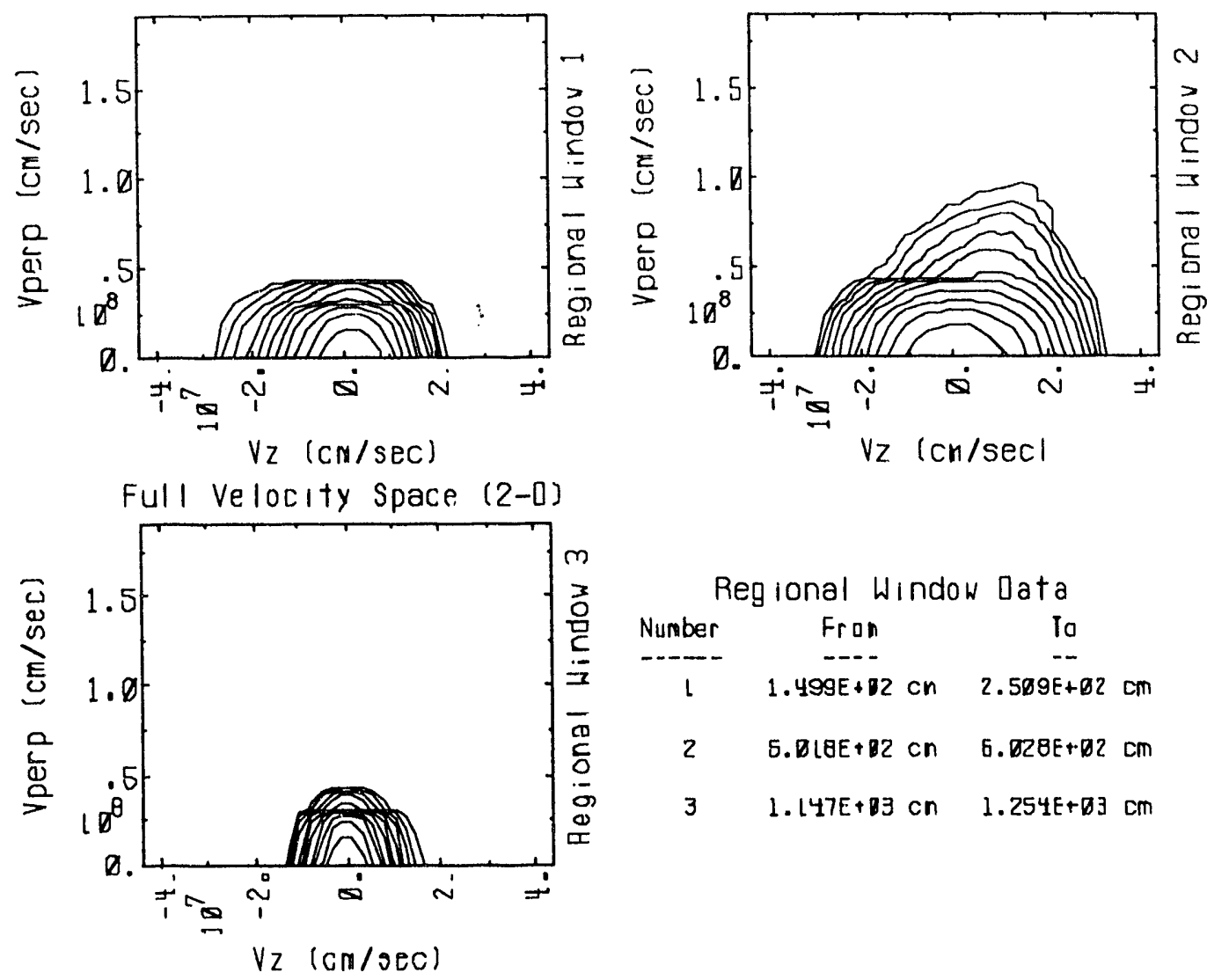

Figure 2.6. Ion velocity contours for the same case as Fig. 2.5. 

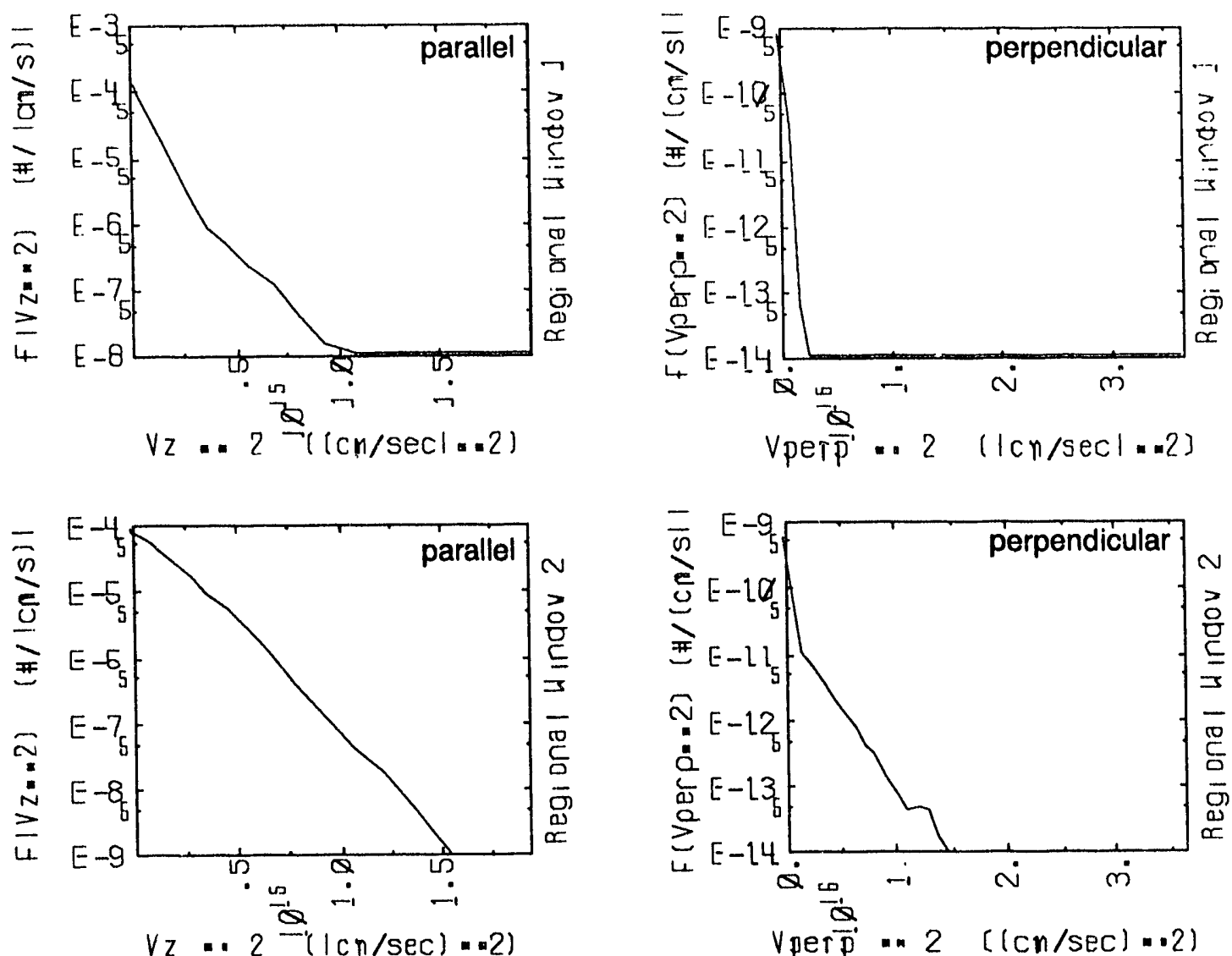

Vper $\vec{p}$. 2 ( (cn/sec) - .2)
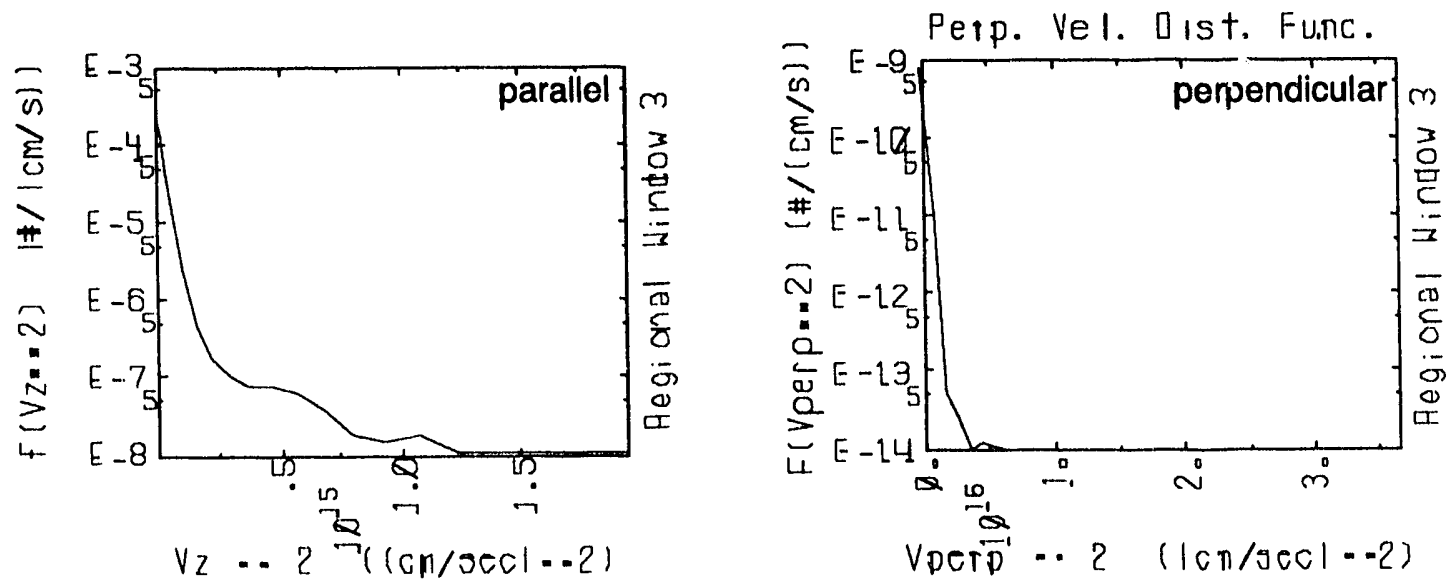

Figure 2.7. Ion distribution functions for case dv31. Regions as in Fig. 2.6 

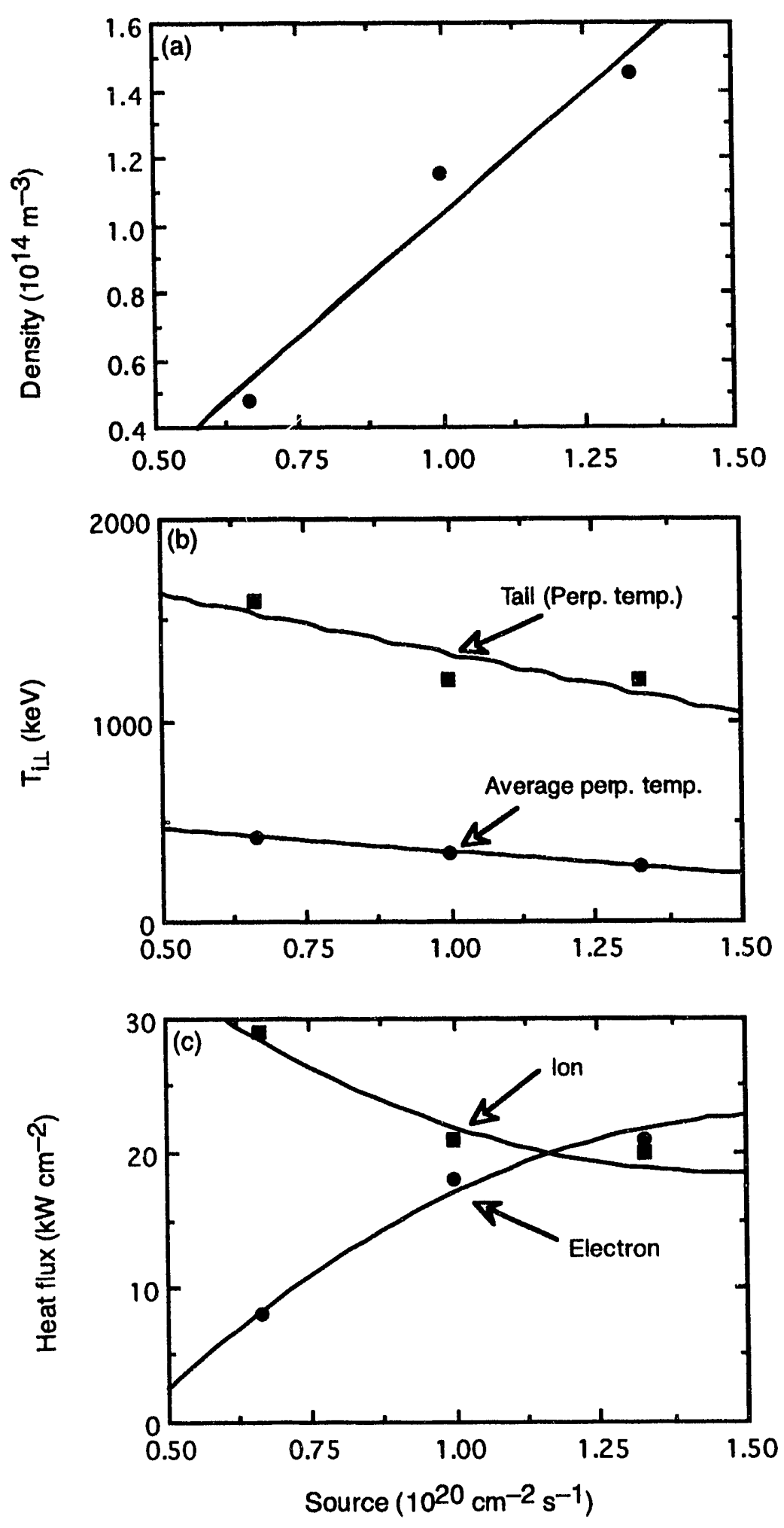

Figure 2.8. Scaling of plasma parameters with source strength: (a) Density, (b) Ion temperature, (c) Heat flux. Runs with heavy electrons ( $\mathrm{m}_{\mathrm{e}}$ increased by 18.36), $T_{e}=50 \mathrm{eV}$; corrected for mass $T_{e} \approx 37 \mathrm{eV} ; P_{I C R H}=60 \mathrm{~kW} / \mathrm{cm}^{2}$. 
The electron temperature is a weak function of the ICRH power. Figure 2.9 shows an increase in $T_{e}$ of a factor of 1.4 for an ICRH power increase of 4.1. Note that the electron temperatures in the figure need to be multiplied by aproximately 0.75 to correct for the electron mass.

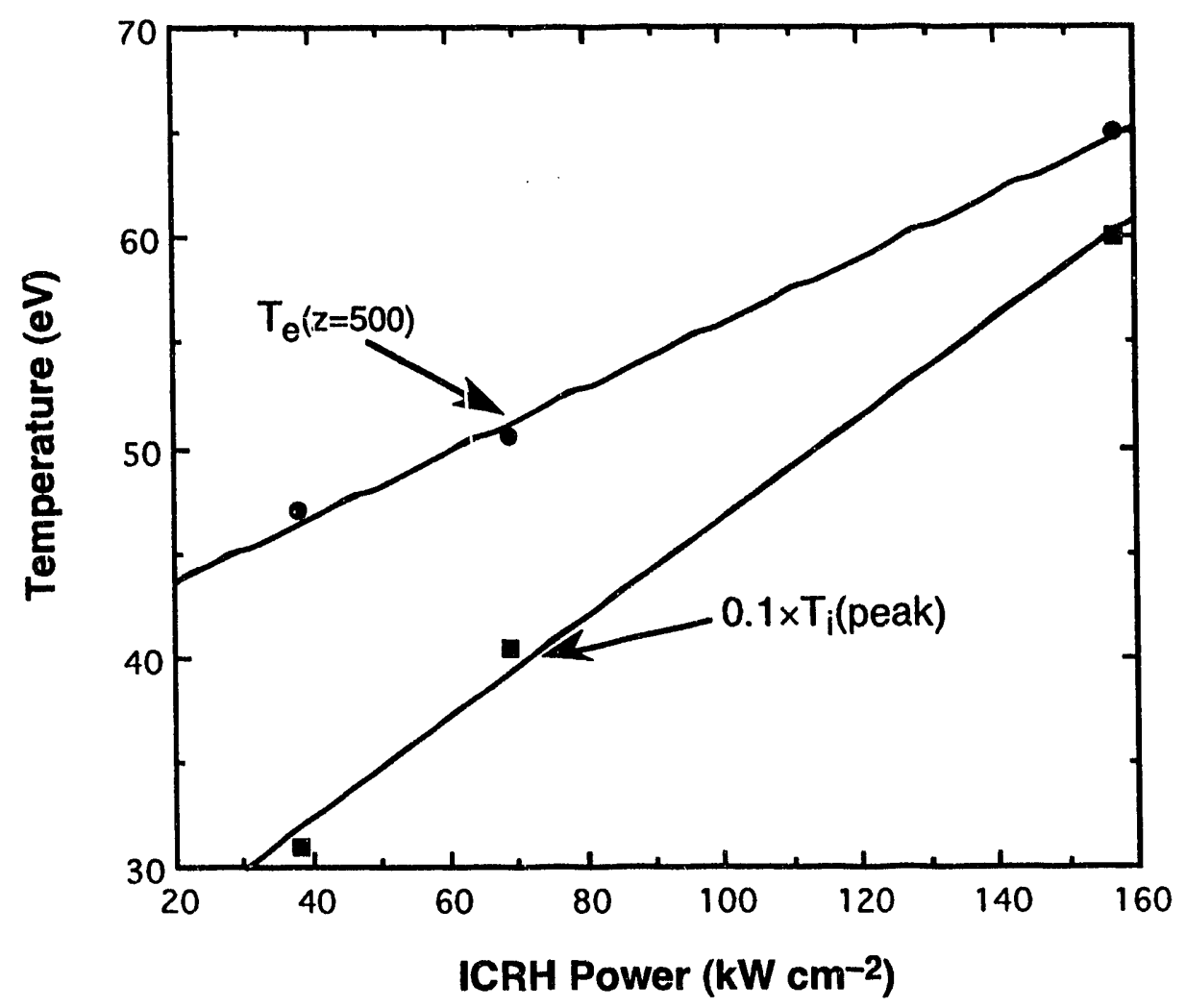

Figure 2.9. Temperatures vs ICRH Power. The source is constant: $S(0)=$ $1.0 \times 10^{20} \mathrm{~cm}^{-2} \mathrm{~s}^{-1}$. The scaling is approximately $T_{e}=P \alpha$, with $\alpha=0.20-0.25$. Temperatures are not corrected for the heavy electron mass $(\times 18.36)$.

2.2.3 Ion heating at multiple frequencies. As there are more than one ICRH antenna. on the proposed device, more than one frequency can be applied, resulting in heating at multiple locations on the magnetic beach. The ability to heat ions is limited, however, as most of the plasma fueling arises from recycling at the divertor plate. As a result, ions which are heated at the resonance at the lowest magnetic field will receive an increase in perpendicular energy which (generally) will cause them to turn on the magnetic gradient. However, as the heating process is diffusive in nature, there is considerable "leakage" through each resonance. The effect is 
demonstrated in Figs. 2.10-2.11. Heating was done at 1, 2, and 3 frequencies, with essentially the same total power. The rf electric fields for the 2 and 3 frequency case were obtained from the single frequence case by scaling by $(2)^{1 / 2}$ and (3) ${ }^{1 / 2}$, corrected by the magnetic field strength at each resonance. Note from the ion heat flux, Fig. 2.10, that the energy absorbed at each resonance is approximately independent of the resonance location; this was confirmed by other code diagnostics.

The highest frequency, $\omega=3.31 \times 10^{8} \mathrm{~s}^{-1}$, was only slightly below the resonance at the central magnetic field, $\omega=3.35 \times 10^{8} \mathrm{~s}^{-1}$. The difference, $4.3 \times 10^{6} \mathrm{~s}^{-1}$ corresponds to a Doppler shifted resonance for ions of velocity $-1 \times 10^{7} \mathrm{~cm} / \mathrm{s}$, approximately $50 \mathrm{eV}$. Thus, the heating observed at fields greater than the highest (cold) resonance arises from warm ions which have leaked though the resonance. Other diagnostics confirm that these ions are traveling to the left; the reduction in $T_{i \perp}$ near the center of the device arise from cooling by the injected plasma. (Charge-exchange effects have not been included in the modeling.) 


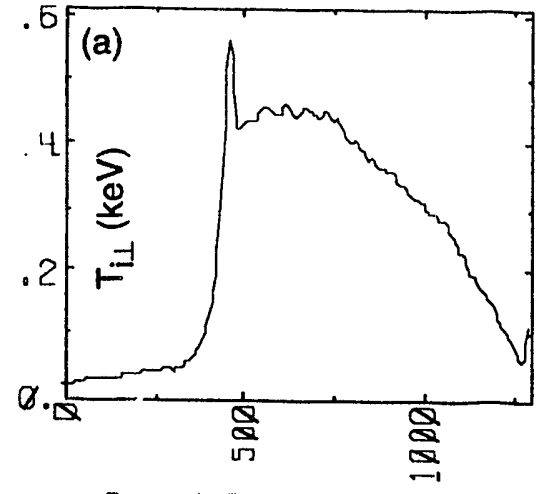

Axiol Position (cn)

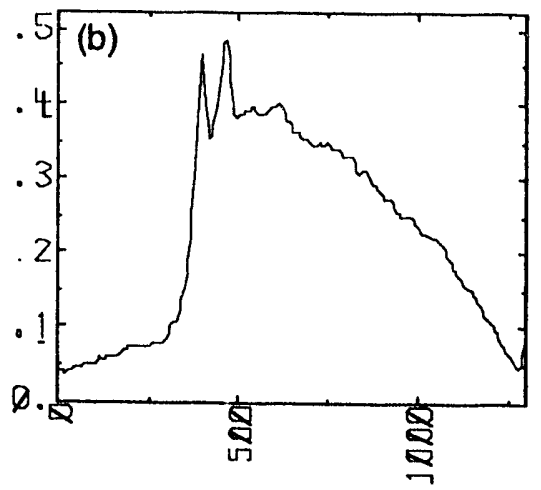

Axiol Position $\overline{\mathrm{cm}}$ )

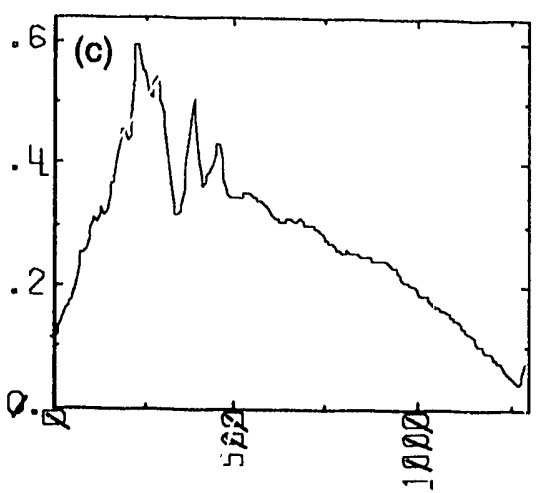

Axio| Pos ition (on)

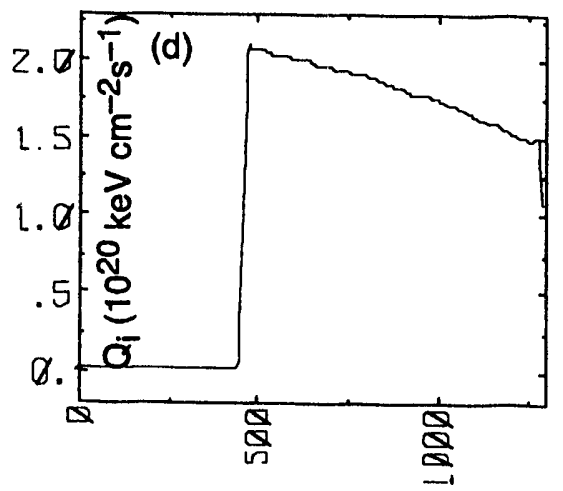

Axia I Pos ition $\overline{\mid c n}$

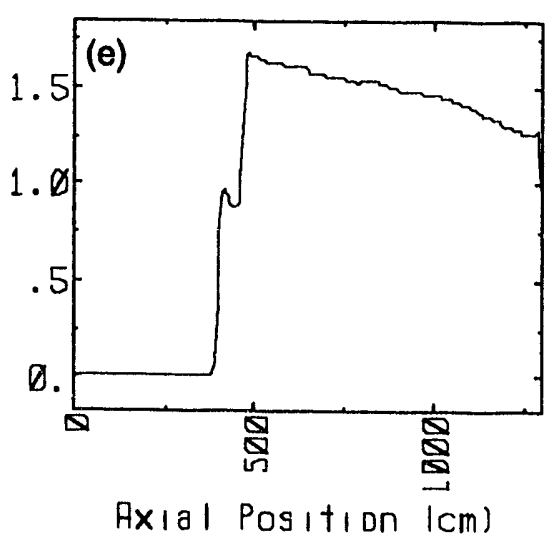

Axial Position $(\mathrm{cm})$

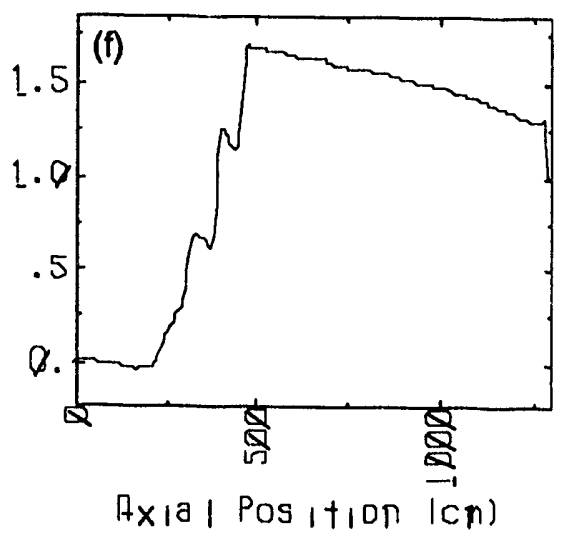

Figure 2.10. Heating at multiple ICRH frequencies. (a), (d) $\omega=2.44 \times 10^{8} \mathrm{~s}^{-1}, \mathrm{P}=56$ $\mathrm{kW} / \mathrm{cm}^{2}$;

(b), (e) $\omega=2.44 \times 10^{8} \mathrm{~s}^{-1}, \mathrm{P}=19 \mathrm{~kW} / \mathrm{cm}^{2}$ and $\omega=2.87 \times 10^{8} \mathrm{~s}^{-1}, \mathrm{P}=21 \mathrm{~kW} / \mathrm{cm}^{2}$;

(c), (f) $\omega=2.44 \times 10^{8} \mathrm{~s}^{-1}, \mathrm{P}=15 \mathrm{~kW} / \mathrm{cm}^{2} ; \omega=2.87 \times 10^{8} \mathrm{~s}^{-1}, \mathrm{P}=15 \mathrm{~kW} / \mathrm{cm}^{2}$; and $\omega=$ $3.31 \times 10^{8} \mathrm{~s}^{-1}, \mathrm{P}=15 \mathrm{~kW} / \mathrm{cm}^{2}$. Shown are the perpendicular ion temperature and ion heat flux. 

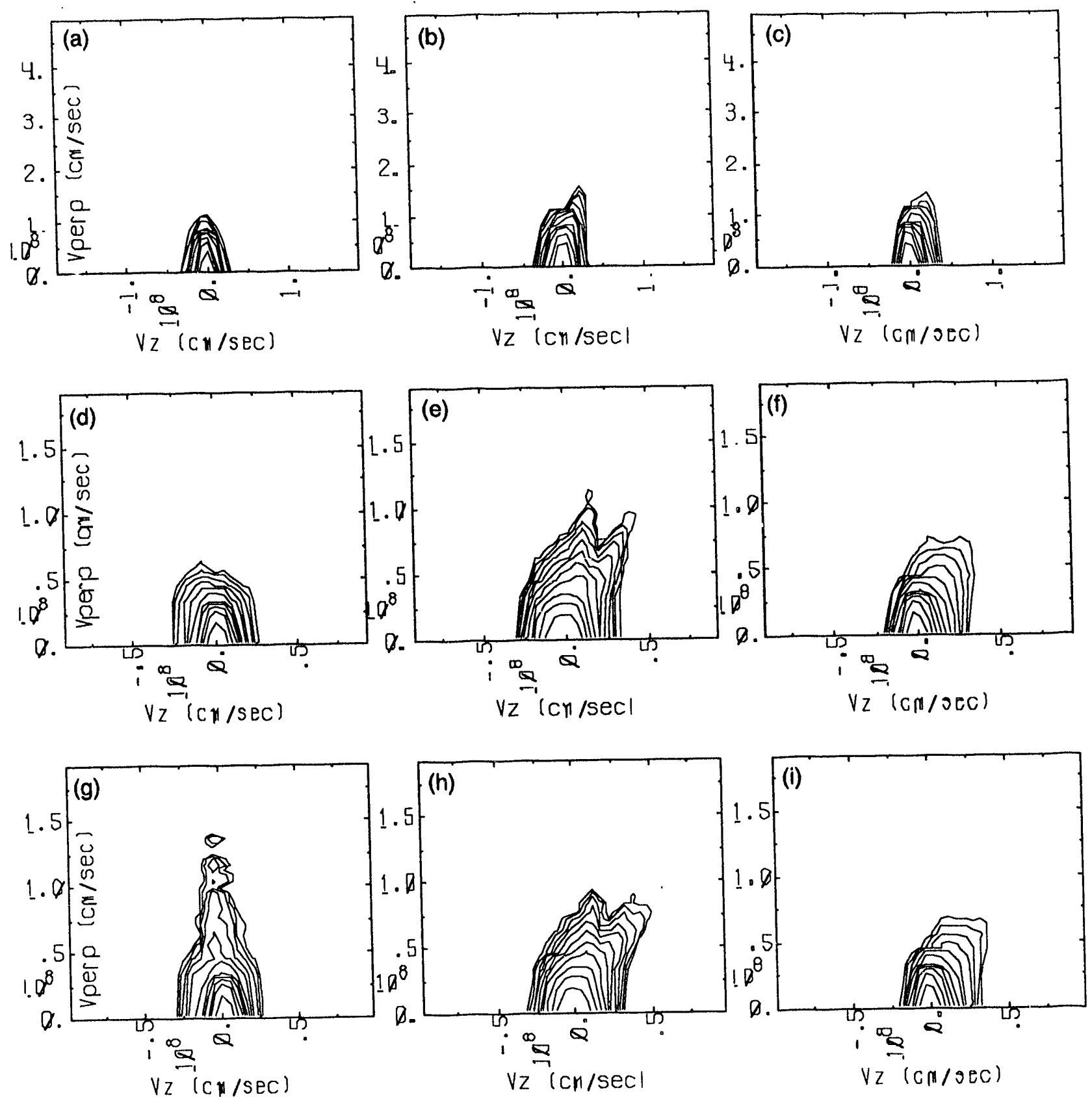

Figure 2.11. Velocity space contours. The three rows correspond to heating at 1,2, and 3 frequencies as in Fig. 2.10. The three columns are the three windows as in earlier figures. 


\subsection{Electron Heating by Landau Damping.}

The slow Alfvén wave has a parallel electric field arising from the finite electron mass. Although this component is normally neglected, it can generate significant electron heating through direct Landau damping. We consider this effect here.

As a first approximation to the simulator plasma, consider Alfvén waves propagating on a uniform column. In the limits of cold plasma and $m_{e}=0$, the plasma dispersion relation can be written: 1

$$
\left(1-\omega^{2} / \omega_{c i}^{2}\right) p_{z}^{4}-\left[2-p_{\perp}^{2}\left(1-\omega^{2} / \omega_{c i}^{2}\right)\right] p_{z}^{2}+\left(1-p_{\perp}^{2}\right)=0
$$

where $p=k v_{a} / \omega=n \omega_{p i} / \omega_{c i}$ and $v_{a}=\omega_{c i} c / \omega_{p i}$. The two solutions thus are

$p_{z}^{2}=\frac{1-p_{\perp}^{2}\left(1-\omega^{2} / \omega_{c i}^{2}\right) / 2 \pm \sqrt{\left[1-p_{\perp}^{2}\left(1-\omega^{2} / \omega_{c i}^{2}\right) / 2\right]^{2}-\left(1-\omega^{2} / \omega_{c i}^{2}\right)\left(1-p_{\perp}^{2}\right)}}{1-\omega^{2} / \omega_{c i}^{2}}$

The slow (shear or ion-cyclotron) wave has the + sign; note that in the limit $\omega^{2} / \omega_{c i}^{2} \rightarrow 1, p_{z}^{2}$ varies as $2 /\left(1-\omega^{2} / \omega_{c i}^{2}\right)$ for the slow wave. This formula, with the radial wave number $=3.83 /$ (plasma radius), yields wave vectors within about $30 \%$ of those determined for the non-uniform plasma. ${ }^{2}$ An approximation to Eq. (2) is included in the Landau damping part of the ICEPIC cade, so that the decrease in wave phase velocity near the resonance is modeled.

In the limit of zero Larmor radius, the wave polarizations are given in terms of the dielectric tensor components by the same expressions as in the cold plasma approximation:

$$
\begin{aligned}
& E_{x} \sim\left(\mathrm{K}_{\perp}-n^{2}\right)\left(\mathrm{K}_{\|}-n^{2} \sin ^{2}(\theta)\right) \\
& \sim\left(\mathrm{K}_{\perp}-n^{2}\right) \mathrm{K}_{\|} \\
& E_{y} \sim-\mathrm{K}_{x}\left(\mathrm{~K}_{\|}-n^{2} \sin ^{2}(\theta)\right) \\
& \sim-\mathrm{K}_{x} \mathrm{~K}_{\|} \\
& E_{z} \sim-\left(\mathrm{K}_{\perp}-n^{2}\right) n^{2} \sin (\theta) \cos (\theta)
\end{aligned}
$$

where the second forms for $E_{x}$ and $E_{y}$ hold in the limit of small electron mass. The electric field component along the magnetic field is nonzero only for finite $m_{e} / m_{i}$. From Eq. (1) we immediately find

1 W. P. Allis, S. J. Buchsbaum, and A. Bers, Waves in Anisotropic Plasmas, MIT Press, 1963.

2 R. W. Motley, et al., "ICRF Coil for the IDEAL Plasma," RF meeting, Boston (1993). 


$$
n^{2}=\frac{\omega_{p i}^{2}}{\omega_{c i}^{2}} \frac{1+p_{\perp}^{2}\left(1-\omega^{2} / \omega_{c i}^{2}\right) / 2+\sqrt{\left[1-p_{\perp}^{2}\left(1-\omega^{2} / \omega_{c i}^{2}\right) / 2\right]^{2}-\left(1-\omega^{2} / \omega_{c i}^{2}\right)\left(1-p_{\perp}^{2}\right)}}{1-\omega^{2} / \omega_{c i}^{2}}
$$

In the limit of small electron mass, we write

$$
\mathrm{K}_{\perp}=\frac{\omega_{p i}^{2}}{\omega_{c i}^{2}} \frac{1}{1-\omega^{2} / \omega_{c i}^{2}}
$$

and

$$
\mathrm{K}_{\times}=-i \frac{\omega_{p i}^{2}}{\omega_{c i}^{2}} \frac{\omega_{c i} / \omega}{1-\omega^{2} / \omega_{c i}^{2}}
$$

However, $K_{\|}$must include finite temperature effects, and is thus given by

$$
\mathrm{K}_{\|}=1+2 \frac{\omega_{p e}^{2}}{\omega^{2}} \frac{\omega^{2}}{k_{z}^{2} v_{T e}^{2}}\left[1+\frac{\omega}{k_{z} v_{T e}} Z\left(\frac{\omega}{k_{z} v_{T e}}\right)\right]
$$

with $v_{T e}=\sqrt{2 k T_{e} / m_{e}}$.

The magnitude of the field ratio is then given by

$$
\begin{aligned}
\left|\frac{E_{z}}{E_{\perp}}\right| & =\left(1+\left|\frac{\mathrm{K}_{\times}}{\mathrm{K}_{\perp}-n^{2}}\right|^{2}\right)^{-1 / 2}\left|\frac{n_{z} n_{\perp}}{\mathrm{K}_{\|}}\right| \\
& =\left(1+\mid \frac{\omega_{c i} / \omega}{p_{\perp}^{2}\left(1-\omega^{2} / \omega_{c i}^{2}\right) / 2+\sqrt{\left[1-p_{\perp}^{2}\left(1-\omega^{2} / \omega_{c i}^{2}\right) / 2\right]^{2}-\left(1-\omega^{2} / \omega_{c i}^{2}\right)\left(1-p_{\perp}^{2}\right)}}\right)^{2} \\
& \left|\frac{n_{z} n_{\perp}}{\mathrm{K}}\right|
\end{aligned}
$$

which as the wave approaches resonance becomes:

$$
\left|\frac{E_{z}}{E_{\perp}}\right| \sim\left|\frac{n_{z} n_{\perp}}{\sqrt{2} \mathrm{~K}_{\|}}\right|
$$

The plasma dispersion function is well approximated by

$$
Z(x) \sim i \pi^{1 / 2} e^{-x^{2}}-2 \frac{1}{\frac{1}{x}+\frac{1}{\frac{1}{2 x}+\frac{1}{4 x^{3}}}}
$$

where the expressions in the denominator yield the limits for $x 11$. Thus,

$$
\left|\mathrm{K}_{\|}\right|=\frac{\omega_{p e}^{2}}{\omega^{2}} 2 x^{2}\left[\pi x^{2} e^{-2 x^{2}}+\left(1-\frac{2 x^{2}\left(1+2 x^{2}\right)}{1+2 x^{2}+4 x^{4}}\right)^{2}\right]^{1 / 2}
$$


Consider the special case with $k_{z}=0.43, k_{\perp}=0.8 \mathrm{~cm}$ (both from Ref. 2), and

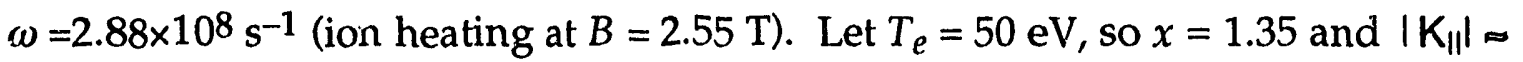
$0.56 \omega_{p e^{2}} / \omega^{2}$ in the approximation of Eq. (11). Thus, at $n=1 \times 10^{14} \mathrm{~cm}^{-3},\left|K_{\| 1}\right|=$ $3.0 \times 10^{6}$ and $E_{z} \approx 1.2 \times 10^{-3} E_{\perp}$. This is in good (order-of-magnitude) agreement with Ref. [2]; note, however, that they find a much larger $E_{z}$ near the plasma edge due to the structure of the mode.

For use in the code (c.f. Appendix 2.A), we also need $\Delta k_{z}$; from Ref. [2] we estimate a half-width in the $k_{z}$ spectrum of $0.06 \mathrm{~cm}^{-1}$. Note, however, that the damping is not sensitive to this value as long as it is small compared with $k_{z}$.

A series of calculations were performed using the true electron mass, so as to properly calculate the filling of the quasilinear plateau resulting from the damping. The starting point was an ICRH-only case with $S=6.64 \times 10^{19}$ $\mathrm{cm}^{-2} \mathrm{~s}^{-1}$ and $P_{\text {ICRH }} \sim 50 \mathrm{~kW} / \mathrm{cm}^{2}$. The nominal parallel electric field of 1.56 $\mathrm{V} / \mathrm{cm}$ corresponded to an additional $17 \mathrm{~kW} / \mathrm{cm}^{2}$ of electron heating; the field was varied above and below this value with the results shown in Fig. 2.12. We see that an electron temperature of $100 \mathrm{eV}$ can be obtainerd with direct electron heating of about $55 \mathrm{~kW} / \mathrm{cm}^{2}$. For a nominal column radius of $5 \mathrm{~cm}$ and a peaking factor of 2 in the rf power, this corresponds to approximately 2 MW of power in ICRH ion heating and $2 \mathrm{MW}$ in direct electron heating per end of the device. (A choke coil could make the losses nearly single ended.)

The heating of the electrons from ion collisions or other direct heating mechanisms is needed to raise $T_{e}$ sufficiently that the thermal electron velocity is comparable to the wave phase velocity. In the absence of this heating, the Landau damping will be weak and the electron temperature not enhanced significantly. As a result, Landau damping by the relatively large $E_{\|}$ near the plasma edge requires a self-consistent model of the plasma heating by other mechanisms; if these effects are weak, the damping will also be weak. If, on the other hand, these effects can raise $T_{e}$ to about $30 \mathrm{eV}$, then heating by Landau damping near the edge may play a major role both in the local power balance and in limiting the wave power which can reach the plasma center to heat both ions and electrons there.

The equilibrium which results from Landau damping at $P_{L D}=53$ $\mathrm{kW} / \mathrm{cm}^{2}\left(E_{L D}=3.12 \mathrm{~V} / \mathrm{cm}\right)$ is shown in Fig. 2.13. Note from the ion heat flux plot that less than $25 \%$ of the ion heating is transferred to the electrons. The density is reduced from that in the absence of Landau damping, and the potential drop to the end significantly increased to confine the heated electrons. 


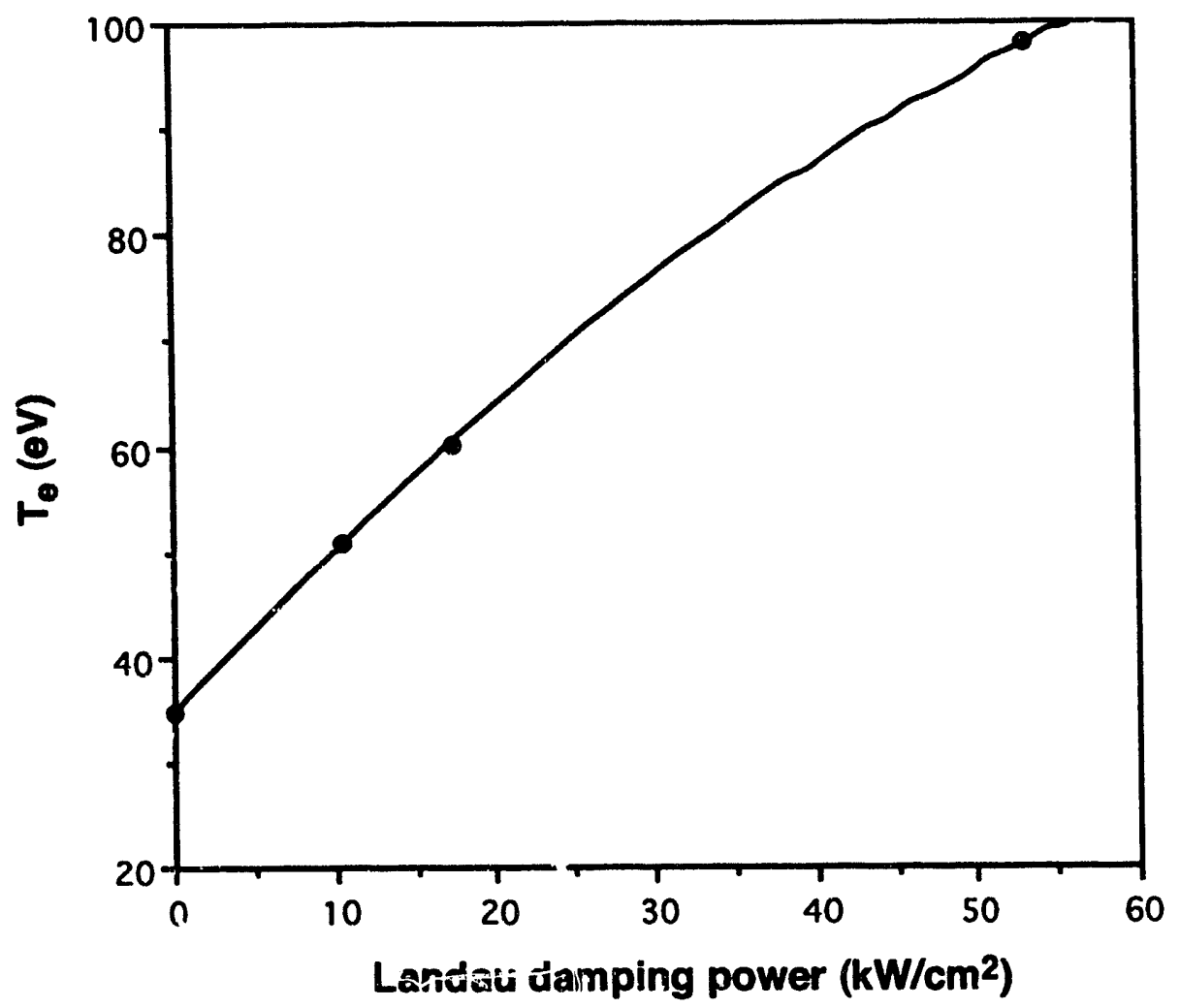

Figure 2.12. Flectron temperature vs. direct electron heating. See text for discussion and parameters. 

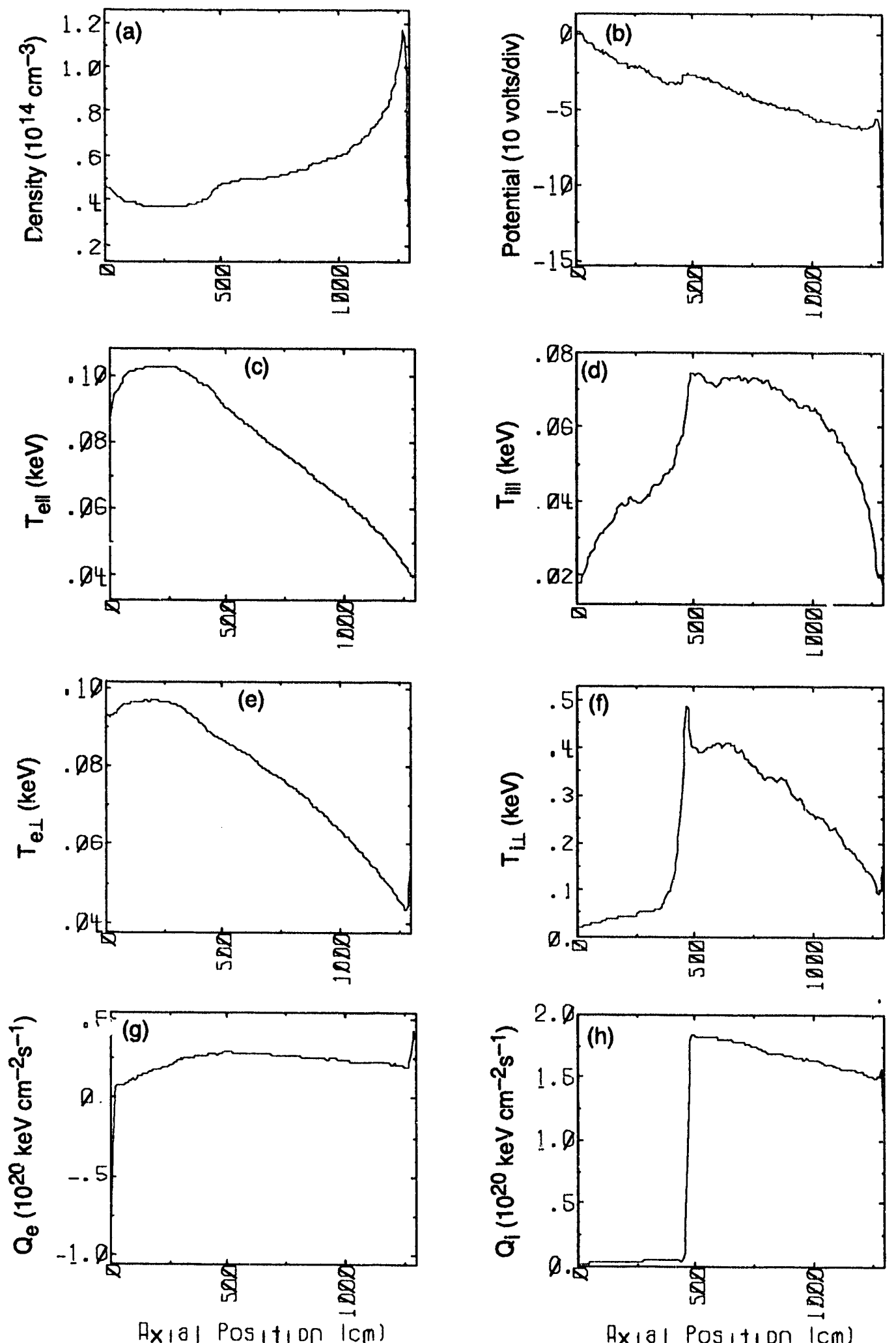

Figure 2.13. Equilibrium for $P_{I C R H}=44.8 \mathrm{~kW} / \mathrm{cm}^{2}, P_{L D}=53.3 \mathrm{~kW} / \mathrm{cm}^{2}, S$ $=6.64 \times 10^{19} \mathrm{~cm}^{-2} \mathrm{~s}^{-1}$. True electron mass. 

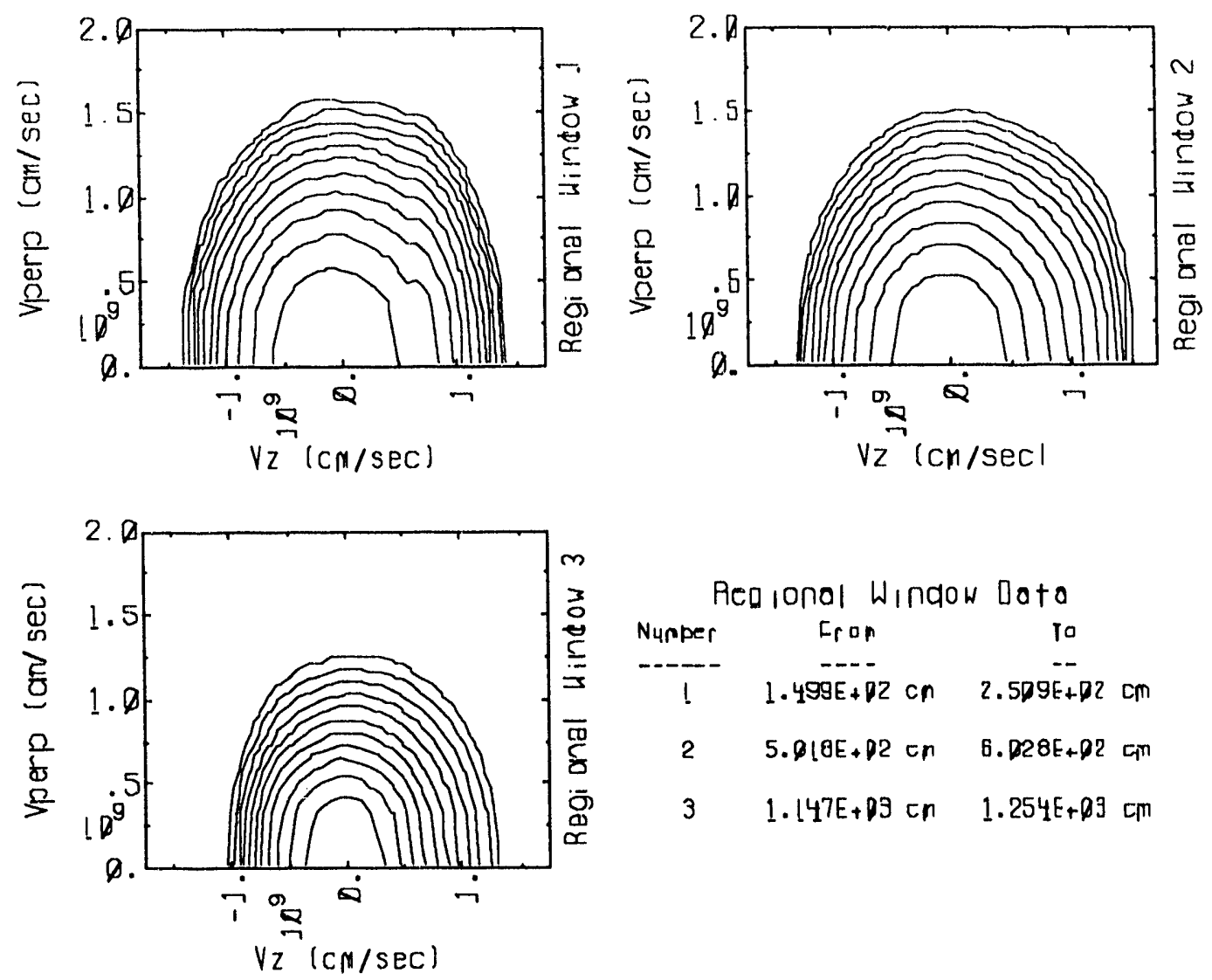

Fig ure 2.14. Electron velocity contours for the same case as Fig. 2.13. 

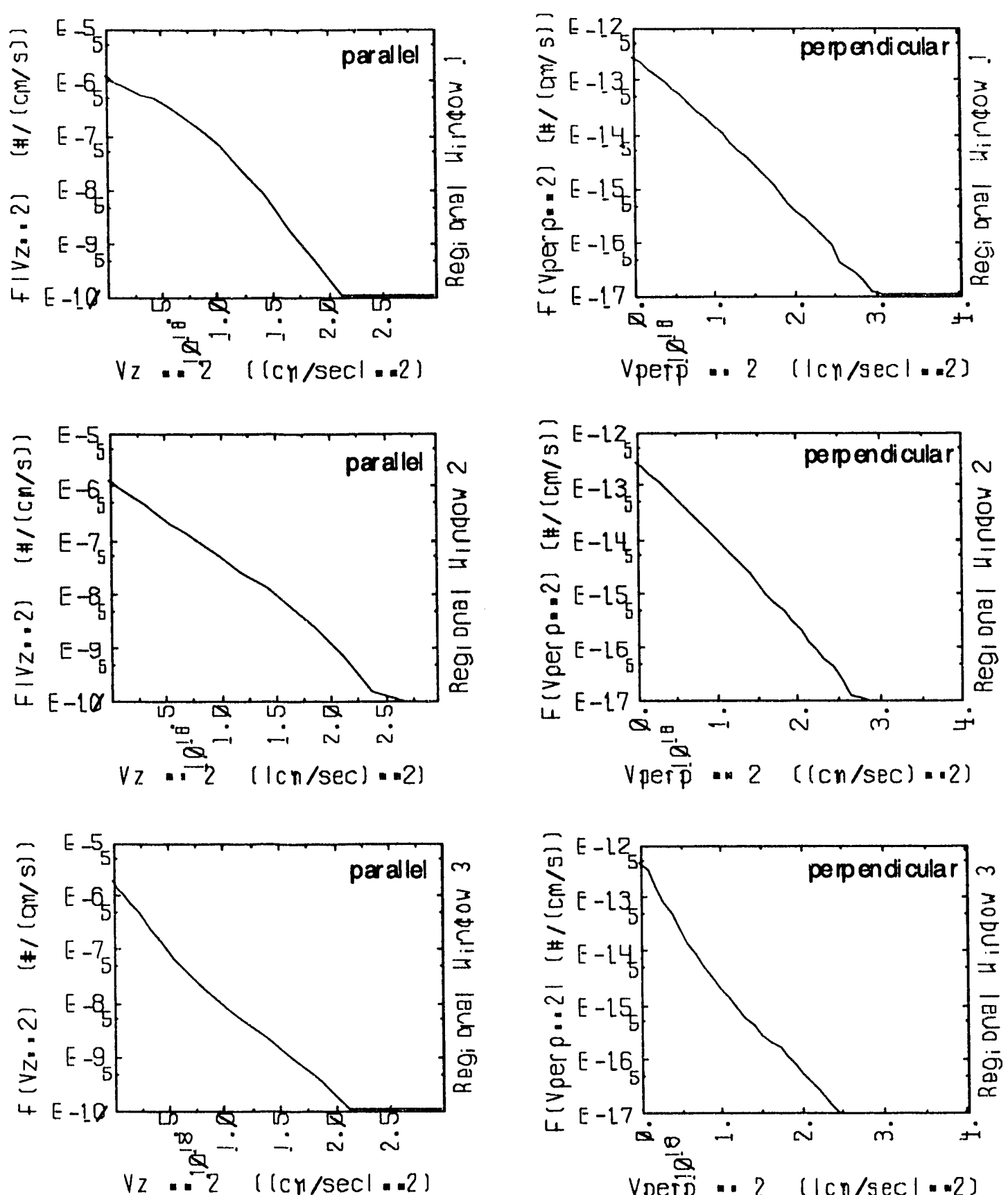

Figure 2.15. Electron distribution fundion for the same case as Figs. 2.13, 2.14. 


\subsection{Electron Cyclotron Resonance Heating.}

The electron heating required to reach $T_{e} \sim 100 \mathrm{eV}$ can be provided by electron cyclotron resonance heating (ECRH). We have modeled this for the particular case that the electron and ion resonances are at the same location, $B$ $=2.55 \mathrm{~T}$, close to the uniform, downstream value of $2.5 \mathrm{~T}$ so as to minimize the effects of the $\mu \nabla B$ forces. The results are shown in Figs.2.16-2.17 (true electron mass), for $S=1.66 \times 10^{20} \mathrm{~cm}^{-2} \mathrm{~s}^{-1}, P_{\text {ICRH }}=49 \mathrm{~kW} / \mathrm{cm}^{-2}$, and $P_{\text {ECRH }}=$ $62 \mathrm{~kW} \mathrm{~cm}^{-2}$. Densities are similar to previous cases. The electron distribution is ansiotropic near the resonance, but quickly thermalizes as can be seen from both the electron temperature plots and the distribution function contours of Fig. 2.17. Note from the heat flux plots, Fig. 2.17, that the electron and ion distributions are only weakly coupled for this case; at higher fueling rates we anticipate a stronger coupling.
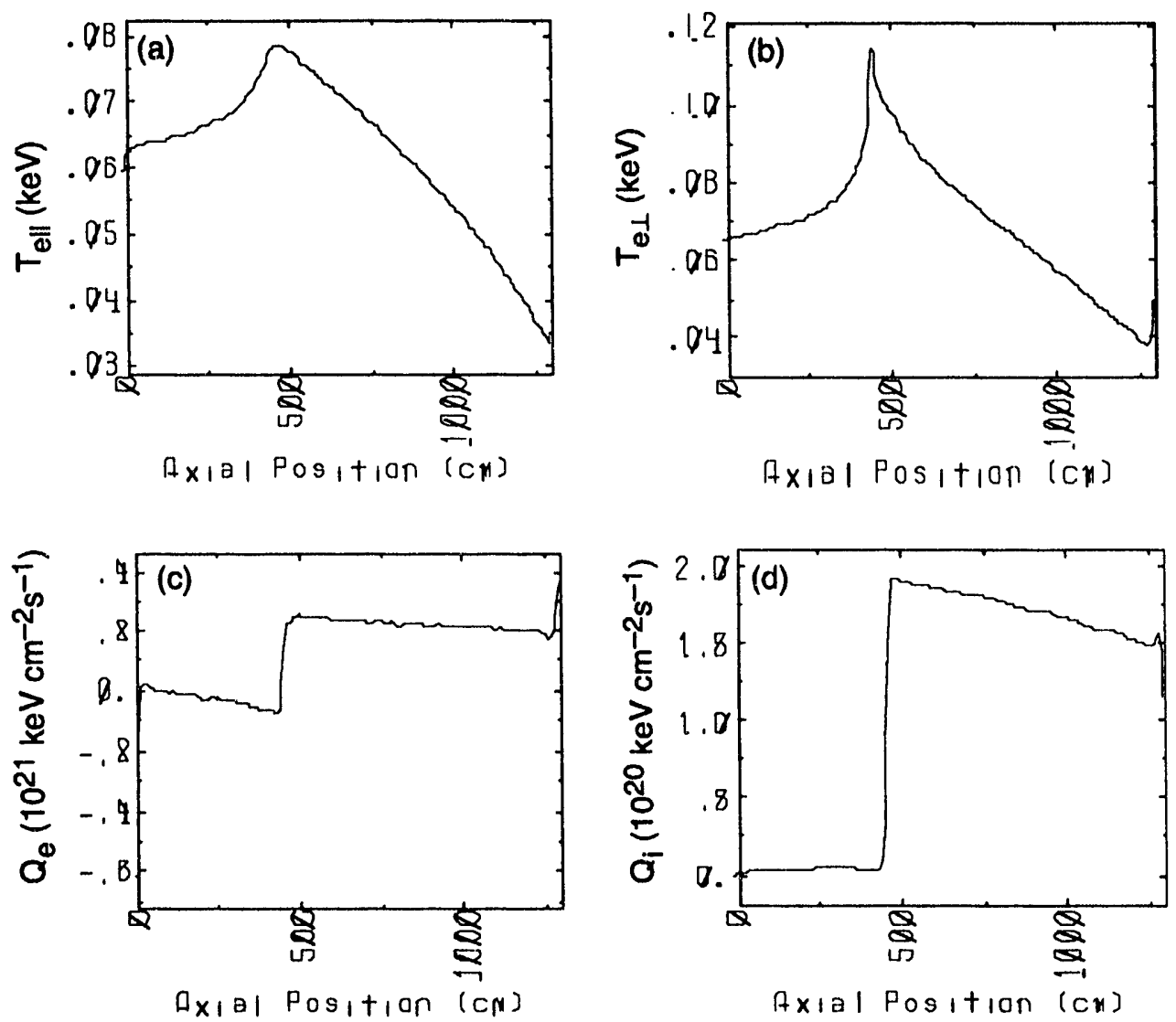

Figure 2.16. Results of ECRH. (a)-(b) Parallel and perpendicular electron temperatures. (c)-(d) Electron and ion heat fluxes. 

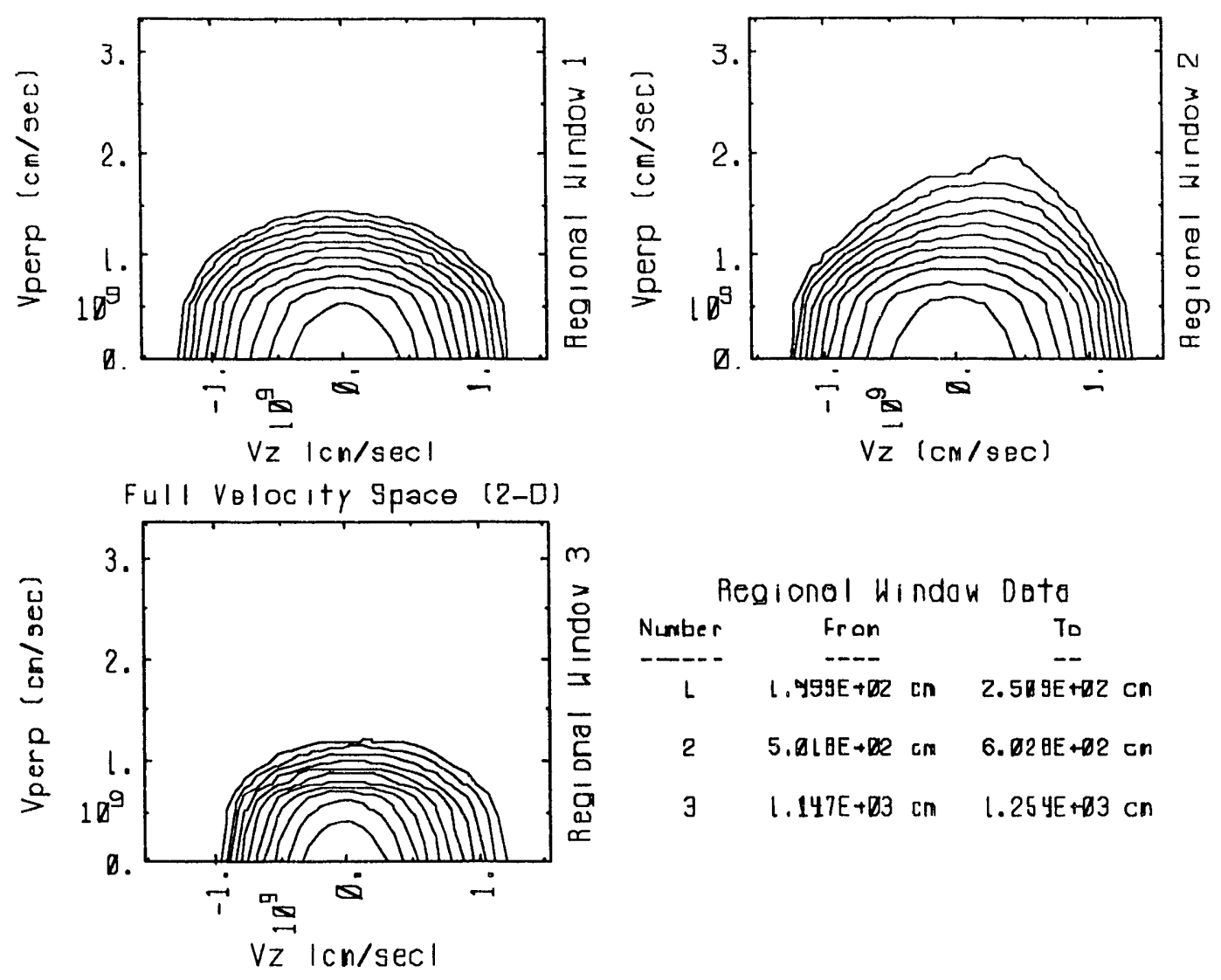

Figure 2.17. Electron distributions during ECRH. (Case dv39.)

\subsection{Direct Radial Transport due to ICRF.}

The resonant heating of ions at the cyclotron resonance produces a change in the ion magnetic moment and energy. From the Hamiltonian structure and the resonance condition, the heating characteristics for any cyclotron wave are straightforwardly deduced. ${ }^{3}$ From the heating characteristics, it is easily shown that a cyclotron-resonant wave produces a concomitant shift in the resonant species guiding centers accompanying the changes in the magnetic moments and energies. The guiding center shift is

$\Delta \mathrm{Y}=-\left(\Delta \mathrm{E} / \mathrm{mc}^{2}\right)\left(\mathrm{c} / \Omega_{0}\right) \mathrm{N}=-\mathrm{O}(1)\left(\Delta \mathrm{E} / \mathrm{mc}^{2}\right)^{1 / 2} \mathrm{~N} \rho$

where $\Delta \mathrm{E}$ is the energy change, $\mathrm{N}=\mathrm{k} c / \omega$, and $\rho$ is the Larmor radius after heating. For the ICRF parameters proposed for IDEAL, $|\Delta Y / \rho|<<1$.

3 B. I. Cohen, R. H. Cohen, T. D. Rognlien, and W. M. Nevins, Rev. Mod. Phys. 63, 957 (1991). 


\subsection{ICRF Pondercmotive Effects.}

A number of experiments and theoretical studies have established that ion cyclotron waves produce a ponderomotive potential that can aid in both confining plasma and stabilizing magnetohydrodynamic (MHD) instability. Some of the most relevant experimental work on ICRF ponderomotive stabilization of MHD modes has come from the Phaedrus tandem mirror at the University of Wisconsin. The ponderomotive potential, ${ }^{4}$

$$
\psi_{p}=e^{2} E_{z}{ }^{2} / 4 m \omega+\left(e^{2} / 4 m \omega\right)\left[E_{L}^{2} /(\omega-\Omega)+E_{R}^{2} /(\omega+\Omega)\right],
$$

where $\Omega \approx e B / m c$ (positive for ions and negative for electrons), is stabilizing for left-circularly polarized ICRF with $\omega<\Omega$ and $E_{L}{ }^{2}$ peaked in the center of the plasma, as will be the case for the slow waves in IDEAL. For the power levels proposed in IDEAL, ponderomotive effects will be significant.

\subsection{Parametric Instability.}

Parametric instabilities associated with ion cyclotron heating have been examined much more extensively for ICRF frequencies above the ion cyclotron frequency. 5 For wave frequencies less than the ion cyclotron as proposed in IDEAL, the possible decay channels are limited by the relative lack of normal modes in this frequency range. Porkolab has suggested that the slow wave is likely to be parametrically unstable to decay into a type of Trivelpiece-Gould mode with dispersion relation

$$
1-\left(\mathrm{k}_{\perp}{ }^{2} / \mathrm{k}^{2}\right)\left(\omega_{\mathrm{pi}^{2}}{ }^{2} /\left(\omega^{2}-\Omega_{\mathrm{i}}{ }^{2}\right)-\left(\mathrm{k}_{\|^{2}} / \mathrm{k}^{2}\right) \omega_{\mathrm{pe}^{2}} / \omega^{2}=0\right.
$$

and a quasi-mode. Porkolab expects this to be of partirular interest near the edge of the plasma and might result in enhanced elec'ron heating. For a plasma with two ion species there are additional possibilities for parametric decay and the ion-ion hybrid resonance contributes. ${ }^{6}$ Analysis is generally useful for assessing whether parametric instabilities are likely to occur, but one cannot predict with any confidence how much of the ICRF wave power will be expended in parametric instabilities. However, the experimental experience with ICRF in TMX, Phaedrus, and other experiments is that parametric instabilities may contribute to the absorption but do not prevent the ICRF from coupling well to the plasma and doing substantial heating.

4 G. Dimonte, B. M. Lamb, and G. J. Morales, Phys. Rev. Lett. 48, 1352 (1982).

5 M. Porkolab, Fusion Engr. and Design 12, 93 (1990).

${ }^{6}$ M. Ono, Phys. Rev. Lett. 42, 1267 (1979). 


\section{A ICEPIC Simulations of RF Heating and Collisional Coupling}

ICEPIC is a self-consistent, electrostatic, particle-in-cell simulation code. It was developed by Richard Procassini from an earlier particle code authored by Procassini and Cohen, who applied it to the study of magnetic-mirror confined plasistas and divertor physics. ${ }^{78}$

ICEPIC follows the motion of electrons (possibly relativistic) and ions in one spatial dimension parallel to the magnetic field (possibly inhomogeneous) and in two velocity dimensions $\left(v_{\|}, v_{\perp}\right)$. The parallel electric field used to accelerate the particles is determined self-consistently by a direct-implict solution coupling Poisson's equation and the particle equations of motion to allow large time steps, i.e., $\omega_{\text {pe }} \Delta t>>1 .{ }^{9}$ The $\mu \nabla B$ parallel force is included for both species. The accuracy constraint on the simulation time step is that the electron transit time across the shortest interesting wavelength retained must exceed the time step. When $\omega_{\text {pe }} \Delta t>1$, the spatial grid dimension must exceed the Debye length which results in a loss of resolution of the Debye sheath near a conducting plate; and the potential drop through the sheath is only approximately correct.

A binary collision model for Coulomb collisions is used for both species patterned after the method of Takizuka and Abe. ${ }^{10}$ The algorithm conserves momentum and energy, and makes no assumption that the distribution functions remain nearly Maxwellian. The collision model is fully equivalent to a complete self-consistert solution of the time-dependent Fokker-Planck equations for the ion and electron distribution functions incorporating electron-electron, ion-ion, and electron-ion collisions.

ICEPIC includes distributed or point particle sources to model ionization of gas or recycling on a surface. There is also a charge-exchange model. There are several models of wave heating available in the code.11 The code uses a quasilinear Fokker-Planck model for ion and electron cyclotron heating based on the formalism of Bernstein and Baxter ${ }^{12}$ and the Monte Carlo implementation scheme of Rognlien. ${ }^{13}$ The decorrelation mechanism for the quasilinear cyclotron resonant heating is the spatial inhomogeneity of the magnetic field through the cyclotron resonance. The parallel electric-field

\footnotetext{
7 R. J. Procassini, C. K. Birdsall, and B. I. Cohen, Nucl. Fusion 30, 40 (1990).

8 R. J. Procassini and B. I. Cohen, J. Comput. Phys. 102, 39 (1992).

${ }^{9}$ B. I. Cohen, A. B. Langdon, and A. Friedman, J. Comput. Phys. 46, 15 (1982).

10 T. Takizuka and H. Abe, J. Comput. Phys. 25, 205 (1977).

${ }_{11}$ R. J. Procassini and B. I. Cohen, J. Comput. Phys. 102, 39 (1992).

12 I. B. Bernstein and D. C. Baxter, Phys. Fluids 24, 108 (1981).

13 T. D. Rognlien, Nucl. Fusion 23, 163 (1983).
} 
component of a wave used for heating can heat either species by the mechanism of Landau resonance. ICEPIC now includes a quasilinear FokkerPlanck model of heating due to Landau damping of the parallel electric field component of an imposed wave in ICEPIC with a parallel velocity diffusion coefficient:

$$
D_{v v}=<\Delta v^{2} / 2 \Delta t>=(1 / 2)\left(e E_{\|} / m\right)^{2} v /\left[\left(\omega-k_{\|} v_{\|}\right)^{2}+v^{2}\right]
$$

where the decorrelation frequency $v=\left(\omega / k_{\|}\right) \Delta k$ is assumed to be caused by a spread in wavenumbers produced by the wave launching structure. For $|v / \omega|<<1$, this diffusion coefficient gives a heating rate equal to the Landau damping rate. To exercise the wave heating options in the simulation, the amplitudes of the wave fields used for heating must be specified. Thus, ICEPIC has the flexibility to study wave heating of the electrons and ions due to cyclotron or Landau resonance, the collisional coupling of the species, and the self-consistent flow of plasma parallel to an imposed nonuniform magnetic field. 
Appendix 2.B - Comparison of quasilinear heating model with detailed equation of motion for strong $\mathbf{R F}$

2.B.1 Description of the models. The magnitude of the RF electric field for the proposed ion cyclotron resonance heating is sufficiently large that an ion can be heated to a number of $\mathrm{keV}$ in one pass through the resonance zone. The quasilinear model that is used in the ICEPIC simulations is derived on the assumption that the change in ion energy is only a small perturbation. Furthermore, if the change in energy is negative and sufficiently large, one many obtain a negative energy from the quasilinear model that must be corrected somehow. In this section, we compare the quasilinear model in ICEPIC with a more accurate numerical solution to the equations of motion to verify the adequacy of the ICEPIC model even though the change in ion energy can be very large.

To check the quasilinear model in ICEPIC, we use a code call ORPAT that solves the particle equations of motion through the cyclotron resonance including the gyrophase, as described in Ref. [1]. ${ }^{1}$ This code also has two quasilinear models that can be used for direct comparison; one model does a multi-step quasilinear diffusion process for an ion that has had a cyclotron resonance in such a way that each of the steps results in a small change in the ion energy, and the second model does the diffusion in one step, as in ICEPIC. The quasilinear model gives the change in energy in the form ${ }^{2}$

$$
\frac{m}{2} v^{2} \rightarrow \frac{m}{2} v^{2}+a v E^{2}+b E R_{n}
$$

where $v$ is the (perpendicular) velocity, $E$ is the electric field strength, $a$ and $b$ are constants depending on the particle velocity and gradient of the magnetic field, and $R_{n}$ is a random number uniformly distributed between -1 and 1 . For the one-step model, the absolute value of the right-hand side is used for cases where the new $v^{2}$ is negative. This can be thought of as having a reflecting boundary at $v=0$, although it is not rigorously justified and probably works fairly well only for heating at the fundamental cyclotron resonance, but not at harmonics.

2.B.2 Calculations for divertor simulator. The comparison is performed on the magnetic field profile used by ICEPIC for a cyclotron resonance midway down the beach at $B_{\text {res }}=3 \mathrm{~T}$. A finite parallel index of refraction of $N_{\|}=90$ is assumed, which splits the heating zones spatially because of the Doppler shift; ions with negative parallel velocities are resonant at a higher magnetic field

1 T.D. Rognlien, Phys. Fluids, Vol. 26, 1545 (1983).

2 See, for example, Y. Matsuda and T.D. Rognlien, Nucl. Fusion, Vol. 21, 347 (1981). 
and positive parallel velocities are resonant below the $3 \mathrm{~T}$ point. For a RF uniform electric field, we use a magnitude of $40 \mathrm{~V} / \mathrm{cm}$ which gives several MW of absorbed power for a density of $5 \times 10^{19} \mathrm{~m}^{-3}$ and an area of $50 \mathrm{~cm}^{2}$. Each time an ion reaches the right or left boundary, it is replaced by an ion chosen randomly from a $50 \mathrm{eV}$ Maxwellian, and reinjected. Because of the magnetic hill in the center and the $\mu \nabla B$ force on the heated ions, roughly 9 out of 10 ions leave at the right-hand end wall boundary. There is no electrostatic potential for these calculations.

The ion distributions at the end wall for the three models are shown in Fig. 2B.1. Note the strong similarity and the fact that all three show an ion tail extending to about $10 \mathrm{keV}$, although the mean energy is about $300 \mathrm{eV}$. These distributions are very comparable to what is found in the ICEPIC calculations. The location of the heated ions in the $\left(v_{\perp}, v_{\|}\right)$space corresponds to the so-called heating characteristic when viewed from the end wall where $B=(2.5 / 3.0) B_{\text {res. }}$.

To be more quantitative, we compare the energy absorbed for the three models. These absorption profiles are given in Fig. 2B.2, where we only show a $3 \mathrm{~m}$ region near the resonance and $B=3 \mathrm{~T}$ at $z=0 \mathrm{~cm}$. Also shown are the integral of the absorption profiles to give the total power for a density of $5 \times 10^{19} \mathrm{~m}^{-3}$. Because of the Doppler shift, the absorption to the left of $z=0$ is due to ions with negative parallel velocity. Note that all the models show the absorption divided into two lobes about $z=0$, although the detailed equations of motion show a broader profile. The total power absorbed in the three cases is as follows:

$$
\begin{array}{ll}
\text { equations of motion } & 4.3 \times 10^{23} \mathrm{eV} / \mathrm{cm}^{2} \\
\text { multi-step quasilinear } & 3.5 \times 10^{23} \mathrm{eV} / \mathrm{cm}^{2} \\
\text { single-step quasilinear } & 6.2 \times 10^{23} \mathrm{eV} / \mathrm{cm}^{2}
\end{array}
$$

The data for the equations of motion has been averaged over four runs (other three not shown) because each such run used about a factor of 4 few particles than the quasilinear runs owing to the equations of motion calculations being more expensive. Even though the single-step quasilinenr overestimates the heating by roughly $50 \%$, it is surprisingly accurate given the magnitude of the step size. We attribute this to the weak velocity dependence of the fundamental frequency heating, and would not expect it to hold for the second harmonic heating, for example. We view the comparison of the three models as a justification for using the single-step quasilinear model; its basic shape of the heated distribution is correct, and even if the power absorbed is somewhat inflated, one can simply reduce the electric field to compensate for it as we do not have an accurate way to determine the electric field anyway. 
Finally, we note that we have tried some variations with half the $N_{11}(=45)$ and with heating limited to the high magnetic field side $\left(\mathrm{v}_{\|}<0\right.$ ions). The basic correspondence between the models given above still holds. 

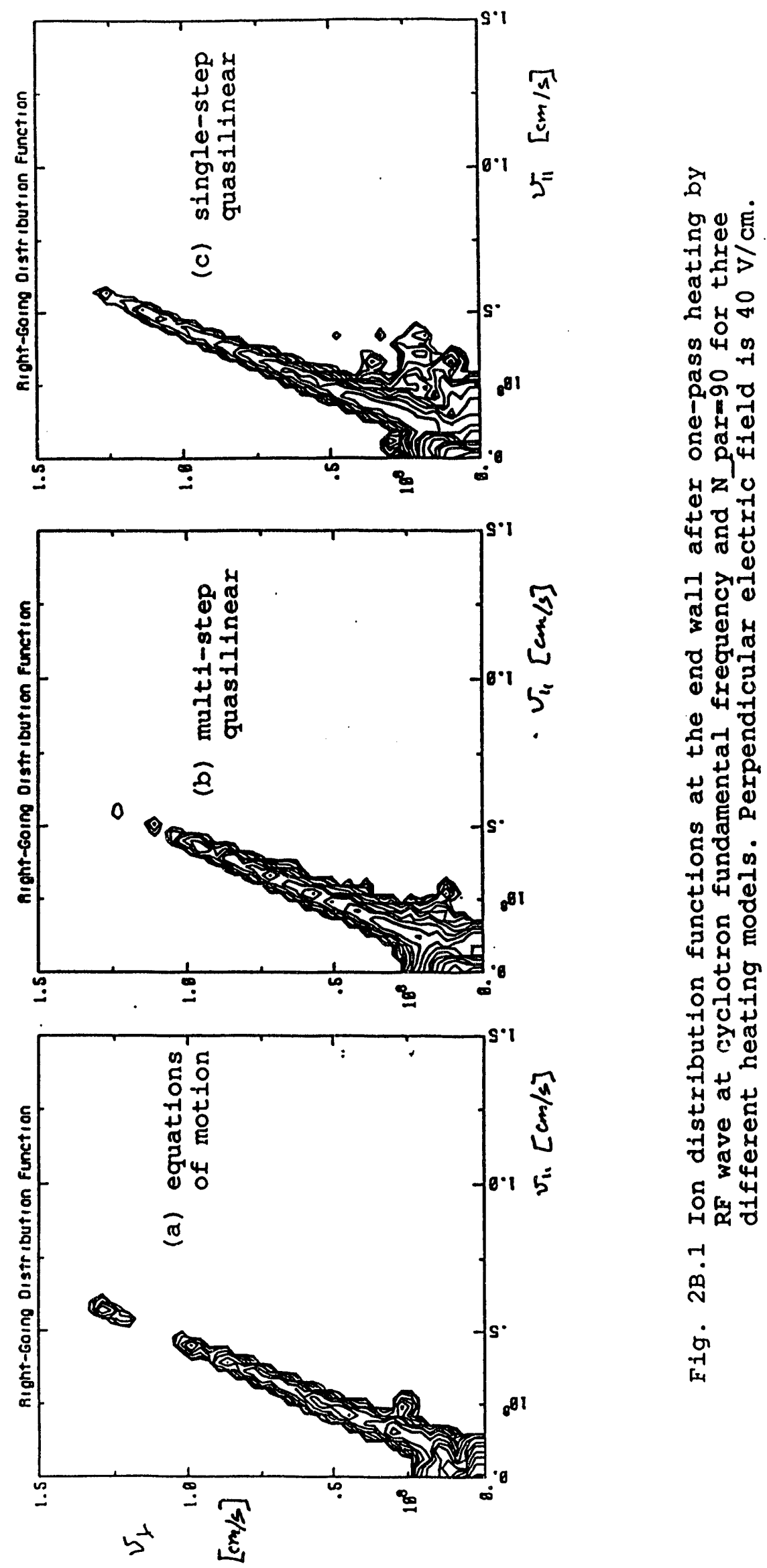

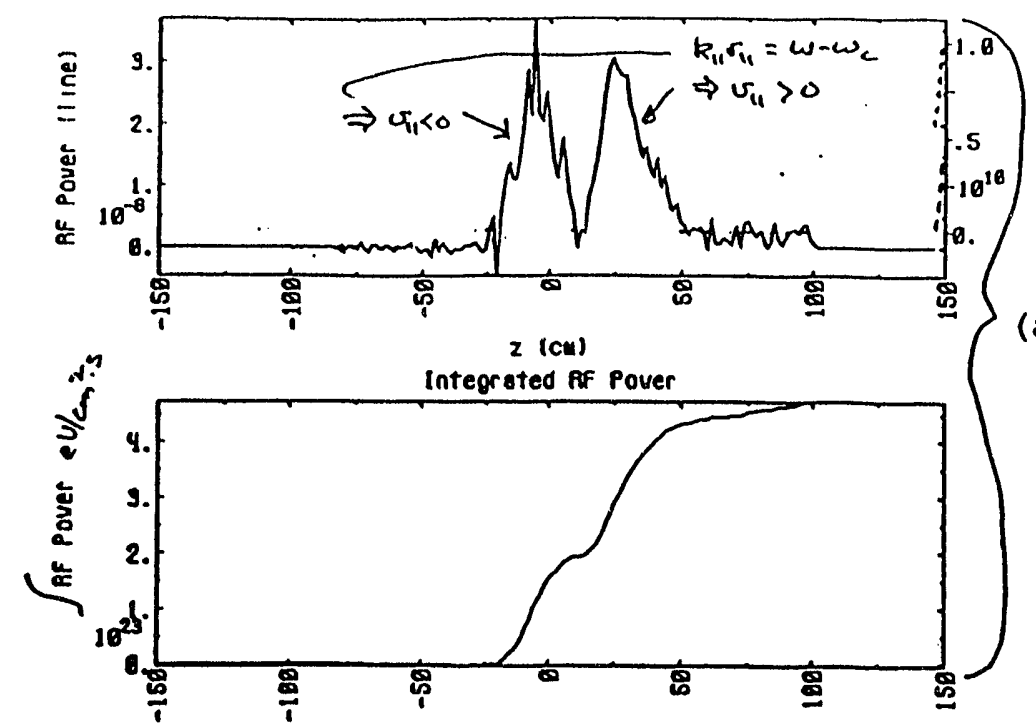

(a) equations of motion
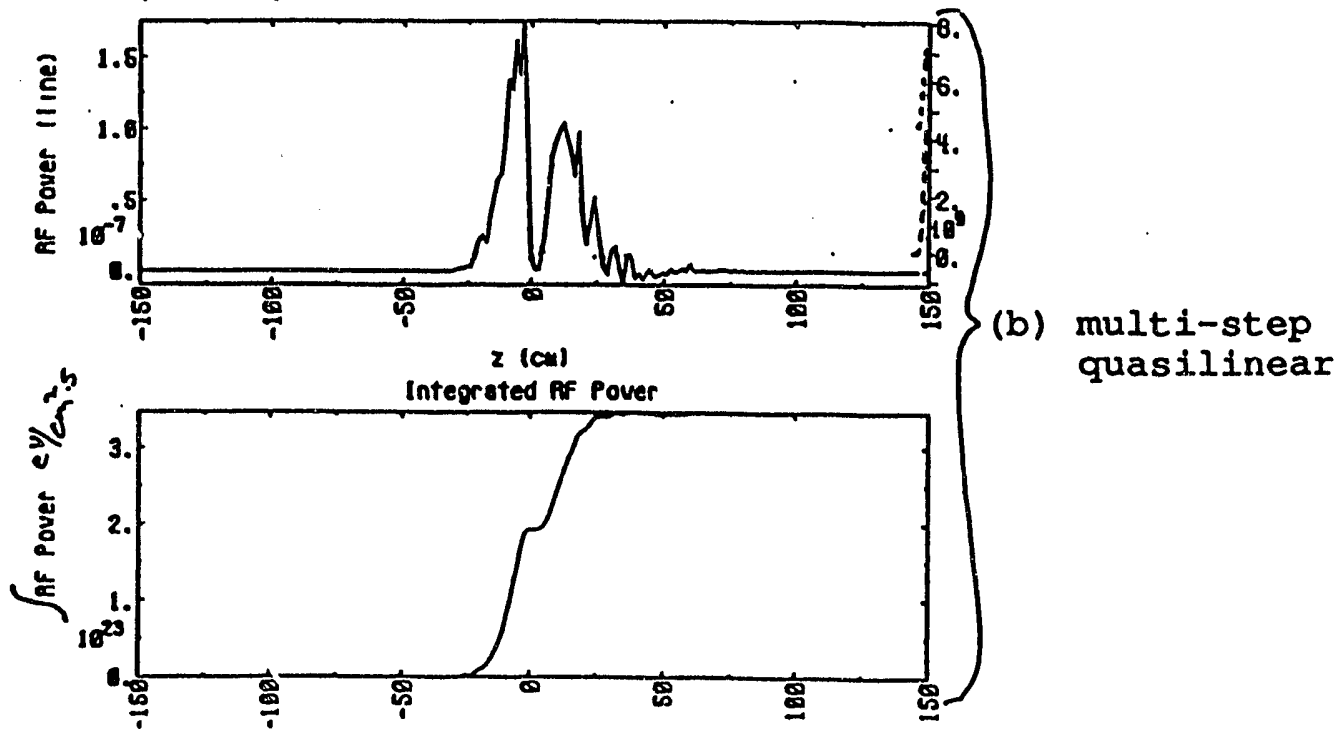

quasilinear
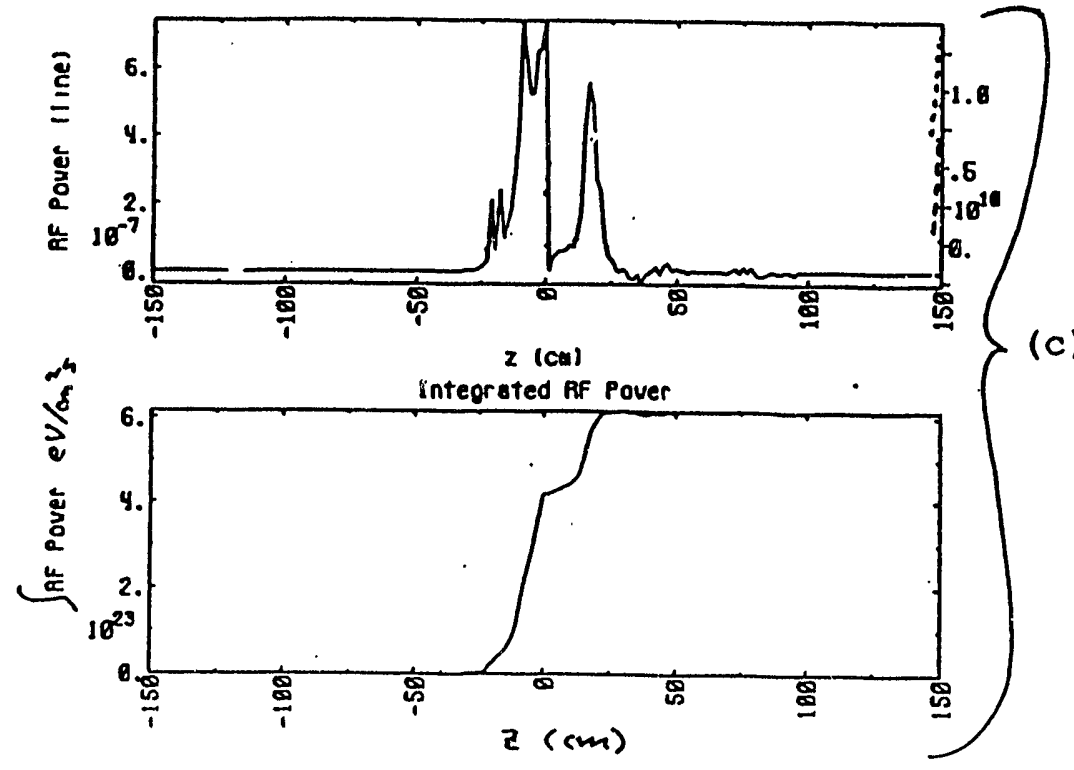

(c) single-step quasilinear

Fig. 2B.2 Power absorption profiles for the three heating models for the parameters in Fig. 2B.1. Also shown is the integrated power. 


\title{
3. ICRF Heating of Electrons in IDEAL:
}

\section{Mode Conversion and Landau Danping at the Alfvén resonance}

\author{
J.A. Byers
}

3.1 Introduction. The following is a brief review of the literature regarding the use of ICRF for electron heating. The calculations required to quantify the effect are determined but not carried out here. The most discussed process for $\omega \leq \omega_{c i}$, which is the planned frequency range for excitation of slow modes in IDEAL, is a two step process whereby the plasma mode excited by the antenna propagates inward to the Alfvén resonance in the radially varying density, where it becomes singular in the cold plasma limit, or in the full thermal limit mode-converts to the kinetic Alfvén wave (KAW), which then propagates further inwards and rapidly damps via electron Landau damping. The slow mode excited by the antenna itself has weak Landau damping even if $\omega / k_{z} v_{t e} \approx 1$ because $E_{\|}$is very small. In coninst, the mode it converts to, the kinetic Alfvén wave has high $k_{\perp}$ and appreciable magnitude of $E_{\|}$.

This physical process is distinct from the ion heating at a magnetic beach, which is the primary present focus of the Ideal model. The mode conversion into the KAW in the Alfvén continuum and subsequent Landau damping is capable of directly heating the electrons (or the ions), and would likely take place directly in the axial vicinity of the antenna, before any significant propagation to the beach region occurs. It should also continue to operate as the wave propagates axially away from the antenna, and would find its maximum heating at an axial location away from the antenna where $k_{z}$ has increased enough so that $\omega / k_{z} v_{i_{-}} \therefore 1$. Directly underneath the antenna, $k_{z}$ is determined by the antenna structure $k_{z} \approx 0.4 \mathrm{~cm}^{-1}$, so

$$
\omega / k_{z} v_{t e} \approx 1.5 \rightarrow 2.2
$$

for

$$
\omega=\omega_{c i}(z)=2.510^{8} \sec ^{-1}(B=2.6 T) \rightarrow 2.8710^{8} \sec ^{-1}(B=3.0 T)
$$

and for

$$
v_{t e}=3.2510^{8} \mathrm{~cm} / \mathrm{s}\left(T_{e}=30 \mathrm{ev}\right) \rightarrow 4.2010^{8} \mathrm{~cm} / \mathrm{s}\left(T_{e}=50 \mathrm{ev}\right)
$$

For these parameters the mode must increase its $k_{z}$ by only a factor of 2 by propagating axially into the decreasing $B(z)$ to obtain optimum electron Landau damping.

Most of the discussion in the literature follows ideas developed in Hasegawa and Chen[1], whereby the Alfvén continuum in a continuously variabie density profile phase mixes and provides damping in the cold plasma model. In the kinetic model HC show that the mode converts to a kinetic Alfvén wave which then becomes damped by a variety of processes, usually ohmic dissipatation for low $T_{e}$ or Landau damping on the elentrons for higher $T_{e}$, or on the ions via cyclotron damping or Landau damping. It seems quite possible that some electron heating will occur via this process in the planned IDEAL experiment, but there is no hard quantification of this as yet.

For the model problem of cylindrical radial propagation driven by an infinite-in$z$ antenna with specified $k_{z}$, there should be no resistive power loading from the cold 
plasma propagating waves, if they lie outside the Alfvén continuum. If they lie within the Alfvén continuum, resistive power loading for a given model antenna is evidently obtainable even from the cold plasma theory - the numerical models only require a small but finite dissipation to handle the singularity. This does not however tell you where the power dissipated is going - for that you need the kinetic theory.

3.2 $\beta$ limitation for effective interior electron Landau damping. An important limitation of the mode conversion damping shown by Stix [2] is that the mode conversion to a kinetic Alfvén wave that propagates to the interior requires a $\beta$ larger than a minimum:

$$
\beta>m_{e} / m_{i}
$$

If this condition is not met, the mode conversion results in kinetic waves that are reflected and so can be expected to heat only the surface.

The Ross et al $[3$ ]paper gives details on the basic process of mode conversion and resulting Licindau damping. Their calculations are heavily oriented to a specific tokamak model with a given q profile etc, so their specific results are not useable for the Ideal model. But, their numerical evidence for the beta limitation is quite convincing for their specific tokamak model. If the mode conversion layer is at a radius larger than the $\beta=m_{e} / m_{i}$ radius, then only surface electron heating occurs, while if the mode conversion layer is at a radius smaller than the $\beta=m_{e} / m_{i}$ radius, then the mode converts to inward propagating kinetic Alfvén waves that effectively heat the electrons in the interior.

For Ideal parameters at axial position between the antenna and beach, $n=$ $5.10^{13} \mathrm{~cm}^{-3}, B=3 . T, T_{i}=T_{e}=50 \mathrm{ev}$, we appear too low by a factor of 2 or so:

$\beta=n K(T i+T e) 2 \mu_{0} / B^{2}=5.10^{19} \times 1.6 \times 10^{-19} \times 2 . \times 50 . \times 2 . \times 1.3 \times 10^{-6} / 3 .^{2}=.00023$

compared to

$$
m_{e} / m_{i}=1 . / 1836 .=.00054
$$

Thus there is a start up problem for this mode conversion process plus damping to be effective in the plasma interior. Alternatively, the initial electron heating may occur by Landau damping of the slow wave or by electron cycltron resonance heating.

3.3 Calculations of electron absorption in TMX-U and Phaedrus. Porkolab[4], in an 1985 memo re ICRF on Phaedrus and TMX-U, discussed methods whereby electrons would be heated. He mentions the possibility of the absorption in the Alfven resonance. He refers to an even older memo Oct 1983 (which I have not seen) where he calculated the process for TMX-U and concluded therein that the Alfven absorption would effectively "completely absorb" the rf power by electron Landau damping. He does not mention the Stix beta limit. But he remarks that the real problem is a combined axial and radial propagation problem and that this seems not to have been treated in the literature.

He then approximatel: calculates part of this process, which ignores the Alfvén continuum resonance conversion into the KAW, whereby he fixes $k_{\perp}$ and does a WKB type analysis for the axial propagation, using a kinetic model for the waves, and including the 
axial variation of $B_{0}$. This approach might be useful to repeat for the Ideal model. It appears that Porkolab has made some unduly restrictive assumptions about the size of $k_{\perp}$, so his approach may need extending.

3.4 Recent Russian calculations of electron heating for IDEAL. Recent notes from the Russians, Vdovin et al [5], examine the mode conversion and consequent damping on electrons as applied to the IDEAL model, mostly from a purely radial propagation model (infinite-in-z model), and conclude that the electron Landau damping should be able to absorb fully $1 / 2$ of the rf power! This conclusion is similar to what Porkolab claimed for TMX-U. Their analysis is not based on specific numerical integrations of a radially varying model including the kinetic corrections needed for the kinetic Alfvén wave, but rather on examining the propagation and absorption characteristics from infinite medium theory. They also note that the real problem requires the combined radial and axial propagation; they conclude that the electron power will be absorbed before the wave reaches the beach where the ions presumably absorb the rest of the power. They also seem not to be aware of the Stix $\beta$ limit for effective propagation of the KAW into the interior. Their discussion uses a wave notation that requires translation: their fast wave FW is evidently Appert's $S$ wave, possibly also including Appert's global Alfvén mode $A_{1}$; and their slow wave $S W$ is evidently the kinetic Alfvén wave KAW. They describe a variety of scenarios that start with both their FW and SW being excited at the plasma edge by the antenna. (The papers in the literature I have found do not discuss the possibility of their SW (=KAW) being driven by the antenna, only the $\mathrm{FW}=\mathrm{S}$ wave or $F_{1}$ or $A_{1}$ waves). They then describe various conditions whereby no mode conversion takes place, or mode conversion takes place continuously or down to some internal point. It seems that they find conditions for IDEAL where mode conversion is predicted, but it is not clear how they conclude that the electrons will absorb $1 / 2$ of the $\mathrm{rf}$ power.

3.5 Modelling electron absorption via McVey's Antena code. Dimonte et al[6], in a paper about ICRF on TMX-U described modelling the process via the McVey Antena code, which does address the combined radial and axial propagation problem. However, it requires axially uniform $B$ and $n$, so it cannot model the ion heating at the magnetic beach. In this mode the antenna is represented by a finite length structure, so that waves propagating axially away from the antenna represent radiated power, a resistive power drain. The results from TMX-U compare well with predictions from the Antena code for the antennna power loading, so the code seems to accurately handle the axial propagation of the main modes excited in the experiment. The Antena code includes finite $m_{e}$ and some thermal corrections, so it includes Landau damping for example, but it evidently cannot adequately represent the Alfvén continuum mode conversion to the kinetic Alfvén wave. Their calculations indicate that some sizeable fraction of the power coupled would be absorbed by directly heating the electrons, apparently mostly by collisional heating rather than Landau damping.

Despite their calculations that seem to show some direct electron absorption, Dimonte et al make another assessment of the TMX-U experimental results, based on power balance, that seemed favor electron heating from ion drag rather than direct heating by the ICRF.

Motley et al[7] in their IDEAL paper state that in the Antena code, 
"Alfvén resonance effects, important for electron heating, are not, properly treated."

Ferron et al[8] in discussing the Antena code state that

"The code does not account for the Alfvén resonance that could cause wave damping for $\omega$ below $\omega_{c i} . . . "$

Golovato et al[9] in discussing ICRF applied to the TARA tandem mirror central cell show comparisons with Antena for field profiles at $200 \mathrm{~cm}$ away from the antenna. Agreement is quite good for the inner 2/3 of the radial profile. The strong disagreement for the outer $1 / 3$ they associate with "strong edge fields predicted by the code from the $m=1$ fast wave", and they say this mode is apparently not excited in the experiment. It seems to me that a likely candidate for the disagreement would instead be the poor or nonexistent treatment of Alfvén resonance in the Antena code. The body of this paper had no discussion about the effects of Alfvén resonance; they do make a final remark that "mode conversion at Alfvén resonance" is suggested (reference to Myra) to be important.

Tentative conclusions regarding the Antena code:

- It seems that Antena does not properly handle the conversion into the KAW; this requires a kinetic treatment of high $k_{\perp} \rho_{i} \approx 1$.

- Not clear is whether Antena includes some features of the Alfvén continnum absorption or not. Other codes, e.g., Appert's cold plasma models evidently can handle the continuum absorption simply by using a small but finite dissipation. In the cold plasma limit the damping is described by phase mixing with the resulting loading of the antenna insensitive to the value of the dissipation used. The ultimate sink of the absorbed power is not known in this limit, so while power absorption can be predicted, one cannot tell whether it heats ions or electrons or both. In contrast, the 4 papers cited in this section either ignore or dismiss the Alfvén resonance issue entirely in their discussion of Antena.

3.6 Damping from excitation of nearly lossless modes. Karney et al[10], calculate cases where the continuum damping is very weak but nevertheless effective electron Landau damping still results from the kinetic model. Despite the very weak continuum damping, mode conversion is still said to be an essential feature.

Ross et al[3] discuss (but do not give details) of a mechanism whereby waves are excited outside the continuum (presumably the global eigenmode Alfvén waves- GEAW) and thereby have no continuum damping in the cold plasma model, but which evidently do obtain significant damping via electron Landau damping in a kinetic model.

It would seem that in these latter cases, the power loading on an antenna would not be obtained in a cold plasma model- the models are both infinite-in-z radial propagation models so no power loading should result from waves propagating axially away from the antenna, and the damping from the continuum is either very weak (Karney) or nonexistent (Ross). This process needs elucidation. Karney et al seem to be using both the very weak continuum damping and mode conversion in the continuum. Ross et al possibly are claiming that a wave just outside the continuum can still somehow be mode converted and thus get effective Landau damping. Or, it may be that both of these cases are relying on very 
weak dissipation in the cold limit, so obtair ing large standing waves, nearly eigenmodes, set up with a small investment in external power circulation. The Landau damping on the electrons possibly then would be calculable via a perturbative treatment (Nevins proposal) without considering any mode conversion process - the relative weakness of the Landau damping is offset by the small price for external power circulation so that effective electron heating might still occur.

The modes used here, either within the Alfvén continuum but very weakly damped in the cold plasma model, or just outside the Alfvén continuum and thus not damped at all in the cold plasma model, might be able to be diriven, even may be unavoidably excited, by the Ideal antenna, so this concept might be worth further elucidation.

3.7 Mode description, taken mostly from Appert et al Phys Fluids 1984 [11]. Appert et al use cold plasma theory and $m_{e}=0$ to give a thorough description of the waves present in a cylindrical plasma immersed in a uniform $B_{z}$. They give detailed examples of both the sharp boundary density model and a parabolic density model. The latter has the Alfvén continuum present. They discuss:

1) higher radial eigenmodes of the fast magnetic waves. These modes have a finite cutoff, where $\omega / k_{z} \rightarrow \infty$ and do not propagate for frequencies below this cutoff. Both $m= \pm 1$ occur and act very similarly for small $k_{z}$.

2) lowest radial eigenmodes of the fast magnetic waves. Both $m= \pm 1$ versions of these modes persist for $\omega<\omega_{c i}$ down to zero frequency (no cutoff). Here only as $\omega / \omega_{c i} \rightarrow 0$ are the $m= \pm 1$ branches similar.

- The $m=+1$ mode, labeleld $F_{1}$ in Appert et al, passes right through $\omega=\omega_{c i}$ without strong change.

- The $m=-1$ mode asymptotically approaches $\omega_{c i}$ as $k_{z} \rightarrow \infty$. This mode Appert labels as S for "Surface" mode, although he notes that for low $k_{z} a$ its field structure is global, only changing to a "surface" character of evanescent decay away from the plasma boundary for sufficiently high $k_{z}$. This mode start out at low frequency as a fast mode, $\omega / k_{z}>v_{A}$, just like the $m=+1$, but at larger $k_{z}$ changes to a slow mode $\omega / k_{z}<v_{A}$; the transition from fast to slow is smooth. This mode appears to exist entirely within the Alfvén continuum.

3) A spectrum of modes labeled as Global Eigenmode Alfvén Waves (GEAW). These modes lie just below the Alfvén continuum. Both $m= \pm 1$ exist at nearly the same location. Also higher radial mode versions of these GEAW also appear in the same vincinity, very tightly packed. These GEAW modes exist only for $\omega<\omega_{c i}$ and have $\omega / k_{z}<v_{A}$, i.e., they are slow modes. These may be the same as the slow modes discussed by Paoloni, Phys. Fluids 1975, although the latter uses finite $m_{e}$.

The $m=-1$ lowest radial "fast" eigenmode S discussed above merges with or is only minimally separated from the lowest GEAW, labeled as $A_{1}$, at some finite $k_{z}$. It appears that the Ideal antenna chosen parameters will come close to driving a wave close to the merging point:

$$
\omega / \omega_{c i} \approx 0.7
$$




$$
k_{z} a=0.4 \times 4 .=1.6
$$

where $\mathrm{a}$ is the plasma radius. The precise location in $\omega-k_{z}$ space of this merging point is of course dependent on the plasma and antenna parameters, $n, B, a, k_{z}$, but it appears that Appert's plots are for the conditions

$$
\frac{\omega_{c i} a}{V_{A}}=2.0
$$

not too different than those planned for Ideal. It may be worth attempting to match this point:

- the merging point seem less sensitive than other parts of the dispersion curve for $S$ to the details of the density profile: compare Appert's plot for the sharp boundary to his plot for the parabolic profile. So matching this point may give the best excitation of the $\mathrm{S}$ mode.

- matching this point may best match conditions for absorption in the interior of the plasma- it is very close to the $r=0$ Alfvén continuum curve.

- alternatively, driving the antenna close to this point may also or instead succeed in exciting the $A_{1}$ GEAW which actually lies below the continuum. The paper of Ross et al indicate that this may be favorable for electron Landau damping, even though these modes do not experience mode conversion in the continuum and thus see no damping in the cold plasma model.

3.8 What tools do we need for calculation of electron heating? The optimum code would have the following:

1) Both radial and axial propagation of the waves; this should include waves propagating outward from the axial antenna region.

2) allow for axial variation, $B(z)$. For electron heating we may not need to include the ion $\omega=\omega_{c i(z)}$ resonance layer. We do need some appreciable region of axial variation in $\mathrm{B}(z)$ and hence in $k_{\|}(z)$ to observe the region of optimum electron Landau damping.

3) a sufficiently robust kinetic treatment of the waves so as to be able to treat $k_{\perp} \rho_{i} \approx 1$ which is necessary for modelling the KAW. The models used here presumably could be similar to those used in the paper by Ross et al[3], simplified to remove toroidal terms.

All of the above does not exist in one package.

Antena has 1) but not 2) or 3). It evidently does include electron Landau damping on the waves that it includes.

Garfield[12] evidently has 1) and 2). It is evidently based on the cold plasma equations, with small dissipation added ala Appert. This code could tell us something about the combined axial and radial propagation including $B(z)$, and would say something about the Alfvén continuum resonance absorption (in the cold plasma limit). It would tell us nothing about the ultimate sink of the power absorbed at the Alfven resonance. Since it uses the cold plasma equations it would not give the electron Landau damping on any waves included. 
A possible scenario would use a combination of codes:

- first use Antena and Garfield(if available) to scope out some gross features of the r-z propagation, including locations of Alfvén resonance absorption (in Garfield, questionable with Antena). Electron Landau damping on the popagating cold plasma modes would be obtained from Antena. The changes in $k_{\|}(z)$ would appear in Garfield.

- second, develop a kinetic code that included the KAW conversion but limited to radial propagation (fixed set of $k_{\|}$from an infinite-in-z antenna), uniform B. This kinetic model would be used to model the mode conversion to KAW and subsequent Landau damping process at several axial locations as determined by the scans with Antena and/or Garfield.

The attached pages include:

- 2 plots showing the dispersion characteristics of the modes in cold plasma limit, from Appert et al Phys Fluids 27 (1984)

\section{References}

[1] A. Hasegawa, L. Chen, Phys. Fluids 191924 (1976)

[2] T. H. Stix, in Heating in Toroidal Plasmas, Vol. 2, 631, (1980)

[3] D.W. Ross, G. L. Chen, and S. M. Mahajan, Phys. Fluids 25652 (1982)

[4] M. Porkolab, "Electron Heating via ICRF Waves to Enhance the Plug Potential" unpublished memo, Aug 9, 1985

[5] V,L. Vdovin, Yu. Sokolov, "Analysis of ICRF problems in IDEAL Device, I" 18 May 1993, Notes obtained from S. Cohen

[6] G. Dimonte, A.W. Molvik, J. Barter, W.F. Cummins, S. Falabella, P. Poulsen, T. Romesser, Nuclear Fusion 271959 (1987)

[7] R.W. Motley, R. Majeski, S.A. Cohen, M. Diesso, and J.R. Wilson, "ICRF Coil for the IDEAL Plasma", Draft copy from recent rf meeting in Boston, March 1993

[8] J.R. Ferron, S. N. Golovato, N. Hershkowitz, and R. Goulding, Phys. Fluids, 301869 (1987)

[9] S. N. Golovato, K. Brau, J. Casey, M.J. Gerver, S. Horne,J. Irby, J. Kesner, B. Lane, J. Machuzak, R. Myer, R.S. Post, E. Sevillano, and L. Wang, Phys. Fluids B, 1851 (1989)

[10] C.F.F Karney, F.W. Perkins, and Y.-C. Sun, Phys. Rev. Let. 421621 (1979)

[11]K. Appert, J. Vaclavik, and L. Villard, Phys. Fluids 27432 (1984)

[12] M. W. Phillips "Numerical Solutions of ICRF Fields in Axisymmetric Mirrors", in Radio Frequency Plasma Heating, Sixth Topical Conference, Callaway Gardens, GA, AIP Conference Proceedings, 1985 

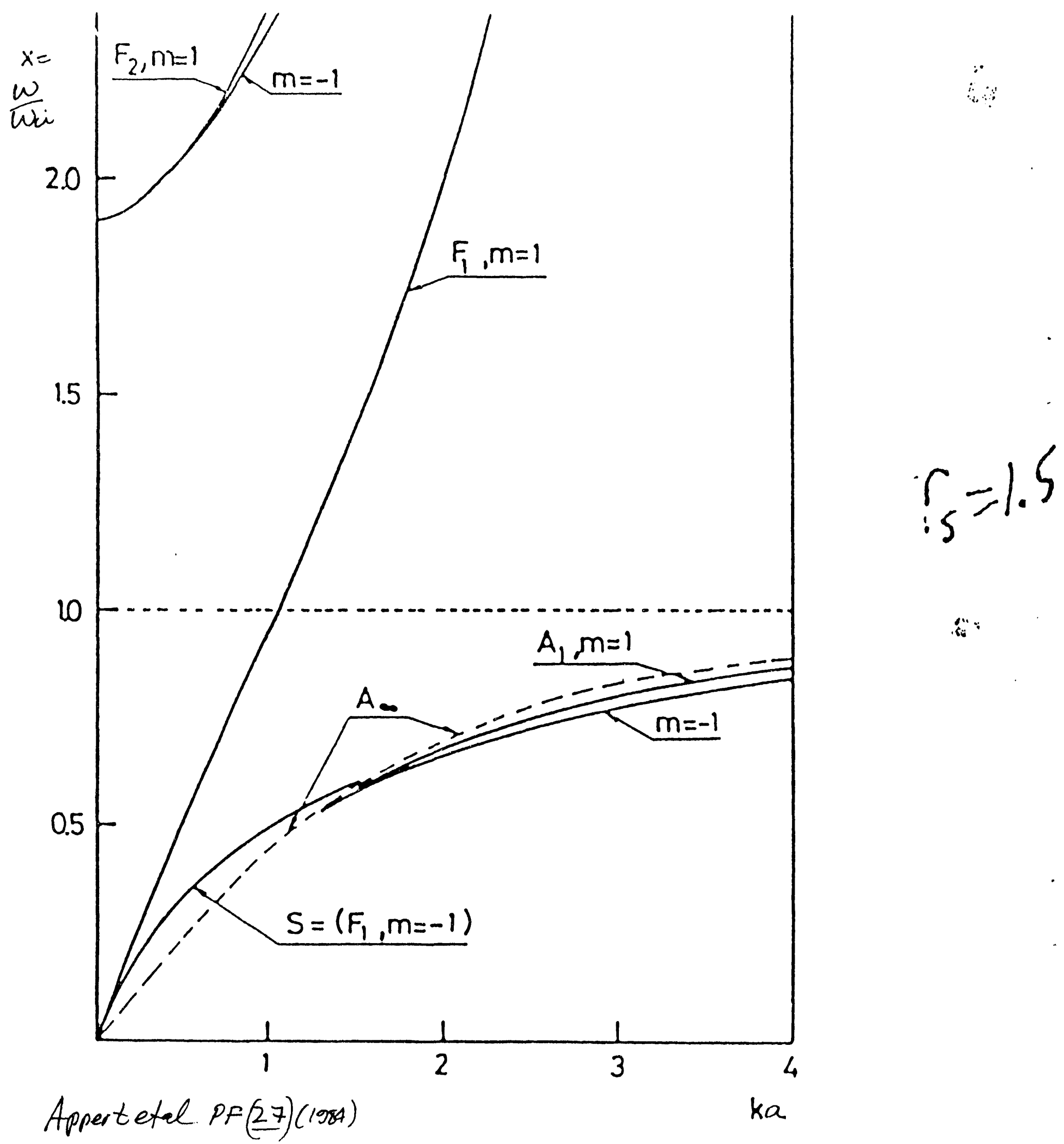

:

FIG. 1. The frequency spectrum $x=\omega / \omega_{c i}$ of a currentless plasma cylind with uniform density as a function of the axial wavenumber $k$. For the $t x$ azimuthal wavenumbers $m= \pm 1$, the lowest radial modes of the fast ma netosonic wave are designated as $F_{1}$ and $F_{2}$, those of the Alfvén wave as $A$ The broken line $A_{\infty}$ represents the accumulation point of the eigenfreque: cies of the Alfvén wave. The symbol ${ }_{3-8} S$ stands for "surface eigenmode." 


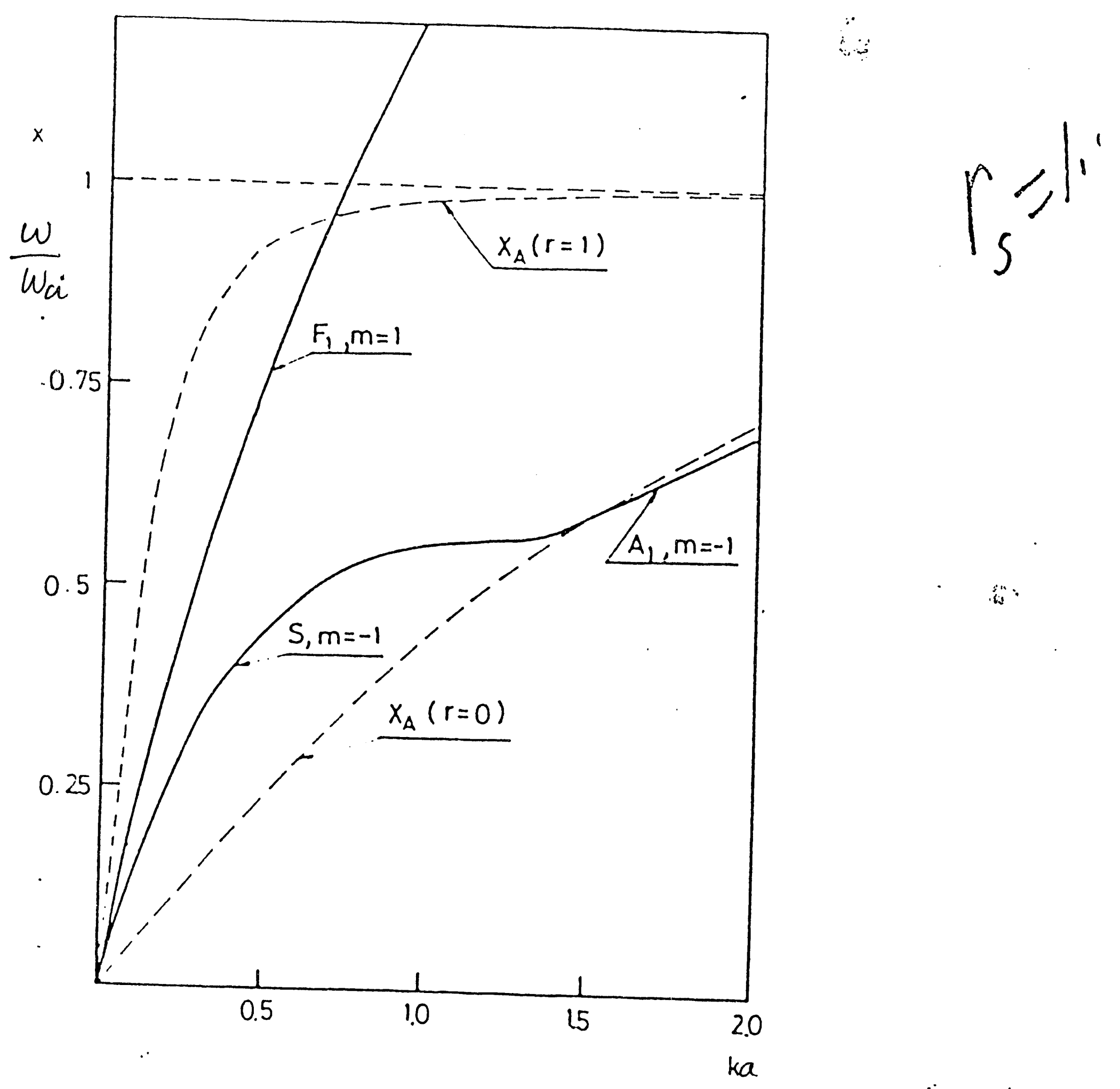

FIG. 4. The frequency spectrum $x=\omega / \omega_{c i}$ of a currentless plasma cylind with parabolic density pirofile as a function of the axial wavenumber $k$. Tr upper and lower bounds of the Alfién continuum are shown with broke lines, $x_{\mathrm{A}}(r=1)$ and $x_{\mathrm{A}}(r=0)$, respectively. Only the most importar modes, $F_{1}, S$, and $A_{1}$ are shown.

436

\section{Appert, etal}

Phys. Fluids, Vol. 27, No. 2, February 1984 


\section{Electron Cyclotron Resonance Heating: Proposed Geometry of Injection}

G. R. Smith

\subsection{Summary}

Propagation and absorption of electron-cyclotron power in the IDEAL plasma can be predicted with considerable confidence by standard geometrical-optics techniques. Modelling work performed for the TMX-Upgrade Experiment suggests that high absorption efficiency could be achieved by a simple scenario for launching power towards the IDEAL plasma.

The electron-heating scenario studied for TMX-U (but never tested experimentally) has the following characteristics. Power generated by $18 \mathrm{GHz}$ Klystrons is launched through microwave horns in the extraordinary mode from the high-magnetic-field side of the electron-cyclotron resonance. A report of the study is shown as Appendix 4A. Results of ray-tracing calculations indicate that power is guided down the magnetic field to the cyclotron resonance (at $B=0.64 \mathrm{~T}$ ), where complete absorption occurs. Guiding is effective at trapping power within the plasma over a broad range of plasma densities, characterized by on-axis values of $\left(\omega_{\mathrm{pe}} / \omega_{\mathrm{ce}}\right)^{2}$ in the range 0.05 to 1 .

In IDEAL, a higher magnetic field of approximately $B=2.5 \mathrm{~T}$ requires $70 \mathrm{GHz}$ gyrotron power. Efficient absorption is predicted due to very good trapping of power launched towards the plasma from the high-field side (i.e., smaller $\mathrm{z}$ than the cyclotron-resonance location).

Although ray-tracing calculations for IDEAL have not been performed, guiding similar to that calculated for TMX-U is expected because IDEAL has a value of the relevant dimensionless parameter $\left(\omega_{\mathrm{pe}} / \omega_{\mathrm{ce}}\right)^{2}$ of approximately unity, which was in the range included in the TMX-U study. 


\section{A Power Deposition by $18 \mathrm{GHz}$ ECH in the TMX-U Central Cell}

MEMORANDUM

June 10, 1986

PT606075

To: M-Division Physicists

From: Gary R. Smith and Reed A. James

Subject: Power Deposition by $18 \mathrm{GHz}$ ECH in the TMX-U Central Cell

\section{Summary}

We have used the ECH package in the MERTH tandem-mirror modeling code to calculate ray trajectories and power-deposition profiles for the $18 \mathrm{GHz}$ launching system in the west end of the TMX-U central cell. We find that

1. Of the power that enters the plasma polarized in the extraordinary mode, more than $80 \%$ is absorbed, independent of on-axis density in the range $0.2 \times 10^{12} \leq n_{0} \leq$ $4.0 \times 10^{12}$.

2. The power is deposited in a narrow swath across the center of the plasma; the power deposition therefore has strong azimuthal asymmetry.

3. Power deposition is highest near the edge of the plasma that is closest to the launching horn but is also high near the opposite edge of the plasma.

4. The on-axis power deposition is enhanced 4 to 5 times over a (hypothetical) case of uniform deposition. The enhancement extends about $4 \mathrm{~cm}$ from the axis.

\section{Models}

The MERTH code provides a very good approximation of the TMX-U magnetic field to the ECH package. The field is based on the long, thin approximation and on EFFI calculations of a near-axis field line. [The EFFI calculation is identified as "upic12(upic08)" and had the goal of making the field at $z= \pm 602 \mathrm{~cm}$ equal to $5 \mathrm{kG}$.] The ECH package thus has an accurate description of the fanning of the field lines near the end of the central cell.

The density and temperature of the central-cell electrons are assumed to depend only on the "radial" flux $\psi$ and are therefore uniform along field lines and around the azimuth. The density is zero outside the flux tube that has $r=25 \mathrm{~cm}$ at $z=0$. Inside that flux iube, the chosen $\psi$-dependence leads to the density and temperature profiles shown as a function of $x$ (north-to-south) and $y$ (bottom-to-top) in Fig. 1. 
The $18 \mathrm{GHz}$ central-cell heating system includes 4 horns, two in the west and two in the east. In this memo, we model one of the horns in the west. The other west horn illuminates the opposite (north) side of the plasma and produces power-deposition profiles like those shown here but with $x \rightarrow-x$. The two east horns have a slightly different location relative to the plasma and a different half-power angle than the west horns. Power-deposition profiles for the east horns should be qualitatively similar to the profiles for the west horns, to which we restrict ourselves here.

The microwave beam emanating from one of the west horns is modeled by a bundle of rays launched from the location $(x, y, z)=(17,0,325) \mathrm{cm}$. The central ray of the bundle is aimed toward $(0,0,296) \mathrm{cm}$. The rays are distributed within a circular cone of divergence angle $=20^{\circ}$. The total number of rays is 121 . The power profile of the beam has a Gaussian shape with a half-power angle of $10^{\circ}$.

All of the calculated values of power deposition are linearly proportional to the incident power that enters the plasma in the extraordinary mode from the single horn modeled here. We have taken that power to be $10 \mathrm{~kW}$ in the present calculations, and our results should be scaled down by a factor 0.35 , because, of the $10 \mathrm{~kW}$ that can be delivered to each horn by the Klystron, $7 \mathrm{~kW}$ will leave the horn, and only about $3.5 \mathrm{~kW}$ will enter the plasma in the extraordinary mode.

The ray-tracing equations use the cold-plasma approximation. The absorption calculation assumes a Maxwellian plasma.

\section{Results}

In Figs. 2 and 3 we show two views of the ray bundle impinging on the plasma and getting absorbed. Only 13 of the 121 rays are shown, the central ray and 12 rays with the maximum divergence angle of $20^{\circ}$. The numbers $1,2,3,4$, and 5 along the rays indicate, respectively, where $20,40,60,80$, and $99 \%$ of the power has been absorbed. Note the strong refraction that tends to trap the power and guide it down the field to the cyclotron resonance, where the power is totally absorbed. Also note that a few rays at the outer edge of the bundle are not absorbed.

We have kept the on-axis electron temperature $=0.1 \mathrm{keV}$ in all of our runs but have used on-axis densities of $0.2,0.5,1.0,2.0$, and $4.0 \times 10^{12} \mathrm{~cm}^{-3}$. All of these densities lead to qualitatively similar ray trajectories and power-deposition profiles.

The azimuthal and radial distribution of the deposited power is shown in the next few figures. This two-dimensional representation of the power is derived by integrating the power per unit volume along field lines. We then report $P(r, \theta)$, the power per unit area deposited on the field line with polar coordinates $r$ and $\theta$ at the central-cell midplane $(z=0)$.

As one can see from Figs. 4, 5, and 6, the deposited power is restricted to a narrow swath across the center of the plasma (near $y=0$ ), because the microwaves reach only 
that part of the plasma. The east horns, not treated here, would deposit power in a narrow swath near $x=0$, because the fans at the two ends of the central cell are rotated by $90^{\circ}$ with respect to each other.

The highest power deposition occurs on the side of the plasma closest to the horn. There is also a peak in the deposition profile near the opposite edge of the plasma, where the refraction of the rays leads to long path lengths in the edge region (see Fig. 2). The contour levels used in Figs. 4, 5, and 6 are given in the upper right corner of each figure and indicate power per unit area in $\mathrm{kW} / \mathrm{cm}^{2}$.

Figure $7(a)$ shows the azimuthal integral of the deposited power, i.e.,

$$
\int_{0}^{2 \pi} d \theta P(r, \theta) .
$$

This quantity may be the appropriate indicator of the effect of the $\mathrm{ECH}$ power on electron temperature, since the azimuthal variations in $P(r, \theta)$ are likely averaged away by plasma rotation and/or by azimuthal particle drifts. Note that near $r=0$, the azimuthal integral of the power is about 4 times larger than for $r>10 \mathrm{~cm}$. The $18 \mathrm{GHz}$ power is therefore expected to heat the near-axis plasma much better than a uniform-deposition estimate would indicate.

In Fig. $7(\mathrm{~b})$, we show the cumulative power deposition as a function of $r$, namely,

$$
\int_{0}^{r} r^{\prime} d r^{\prime} \int_{0}^{2 \pi} d \theta P\left(r^{\prime}, \theta\right) .
$$

At $r=25 \mathrm{~cm}$, this quantity gives the total power deposited in the plasma, which is close to the $8 \mathrm{~kW}$ ( $80 \%$ of incident power) shown at $r=20 \mathrm{~cm}$ at the right edge of Fig. 7 (b). Note that $\approx 30 \%$ of the incident power is absorbed in the core $(r<10 \mathrm{~cm})$.

Figure 7 was calculated for an on-axis density of $1 \times 10^{12}$, but at other densities, the integrals of the power deposition are nearly the same. The only noteworthy differences are that at higher densities the on-axis deposition rises to 5 times the uniform-deposition value, and that $40 \%$ absorption occurs in the core. 


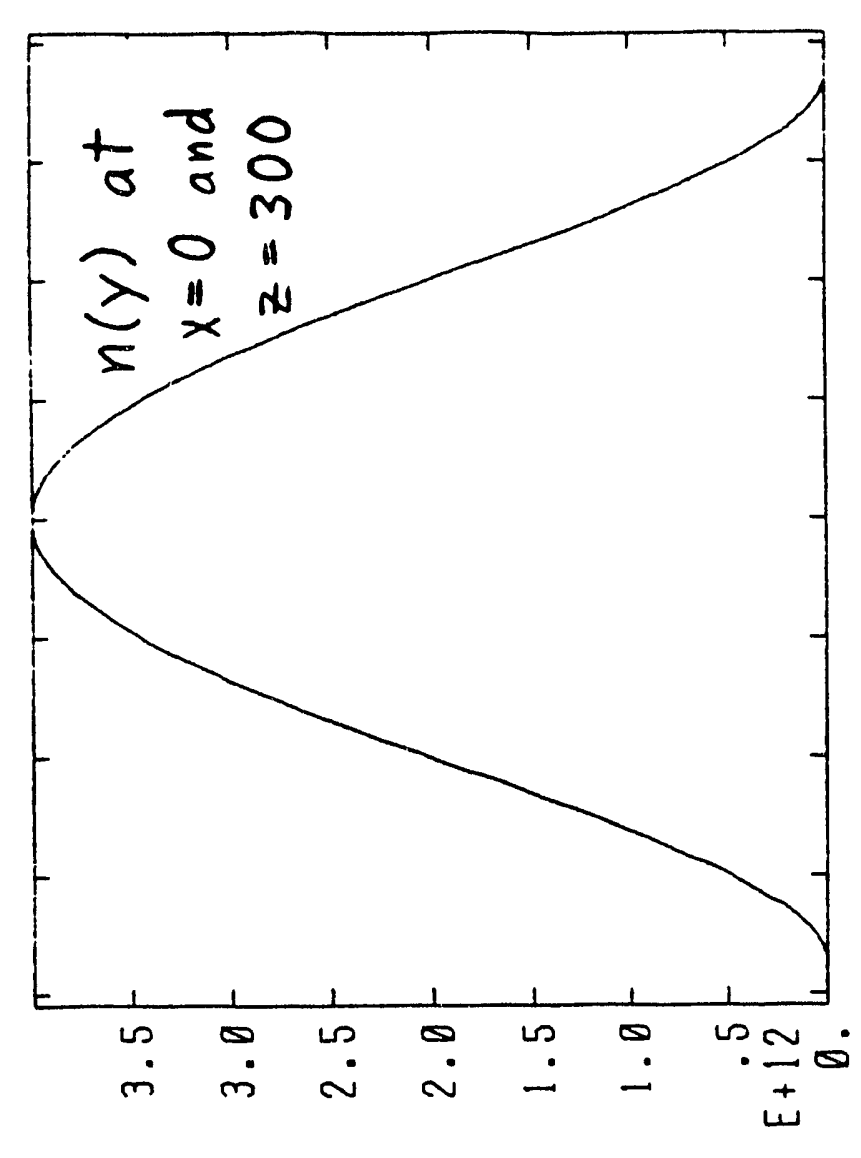

(E・ลพว/I) U

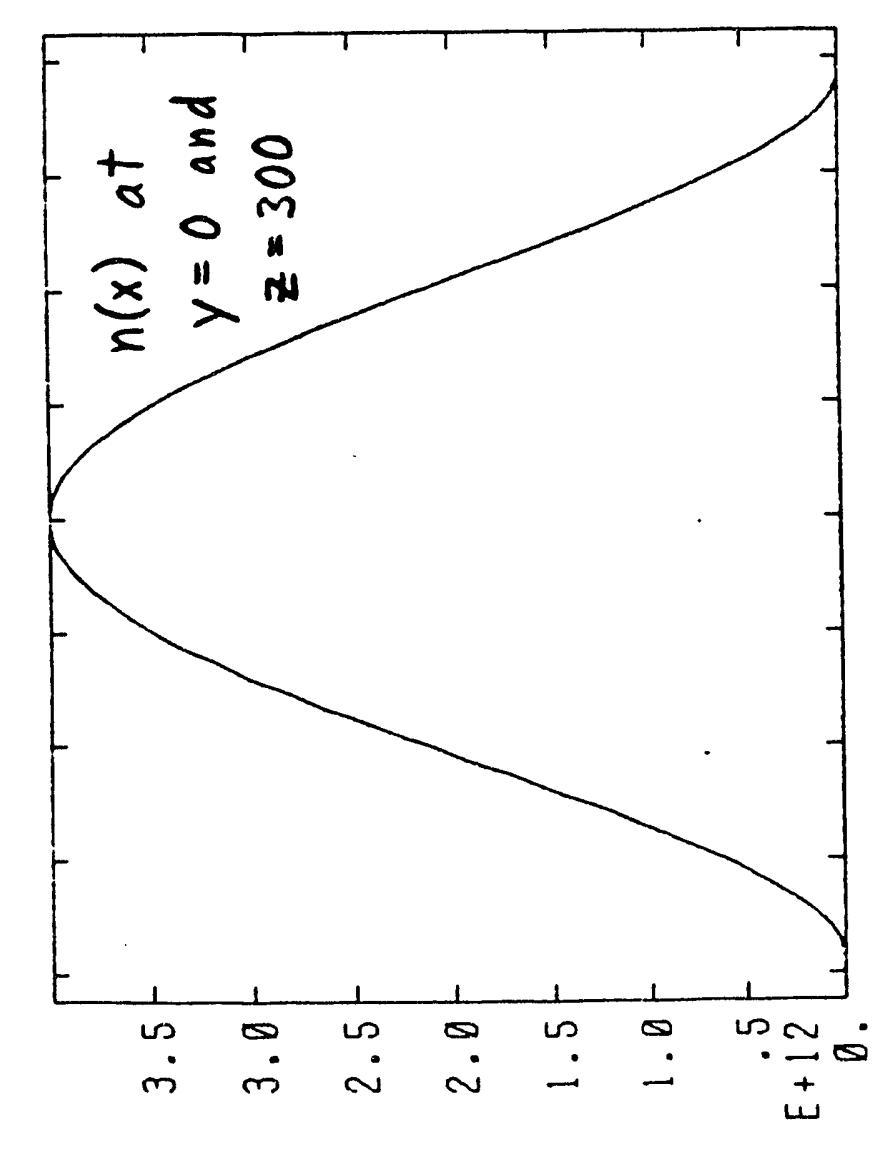

$(\varepsilon \cdots$ แ

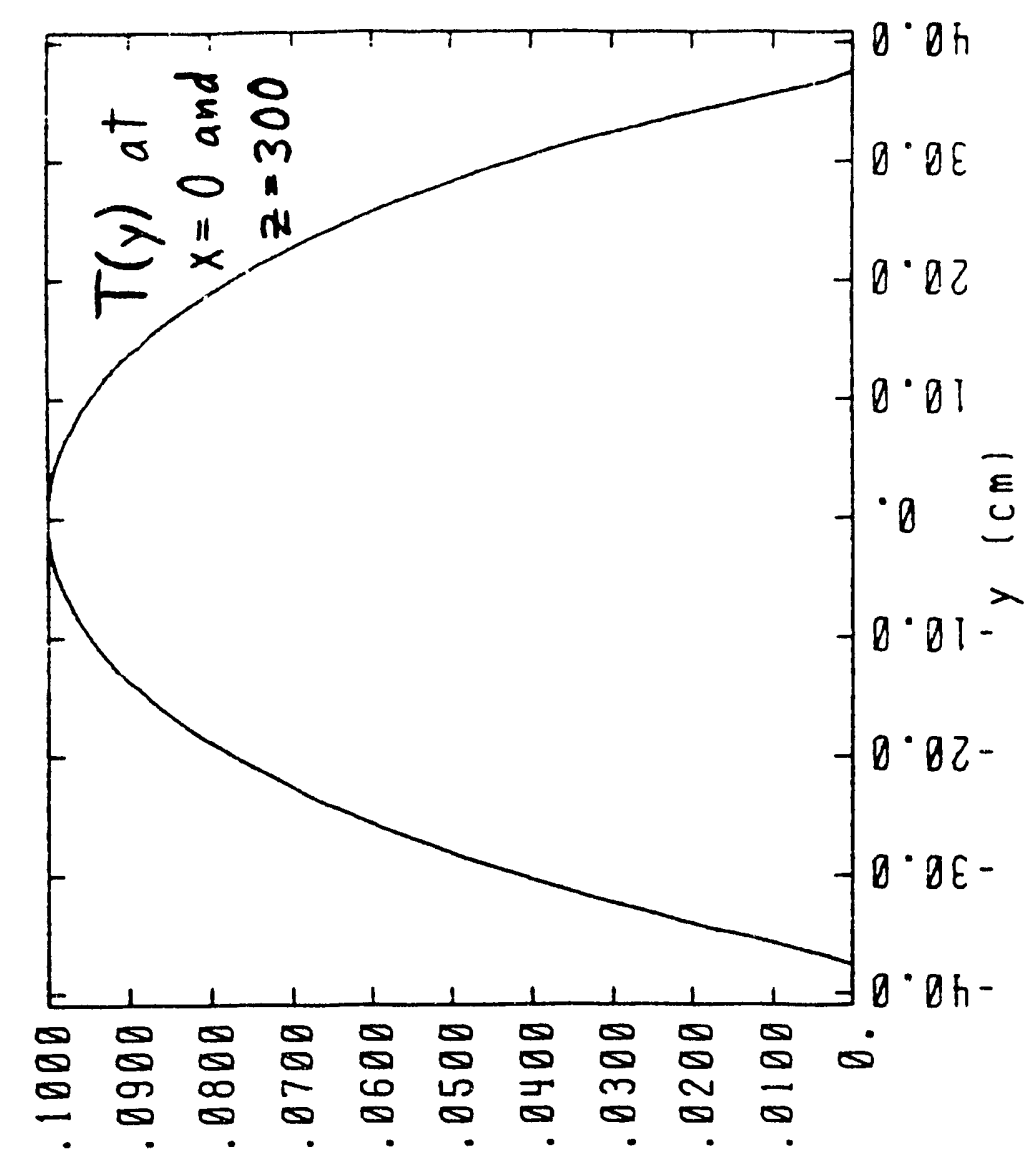

$(\Lambda \partial y) \perp$

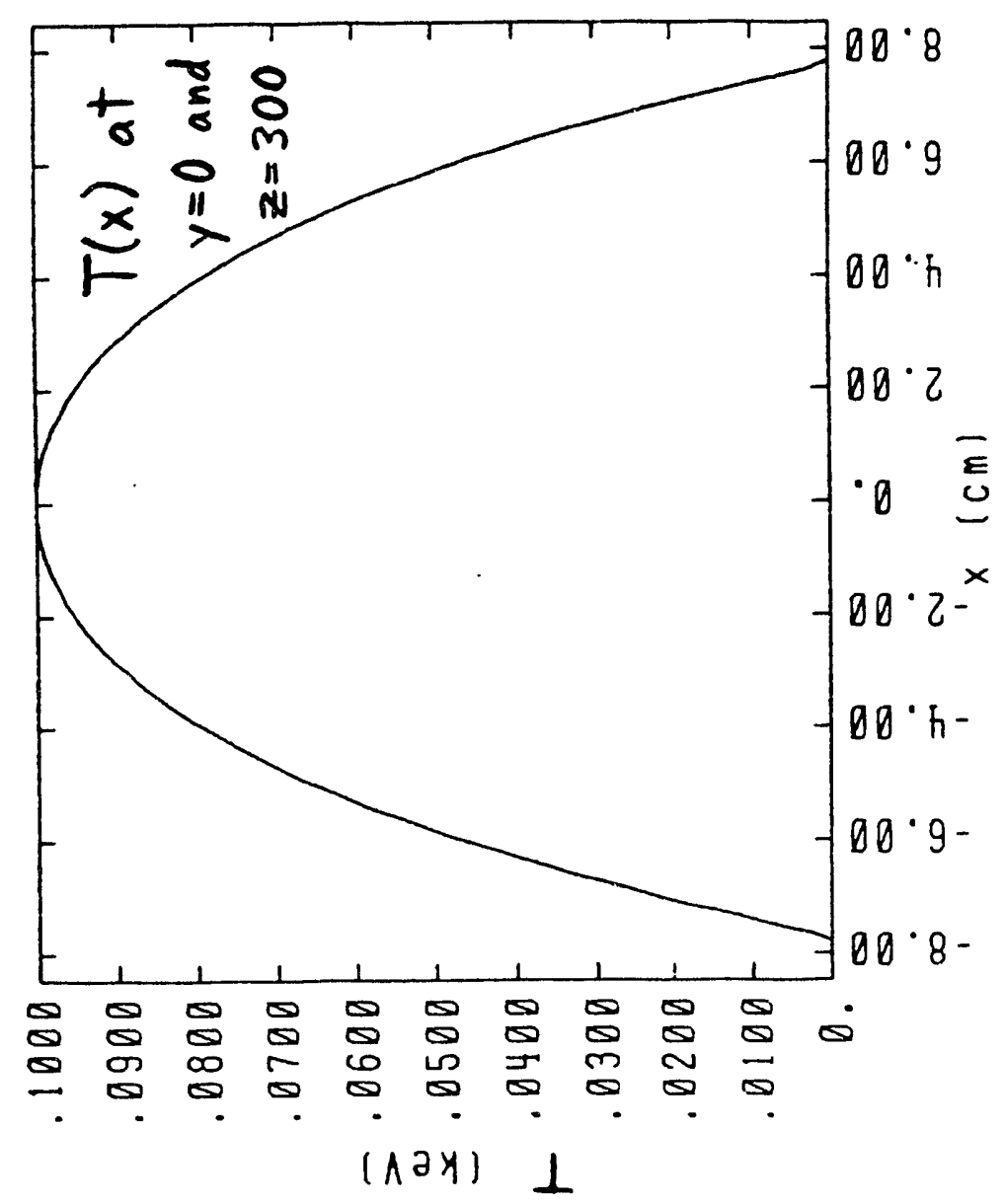

Fiq. I 


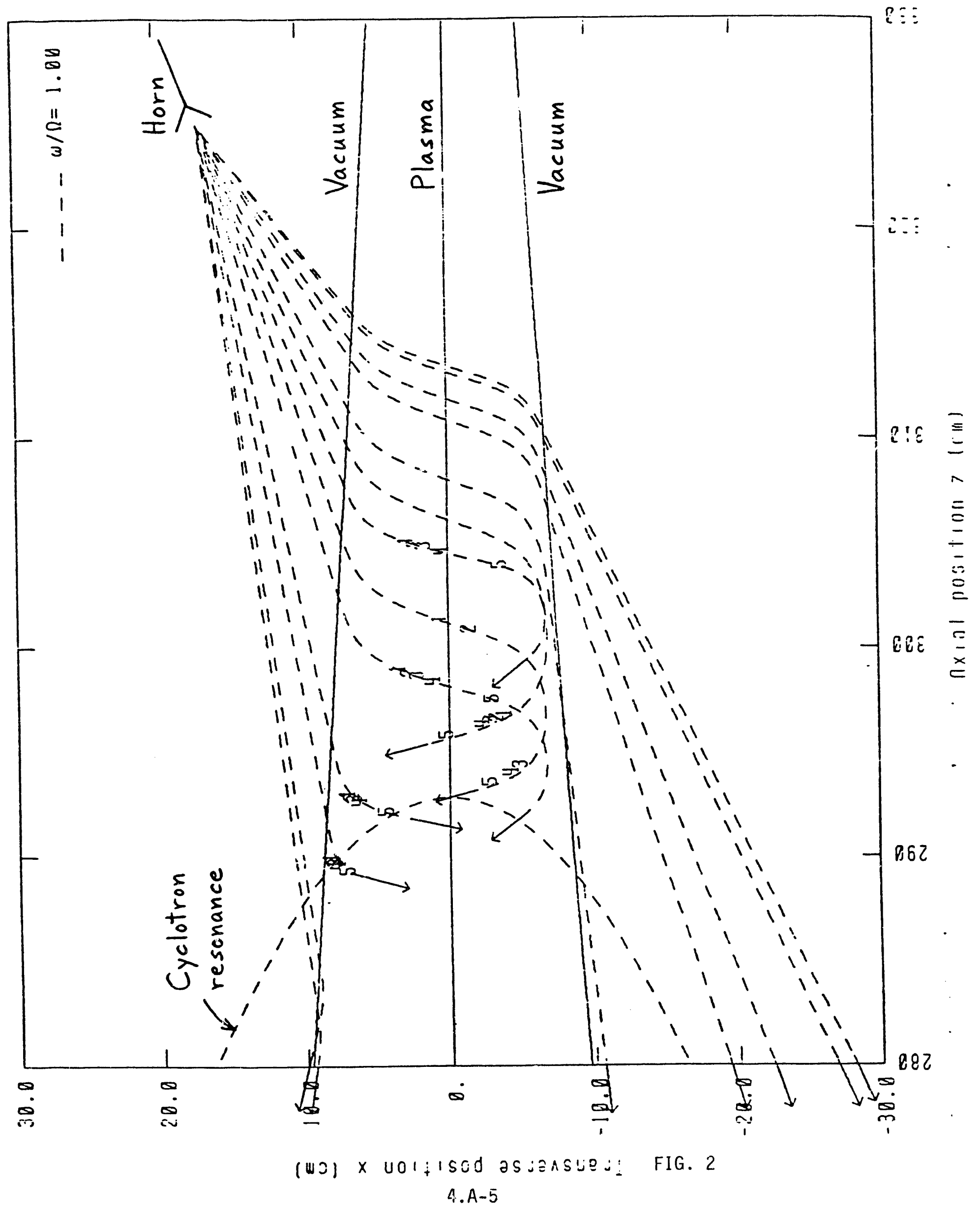




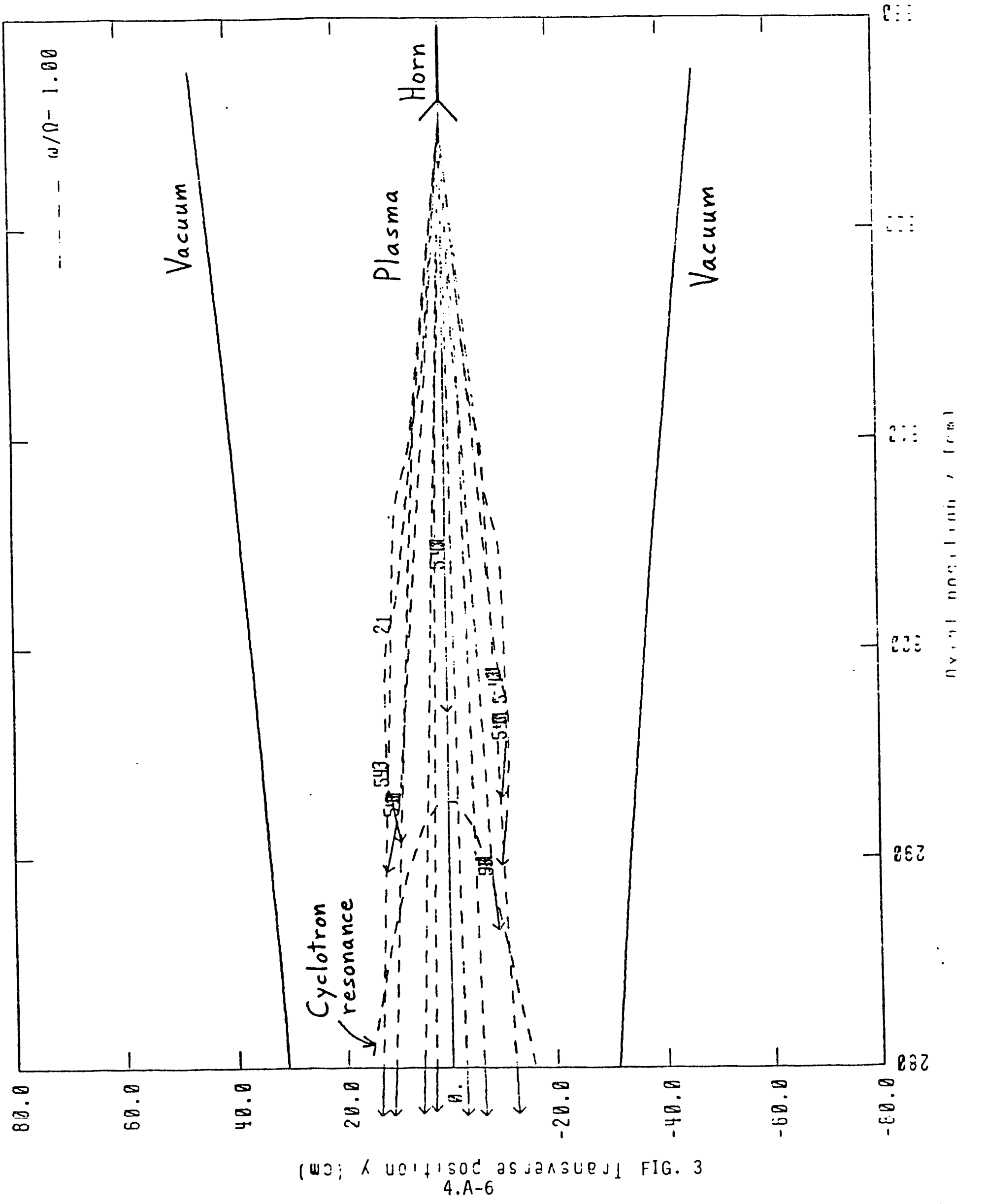




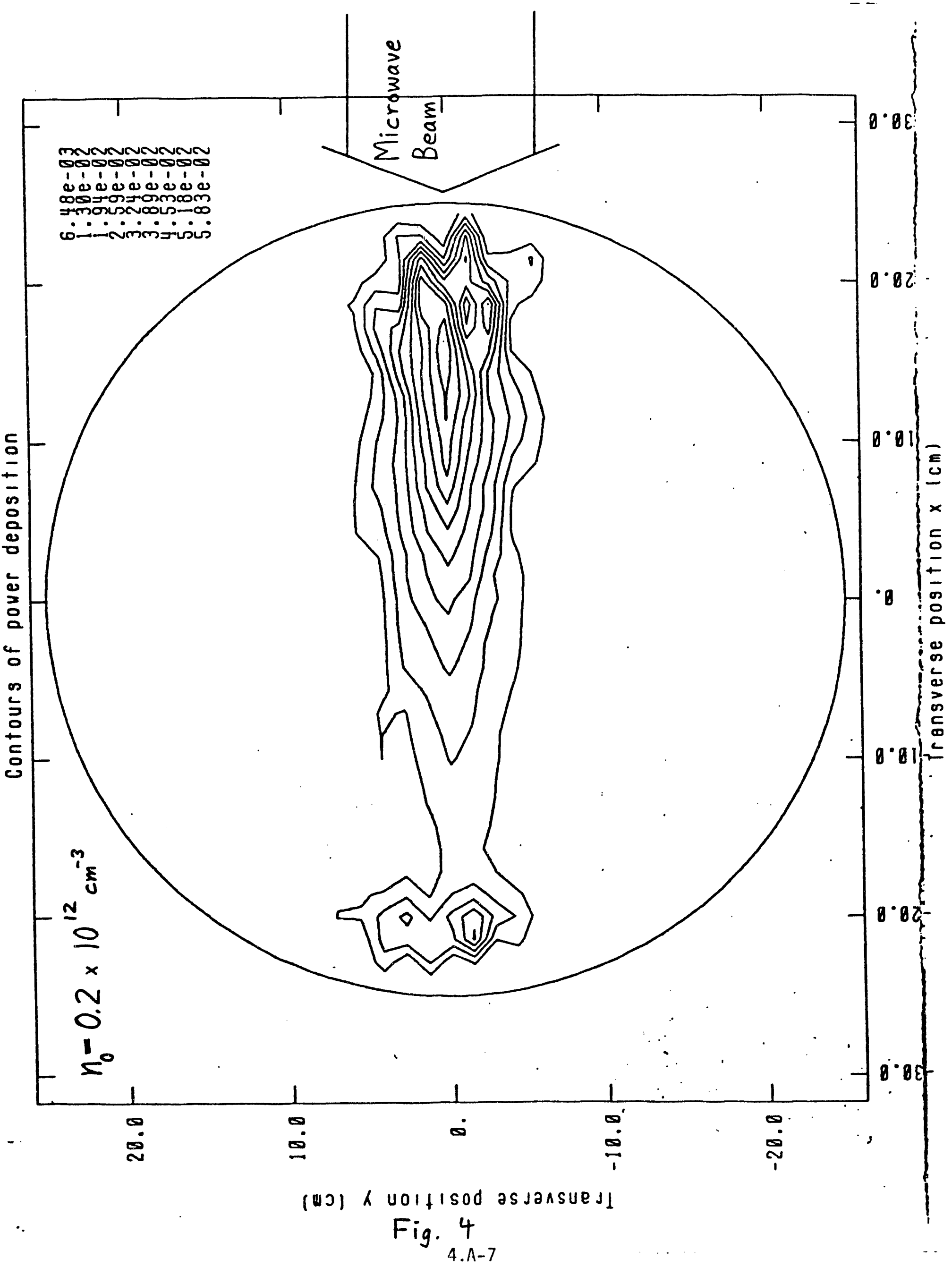




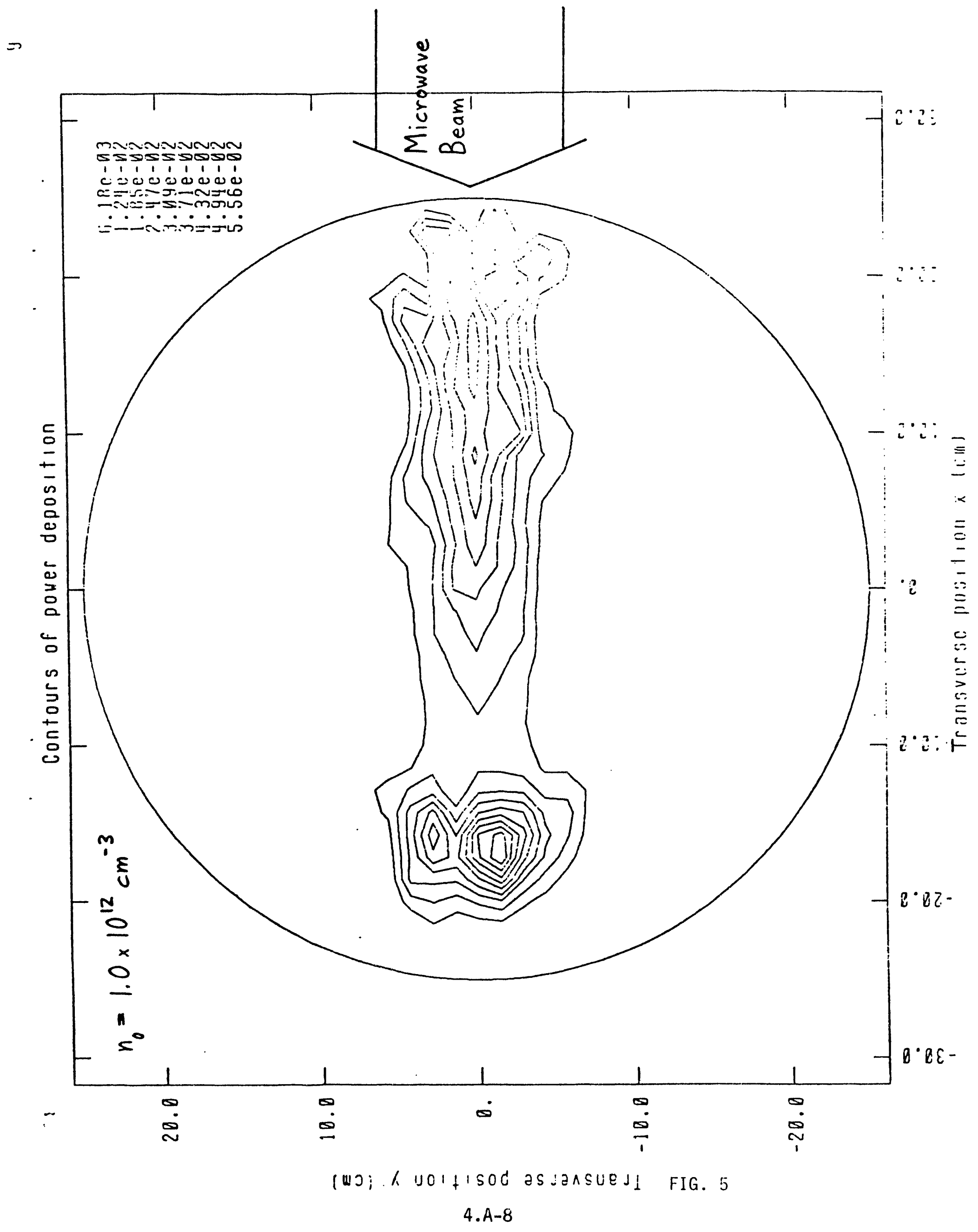




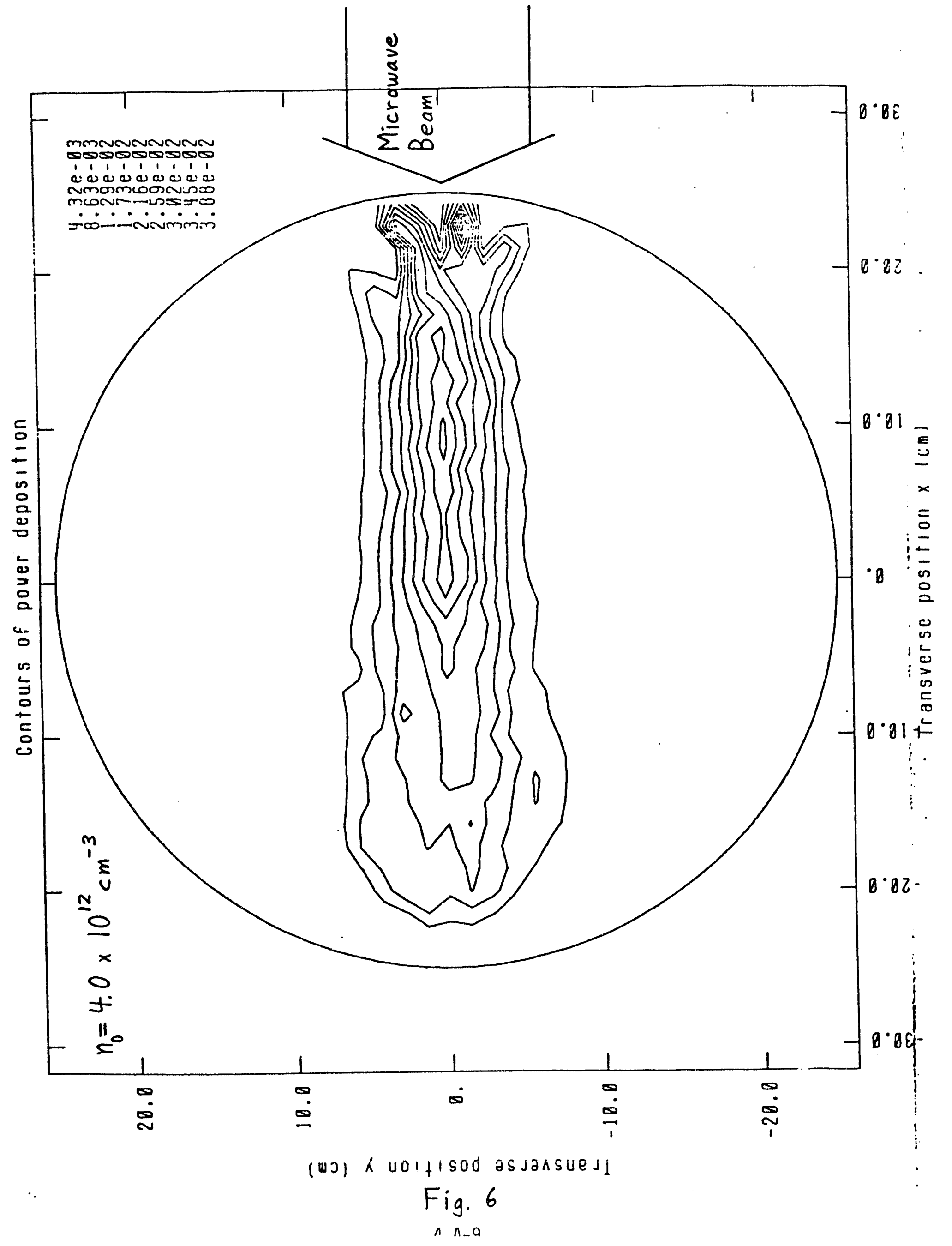




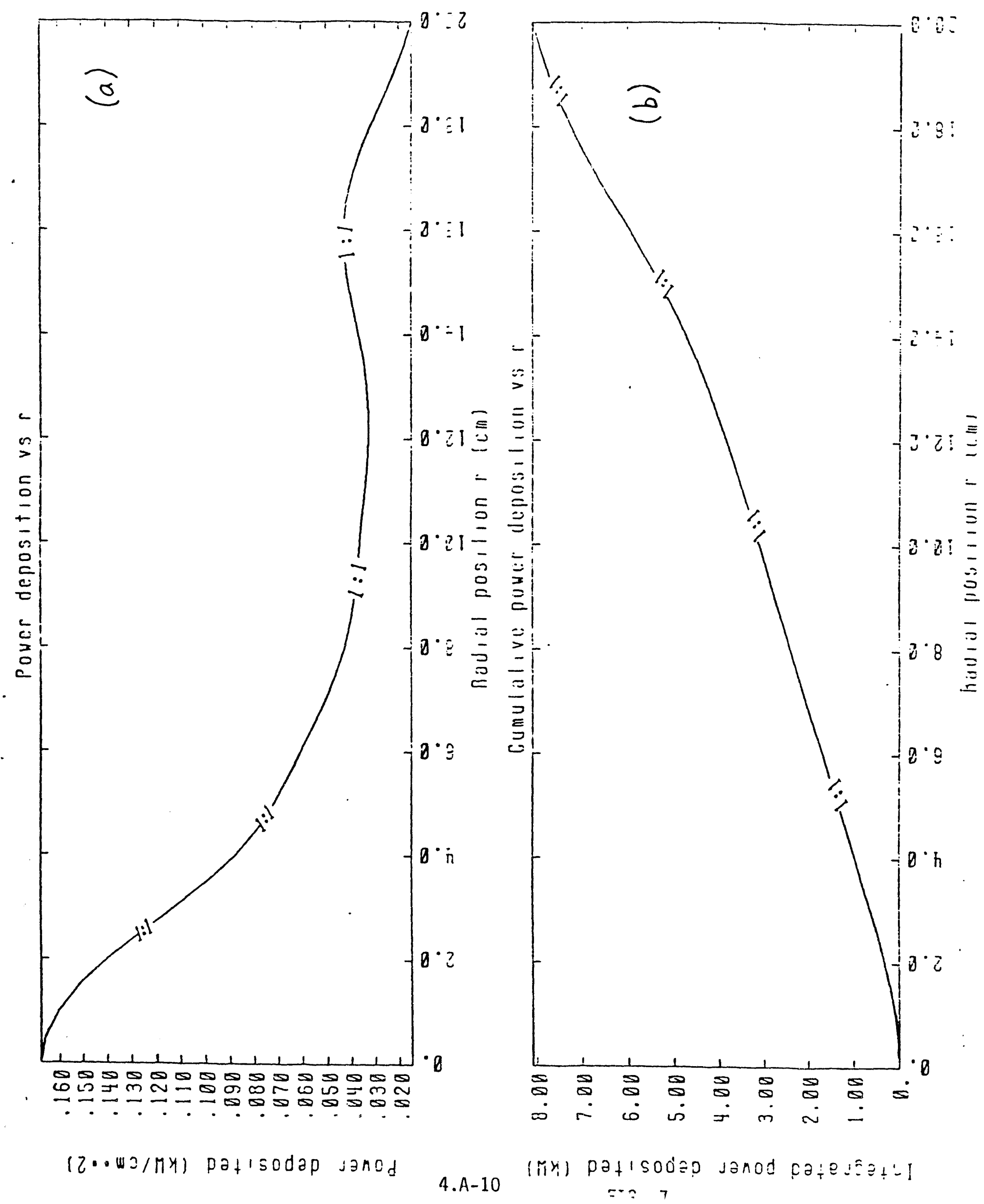




\section{2-D Fluid Transport Calculations with UEDGE}

T.B. Kaiser and T.D. Rognlien

\subsection{Introduction}

In this section we report on simulation results from the 2-D fluid transport code UEDGE that will provide information on the radial as well as axial structure of the plasma column. We will consider the effects of recycling at the end plates edge, fueling at the midplane from a gas-box-like edge source, and the power to a radial limiter located near the midplane.

\subsection{The UEDGE Code}

The UEDGE code is a composite of algorithms developed in two earlier fully implicit codes, NEWEDGE ${ }^{1}$ and LEDGE, ${ }^{2}$ and is available as a public file on the NERSC C-90 computer. The physics description in UEDGE is similar to that in the partially implicit code $\mathrm{B}^{3}$ with some exceptions noted later. The equations solved are for ion continuity, parallel momentum, electron energy, ion energy, and neutral gas continuity for one or two species. The plasma equations assume classical transport parallel to the magnetic field lines with flux-limited diffusion for the long mean free path regime. The electron and ion thermal fluxes are limited to $0.15 n \mathrm{nT}_{t}$ where $v_{t}$ is the thermal velocity; the ion viscosity is limited to $n T_{i}$. The plasma transport across $B$ is described by anomalous diffusion coefficients for particles (coefficient $D$ ), electron energy (coefficient $\chi_{\mathrm{e}}$ ), and ion energy (coefficient $\chi_{\mathrm{i}}$ ).

For the neutral gas, we can combine the Franck-Condon and chargeexchange neutrals into a single species that diffuses axially and radially with a mean free path defined by the net charge-exchange and ionization frequencies. The gas diffusion is proportional to the gas pressure, with the gas temperature assumed to be the same as that of the ions because chargeexchange dominates over ionization. The DEGAS tables for densitydependent ionization and radiation are used. The gas diffusion flux is limited to the ion thermal flux. We can also separate the Franck-Condon and charge-exchange neutrals into two species. We have just begun to assess the effect of this description for a linear divertor simulator. While we initially did not expect much difference between the two descriptions, we have found a sensitivity to particle fueling to be discussed later for the one-species model that may yield a significant difference. The diffusive gas model has been

1 D.A. Knoll, A.K. Prinja, and R.B. Campbell, J. Comp. Phys. 104, 418 (1993).

2 T.D. Rognlien, J.L. Milovich, M.E. Rensink, and G.D. Porter, J. Nucl. Mats. 196-198, 347 (1992).

3 B.J. Braams, NET Report Nr. 68, (1987). 
benchmarked with the neutral Monte Carlo code DEGAS for a TPX calculation with satisfactory results. These neutral gas models are a departure from the original B2 analytic gas model, although there are a variety of neutral models used in newer $\mathrm{B} 2$ versions.

\subsection{Geometry, Sources, and Parameters for the Calculations}

In the calculations that follow, we use cylindrical geometry where the lefthand boundary corresponds to the midplane and the right-hand boundary the divertor plate. A uniform axial magnetic field is assumed. The radial boundary is taken at $5 \mathrm{~cm}$ and the half-length from the midplane to the divertor plate is $13 \mathrm{~m}$. The boundary conditions at the midplane use reflection symmetry; some of the calculations have a limiter at the midplane beyond $4.25 \mathrm{~cm}$ where divertor plate boundary conditions are used. At the divertor plate(s), the ion parallel velocity reaches the ion acoustic speed, and a neutral flux of $R$ times the ion flux flows back into the plasma.

Particles and power are injected into the plasma by fixed volume sources near the midplane with (shifted) Gaussian profiles in the axial and radial directions. For simplicity, we have taken the ion power profile to be uniform radially, and having a Gaussian scale length of $5 \mathrm{~m}$ axially. An electron power source is available, but has not been used; the electrons are collisionally heated by the ions. The ion particle source has the same axial profile as the ion power, but we consider two cases of the radial profile. One is termed central fueling where the Gaussian is centered on axis with a $3 \mathrm{~cm}$ scale length. The second, termed edge fueling to simulate gas-box fueling with a limiter, has its peak are $4 \mathrm{~cm}$ and a scale length of $2 \mathrm{~cm}$.

The mesh used for the calculations is nonuniform, with most of the resolution near the end plate to better resolve the recycling zone where the gas is ionized. Because the midplane limiter also needs a fine mesh near the limiter, we use two different meshes depending on whether or not the limiter is included. The two meshes are shown in Fig. 5.1, both of which are $32 \times 16$ cells for the axial and radial directions, respectively.

In the calculations that follow, several parameters are held constant to aid in interpretation. The ion power is $2 \mathrm{MW}$ for the half of the device simulated. The anomalous radial diffusion coefficients are all set to $1 \mathrm{~m}^{2} / \mathrm{s}$, and there is no pinch term. The effective charge $Z_{\text {eff }}$ is taken as unity, so no enhanced electron-ion coupling occurs. Finally, the particle source is adjusted to keep the peak midplane density to be approximately $4 \times 10^{19} \mathrm{~m}^{-3}$. 

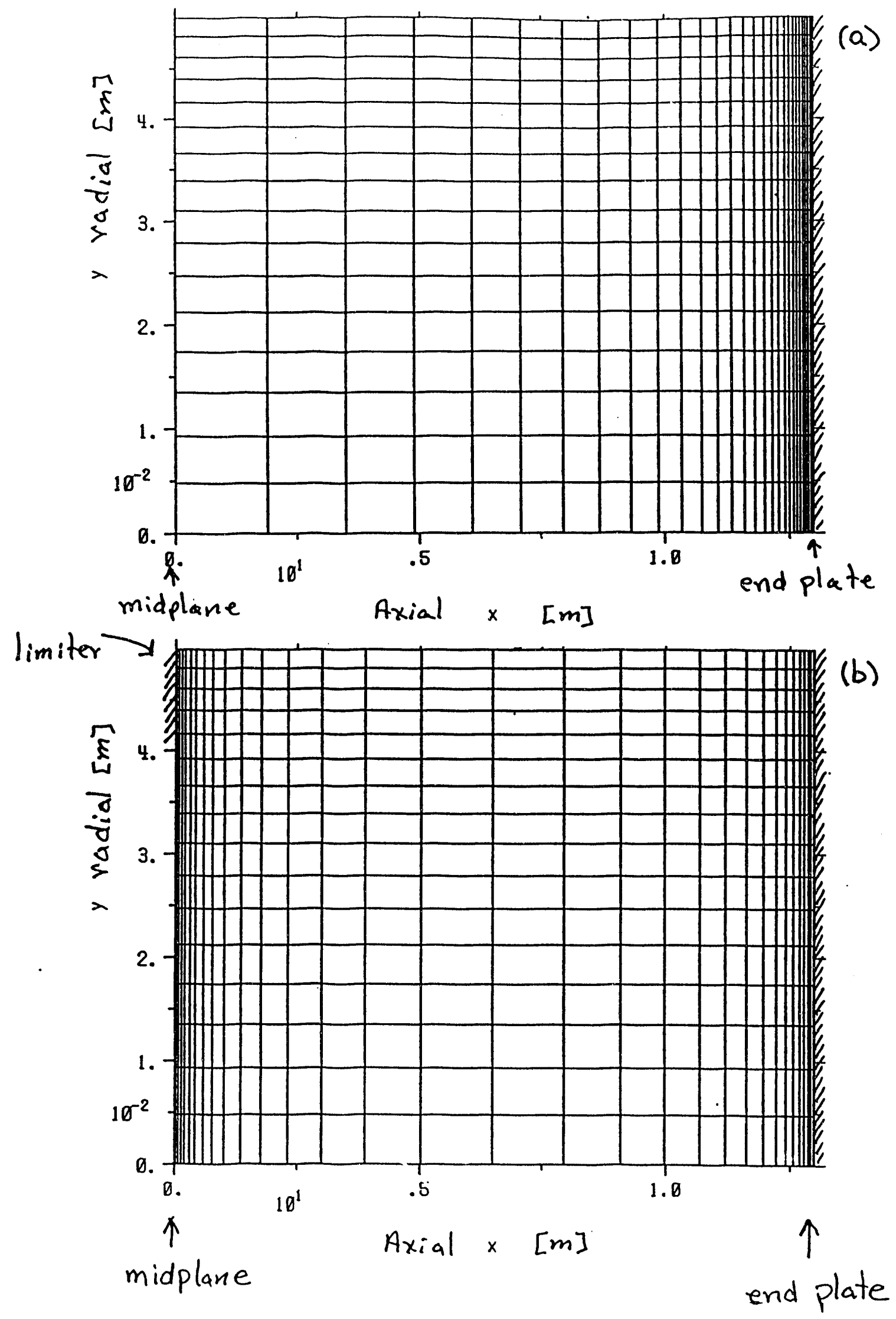

Fig. 5.1 The grid used for the two base cases: (a) central particle fueling with no limiter, and (b) edge particle fueling with a radial limiter. 


\subsection{Results for Central Fueling}

For the base case, we use a recycling coefficient of $R=0.9$ and a volume particle source to $1 \mathrm{kA}$ to maintain a midplane density of about $4 \times 10^{19} \mathrm{~m}^{-3}$. These parameters are chosen to model the simulation performed by the ICEPIC code in Sec. 2. The resulting contour plots are shown in Fig. 5.2 where the axes are in cell index number, so the region near the end plate is expanded. In addition, the neutral gas diffusion coefficient for the axial direction is increased in the vicinity of the end plate to give sufficient resolution of the gas; we believe this does not appreciably effect the properties of the plasma except that the recycling zone is extended in the axial direction. The edge temperature is taken to be $2 \mathrm{eV}$ and radial gradient of the ion density is zero at the wall; the latter condition will be relaxed in the next section. A peak electron temperature of a little more that $50 \mathrm{eV}$ is sustained by collisional heating from the ions. This is somewhat hotter than the ICEPIC calculations for the corresponding $25 \mathrm{~kW} / \mathrm{cm}^{2}$ at the true electron mass. One reason for this difference is that here the hot ions are accelerated axially by the $\mu \nabla B$ force, which decreases the time that the hot ions have to heat the electrons in ICEPIC.

Note the end plate has a hot, low density core and a cold, high density outer region. These features can be seen more clearly from plots of the radial profiles at the end wall shown in Fig. 5.3. Note that the heat flux is carried mostly in the ion channel which reaches $260 \mathrm{MW} / \mathrm{m}^{2}$ on axis. The electron heat flux is $70 \mathrm{MW} / \mathrm{m}^{2}$. The power that flows to the radial wall is about 1 MW, which is determined by taking a low boundary temperature and having a significant edge density from the zero radial flux boundary condition. Some of this power will flow to a radial limiter that we model in the Sec. 5.5.

The second case considered has $R=0.99$, and a volume particle source of $0.22 \mathrm{kA}$ to maintain the midplane density. The end plate profiles are shown in Fig. 5.4. The density is more filled in on axis, and the ion and electron temperatures are both below $15 \mathrm{eV}$, indicating the high recycling regime. Furthermore, the peak heat flux is now in the electron channel and the total peak heat flux is reduced by about $30 \%$.

The third case is a slight variation of the second to show the potential sensitivity of the equilibrium. By reducing the particle source $10 \%$ to $0.20 \mathrm{kA}$ with $R=0.99$, the column develops a small, hot core region again as shown in Fig. 5.5. A decrease in the power injected into the ions reduces the hot core. The point here is that the end plate conditions can be sensitive functions of the input parameters in the expected operating range. These different states may exist in the ITER divertor as well, and understanding them will be important. In beginning to modeling these cases with the two-species gas 
$50-3000 \cdot 10110+3000^{\circ}$ \& $519 H$

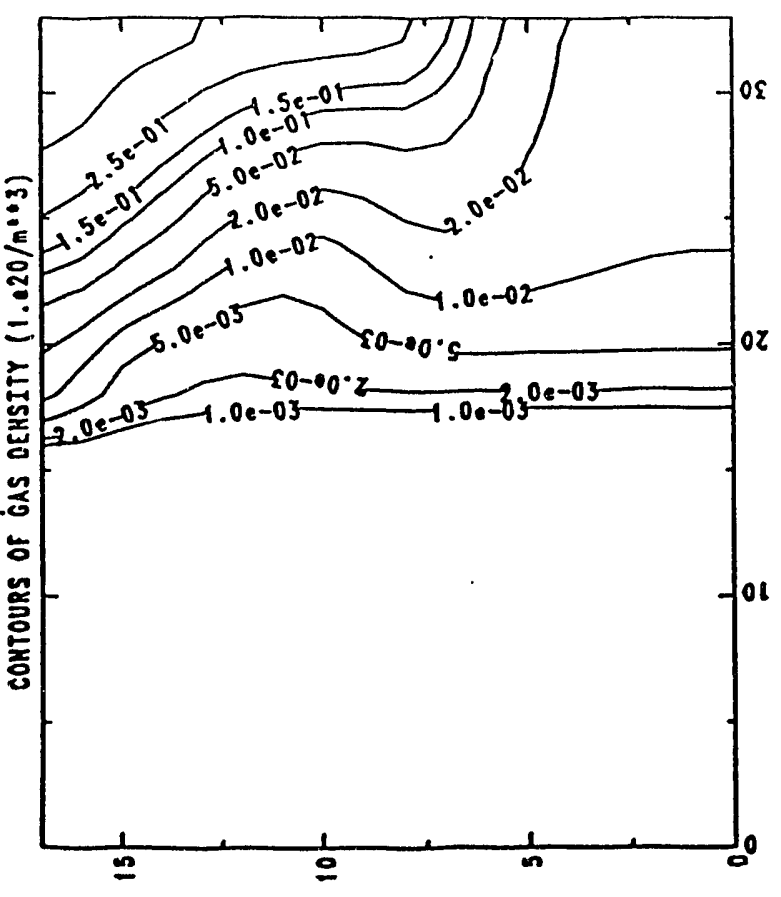

xJOKI 7าวง TVIOY

$20-3000 \% 0110+3000^{\circ} \&$ SLOH

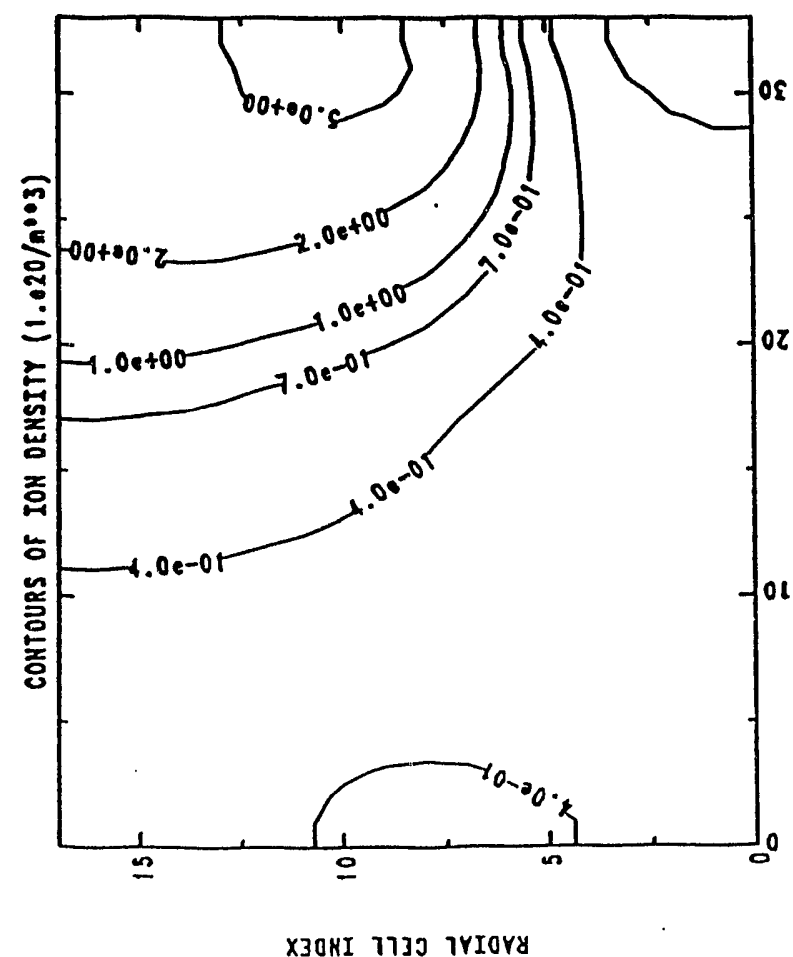

$20+3000^{\circ}$ S $0100+3000^{\circ} / \mathrm{S} 19 H$

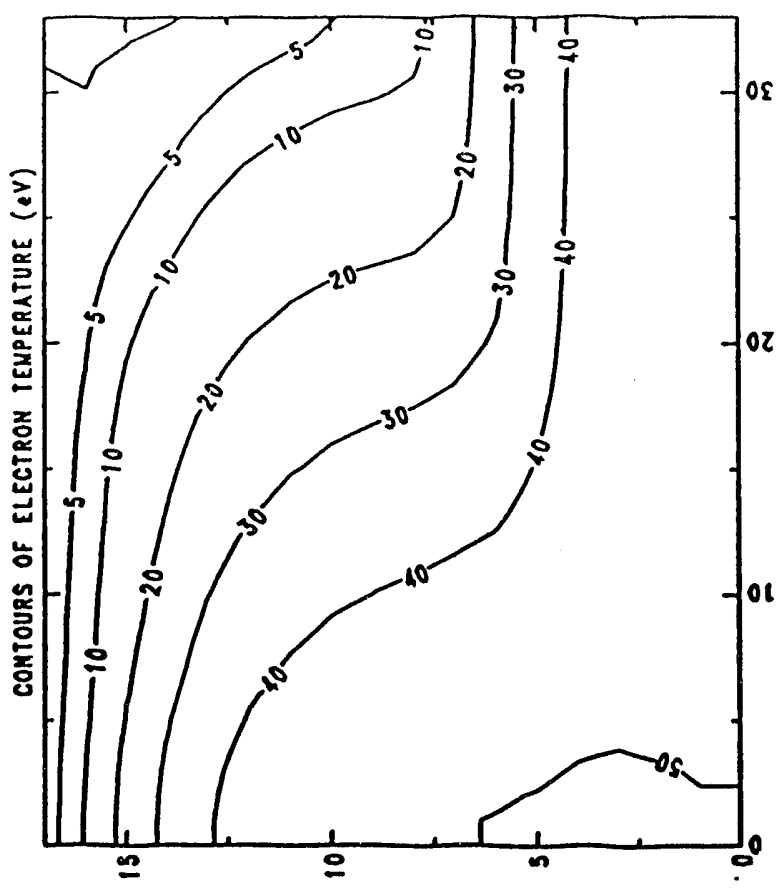

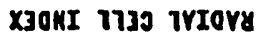

$20+3000^{\circ} \subseteq 0100+3000 \% 1$ SLOH

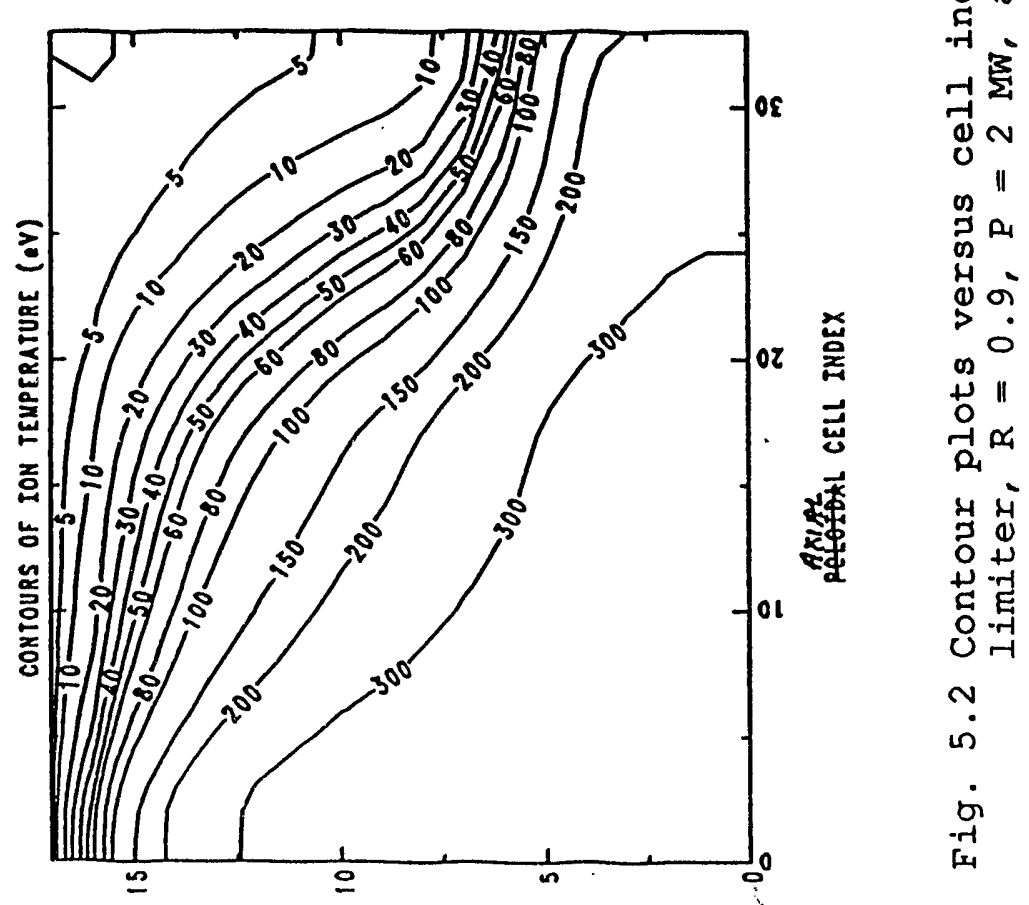

X3ONI 1730 TYIOYY
울

$\underset{-4}{-4}$

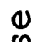

ర్ల

号

号

岁安

댐

of $\mathrm{H}$

ฮั

ન્નુ

$\rightarrow$

岱步

"I

कू م

के

0

$\operatorname{lin}^{\infty}$

i.

है

걷

O.न

N

$\underset{r}{0}$ 


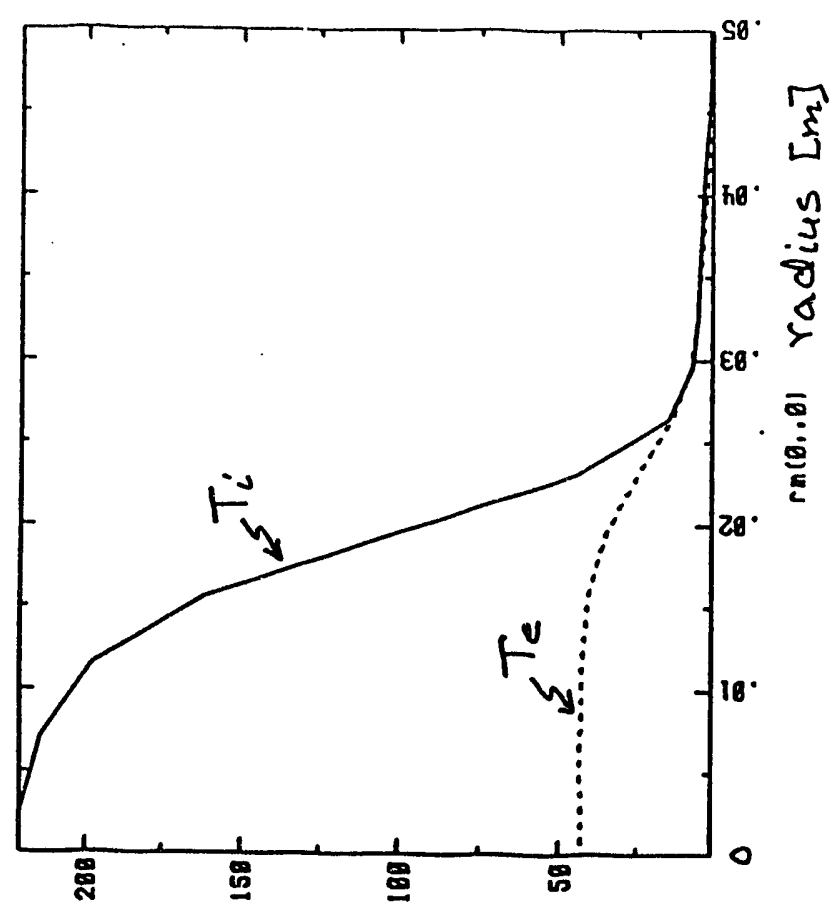

5
5
5
5
8
8
5
5
5
5

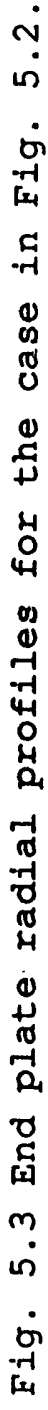

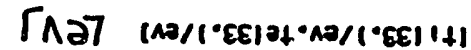

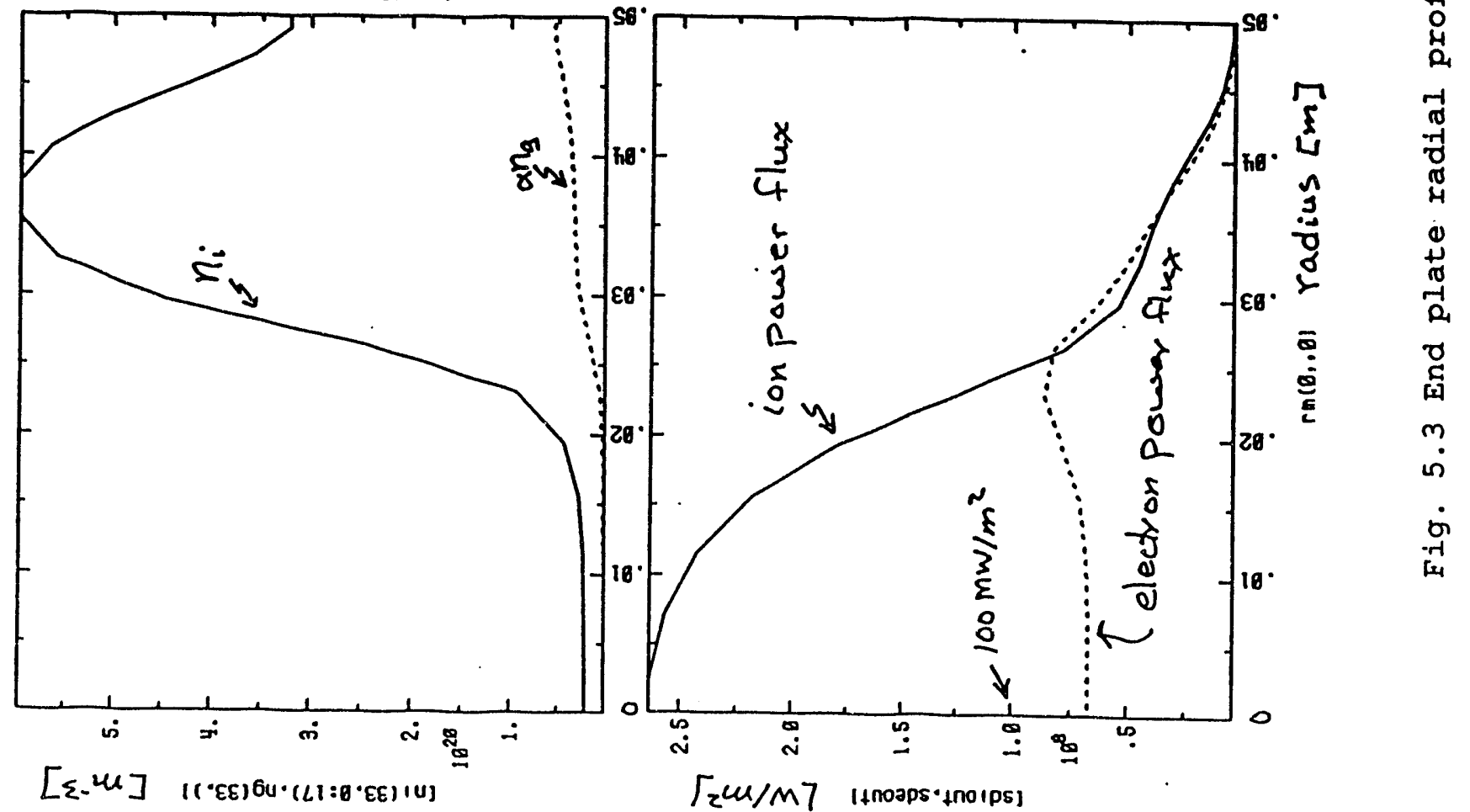




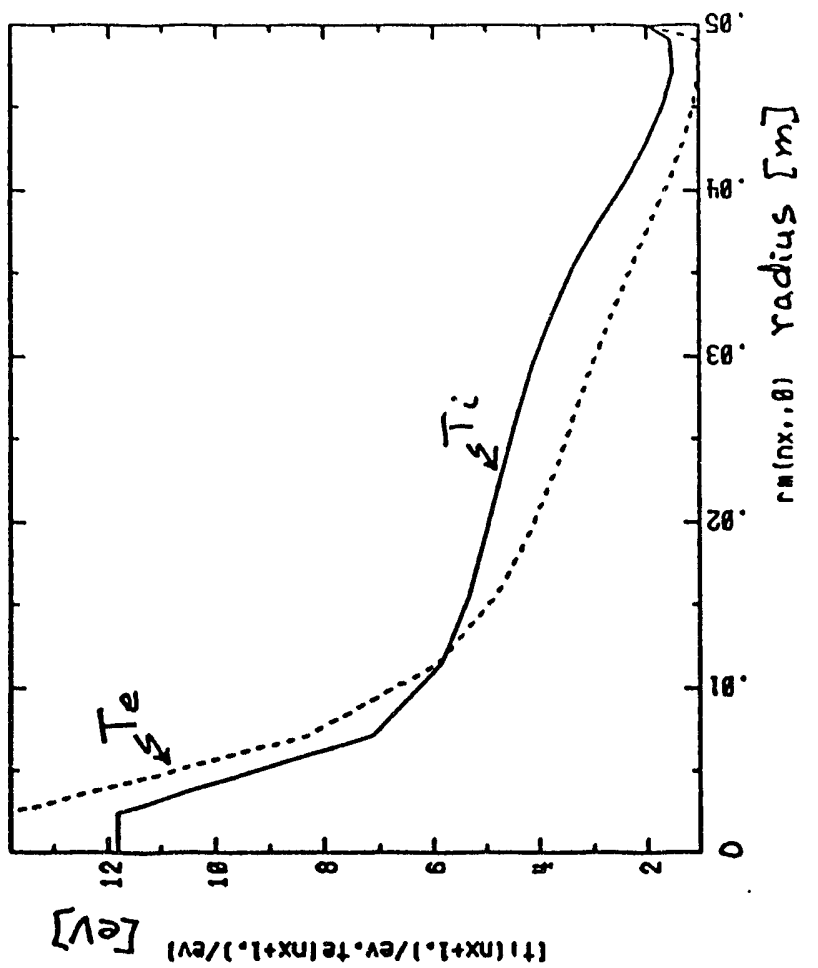

मे
0
-1
-1
-7
-1

옹

兵

a

0

0

D

岱

بِ

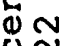

40
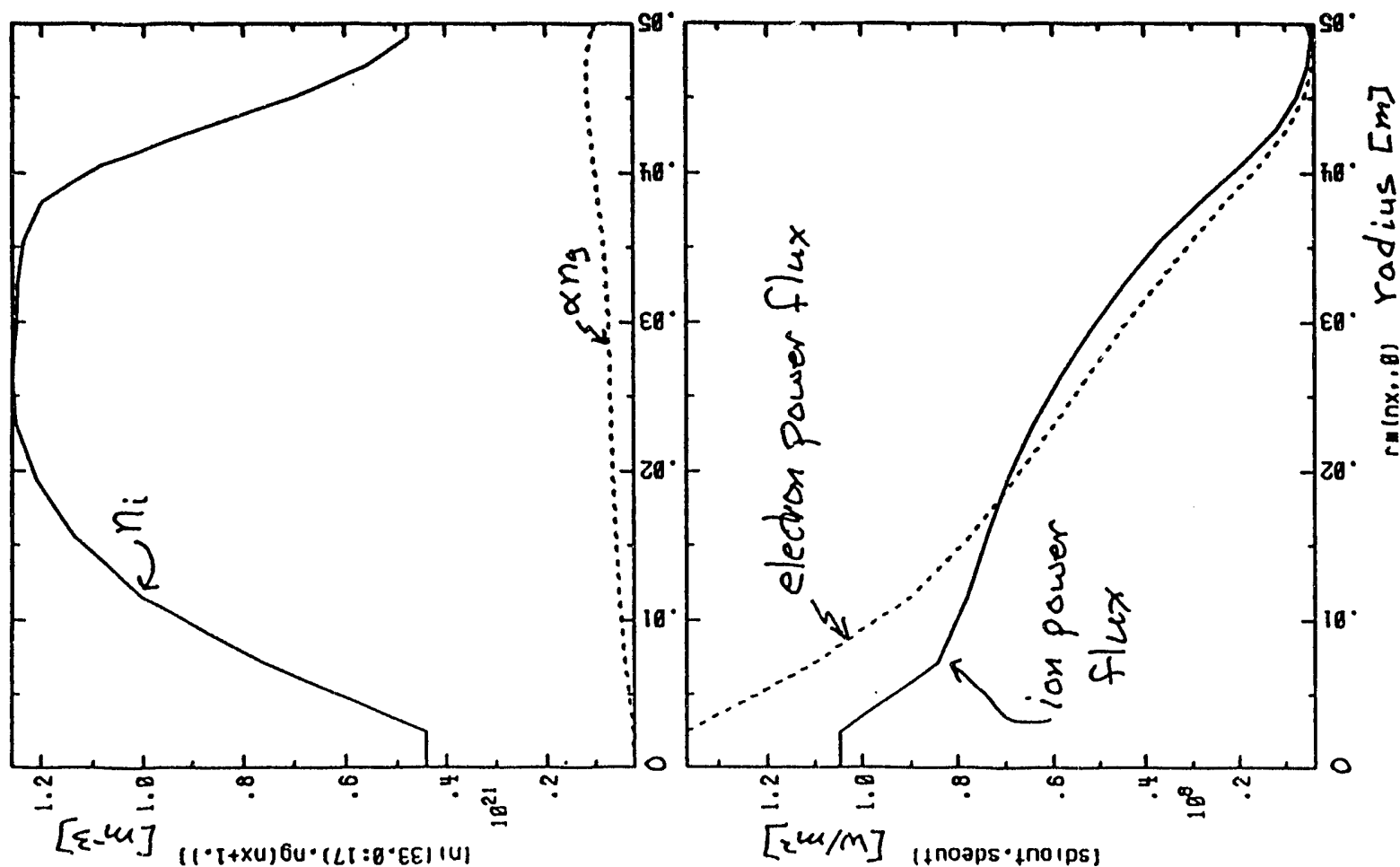

or

거엉

पै

मू

กั

नु ㅅ

兵

:

त.

'大

쇠

in

क्ष 


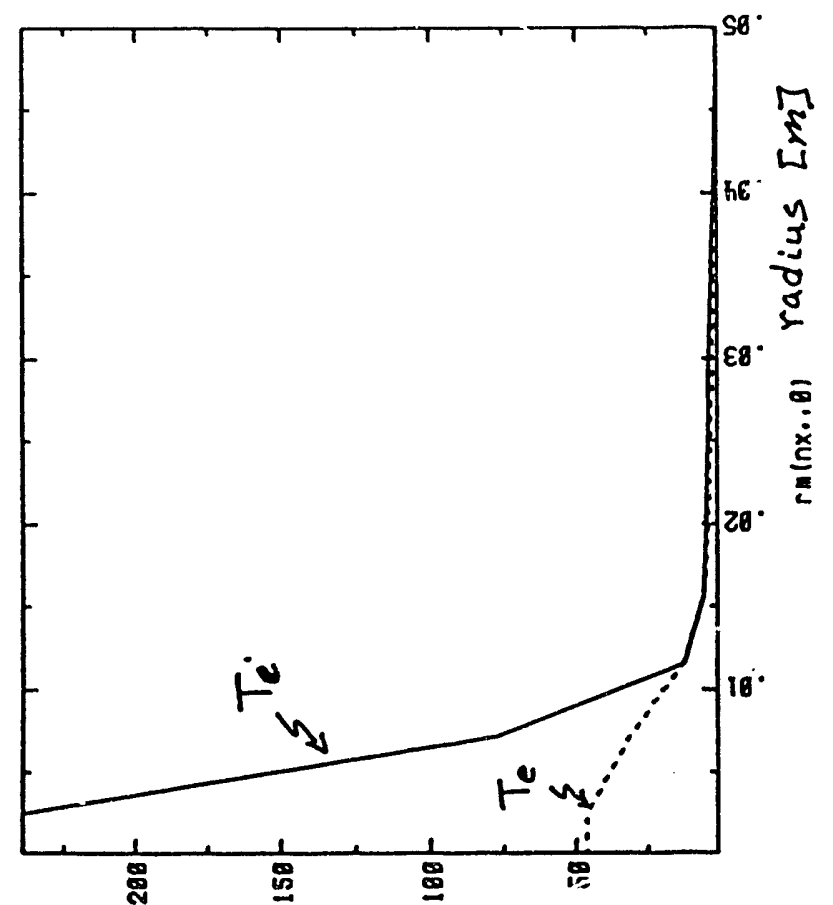

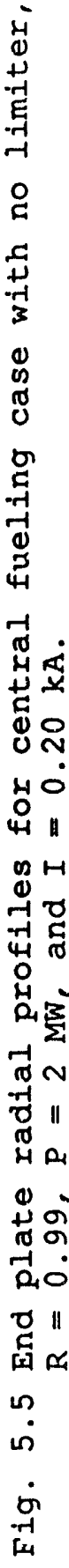

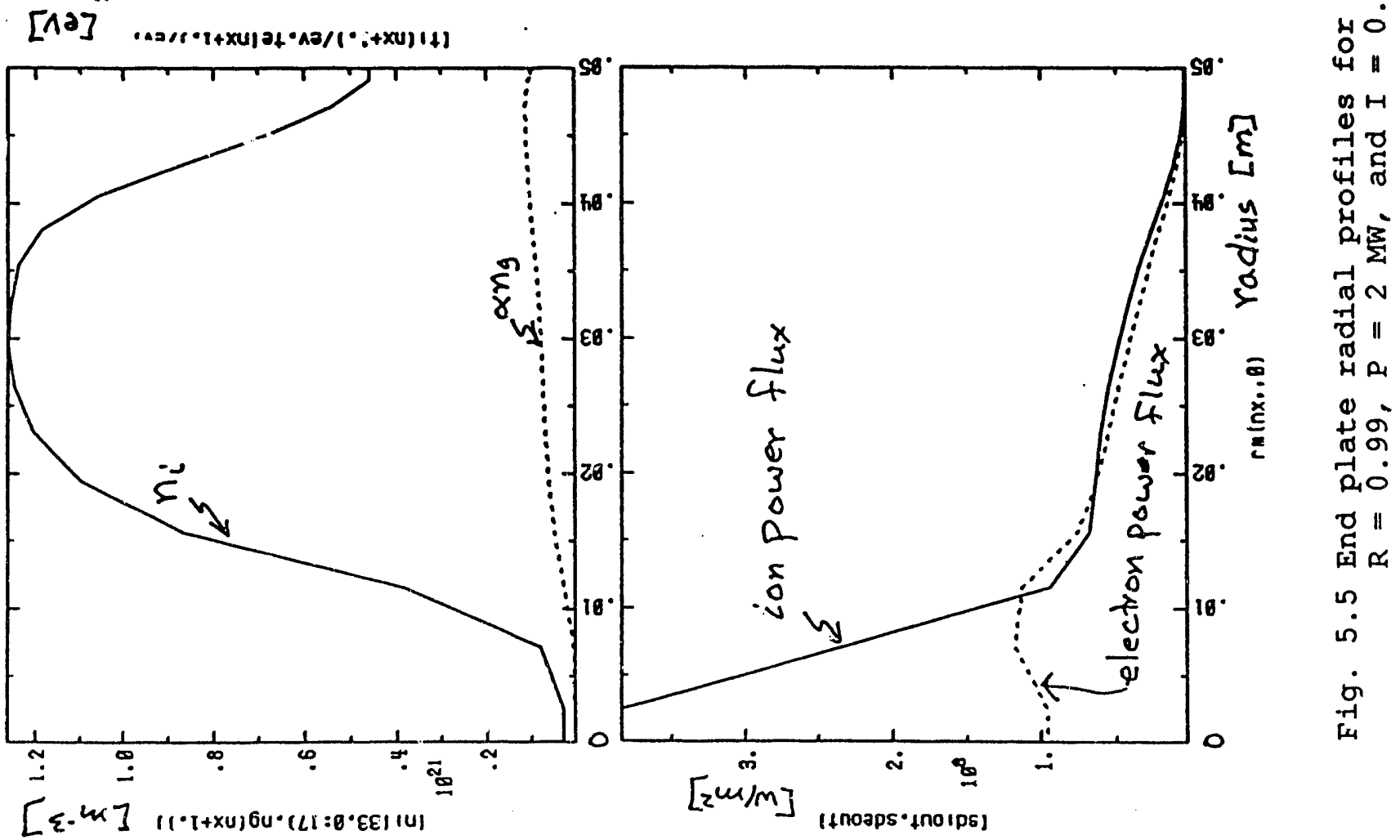


description, we have also encountered strong sensitivities that we are working to understand.

\subsection{Results for Edge Fueling with a Radial Limiter}

For these simulations, we move the midplane particle source to $r=4 \mathrm{~cm}$ with a Gaussian scale length of $2 \mathrm{~cm}$ to model fueling from a gas box. A limiter is also used for $r>4.25 \mathrm{~cm}$ at the midplane to detci inine the power flow to it. Both the particle and power sources are zero on the limited field lines $(r>4.25 \mathrm{~cm})$, so any current and power reaching its surface comes from cross-field transport. The radial boundary condition on the ion density is now a fixed density of $6 \times 10^{18} \mathrm{~m}^{-3}$. To compare with the base case, the recycling coefficient is $R=0.9$.

A set of contour plots of the solutions are shown in Fig. 5.6. The midplane ion density is about $3.5 \times 10^{19} \mathrm{~m}^{-3}$, and there is a reversed flow (not shown) of plasma to the limiter; note the presence of neutral gas from the limiter surface. The ion temperature reaches over $700 \mathrm{eV}$, and the ion density shows a stronger minimum on axis owing to the edge fueling. End plate profiles and the limiter heat flux profiles are shown in Fig. 5.7. While the total power to the limiter is about $8 \%$ of that to the end plate, the peak heat flux to the limiter is $80 \mathrm{MW} / \mathrm{m}^{2}$; this would certainly require tilting the limiter to increase its surface area. How this heat load scales with parameters has not yet been determined. Another comparison of the central and edge fueling cases is shown in Fig. 5.8 where the ion densities are three axial locations are shown. The unlabeled dotted profile corresponds to $z=6.5 \mathrm{~m}$ for the edge fueling case and $z=11.8 \mathrm{~m}$ for the central fueling case.

The peak energy flow in the hot core region will be reduced if the RF power absorption is peaked more on the edge; recall that here we take a radially uniform power source. On the other hand, an edge localized power source will enhance the undesirable heat flux to the limiter. There is also a heat flux to the radial wall, which, for the case considered here, is about 0.5 MW. It is lower than in the central fueling case because the edge density is lower.

\subsection{Summary}

- The collisional coupling from the ions to the electrons results in $T_{e}$ of about $50 \mathrm{eV}$ for $2 \mathrm{MW}$ into the ions and an ion density of $4 \times 10^{19} \mathrm{~m}^{-3}$. This is somewhat greater than a comparable case from ICEPIC, likely because of the absence of the $\mu \nabla B$ force to expel hot ions in UEDGE. 


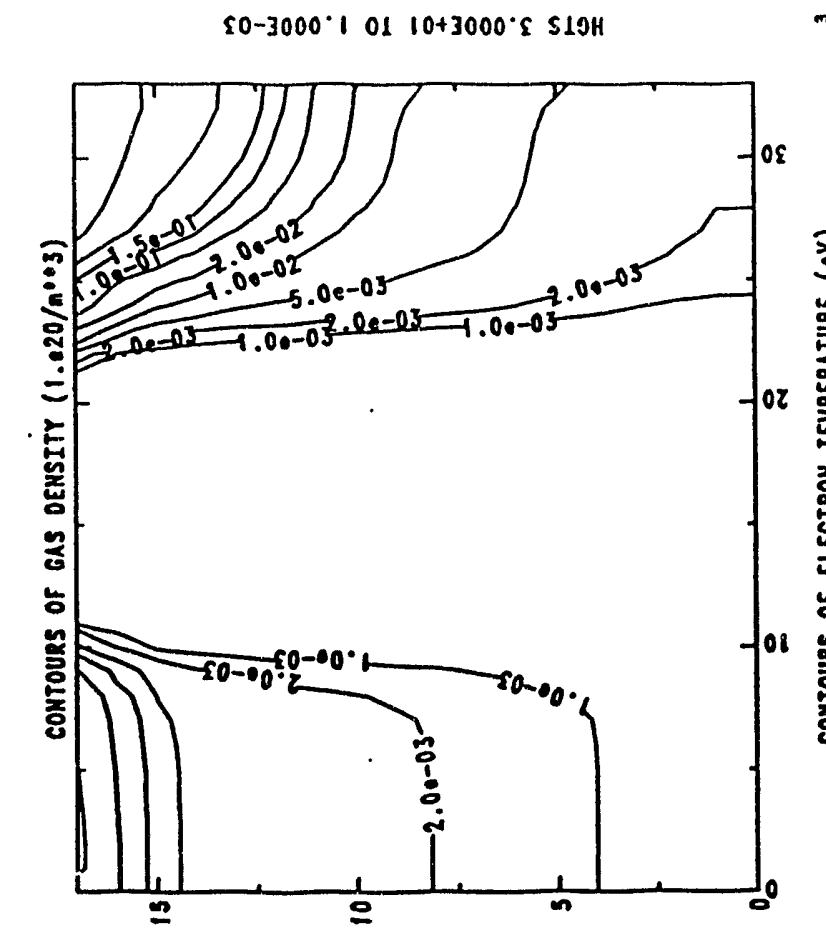

m $20+3000^{\circ} \mathrm{SOL} 00+3000 \div \mathrm{S} 19 \mathrm{H}$
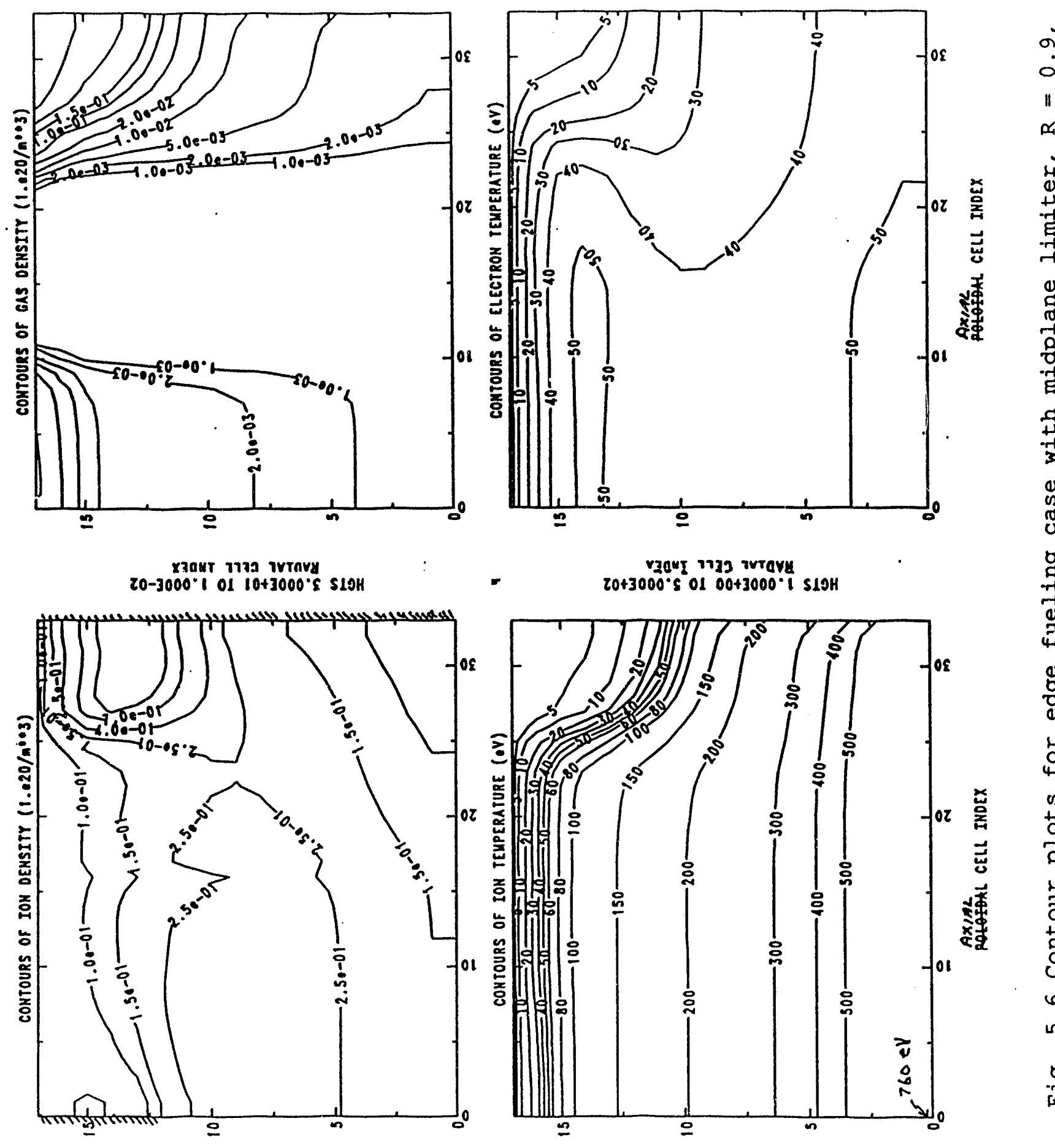

X3anI 17נכ iricry
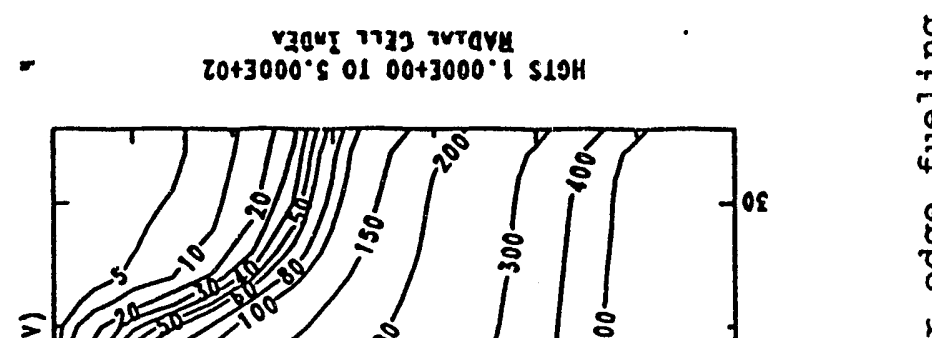

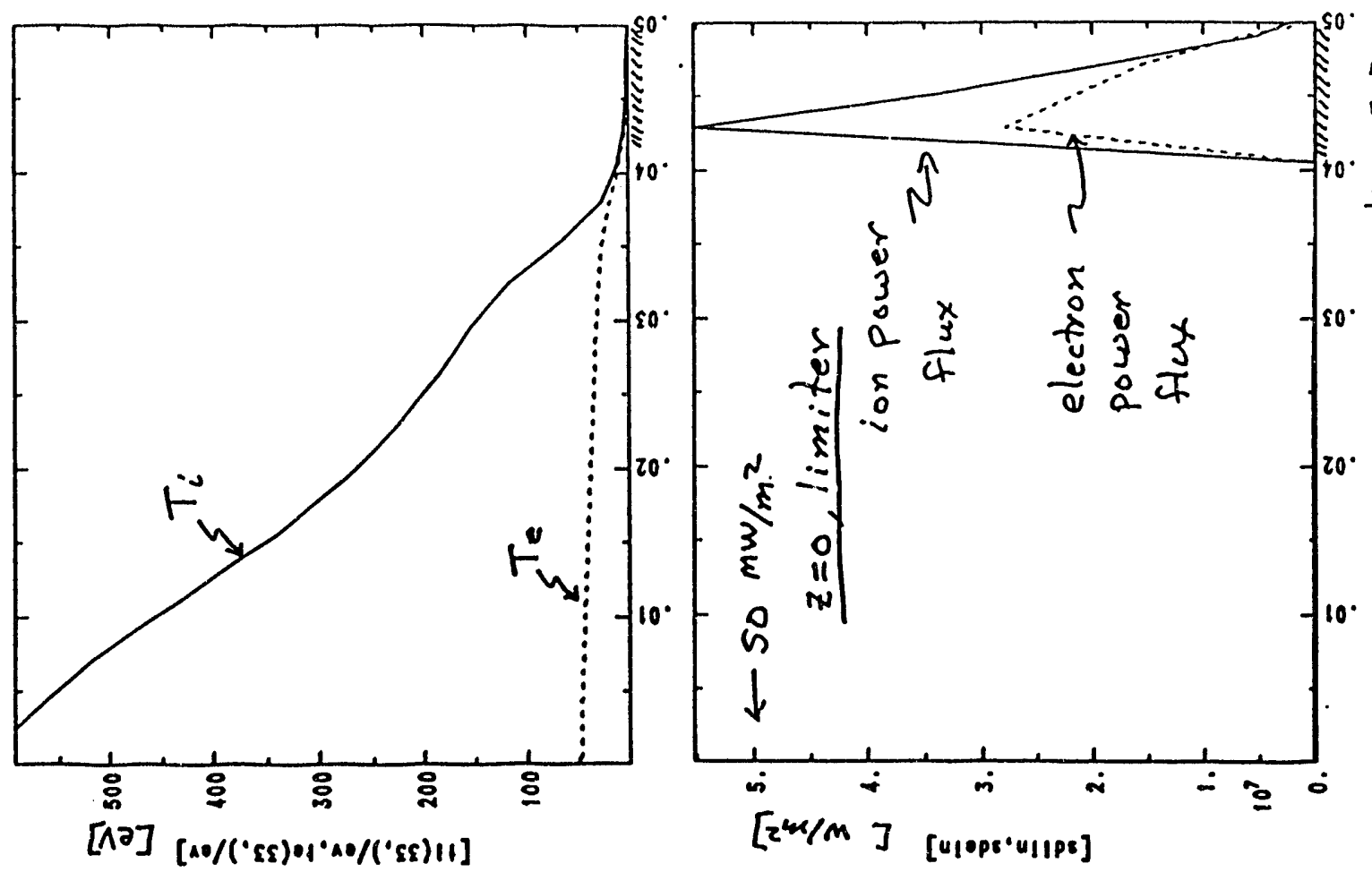

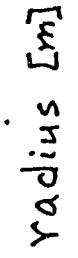

$\dot{0}$
$-\quad \dot{ }$

0
$\dot{0}$
$\dot{0}$
0
-1
4
.
-1
0
0
0
0
4
0
4
0
0
-1
4
4
0
4
0
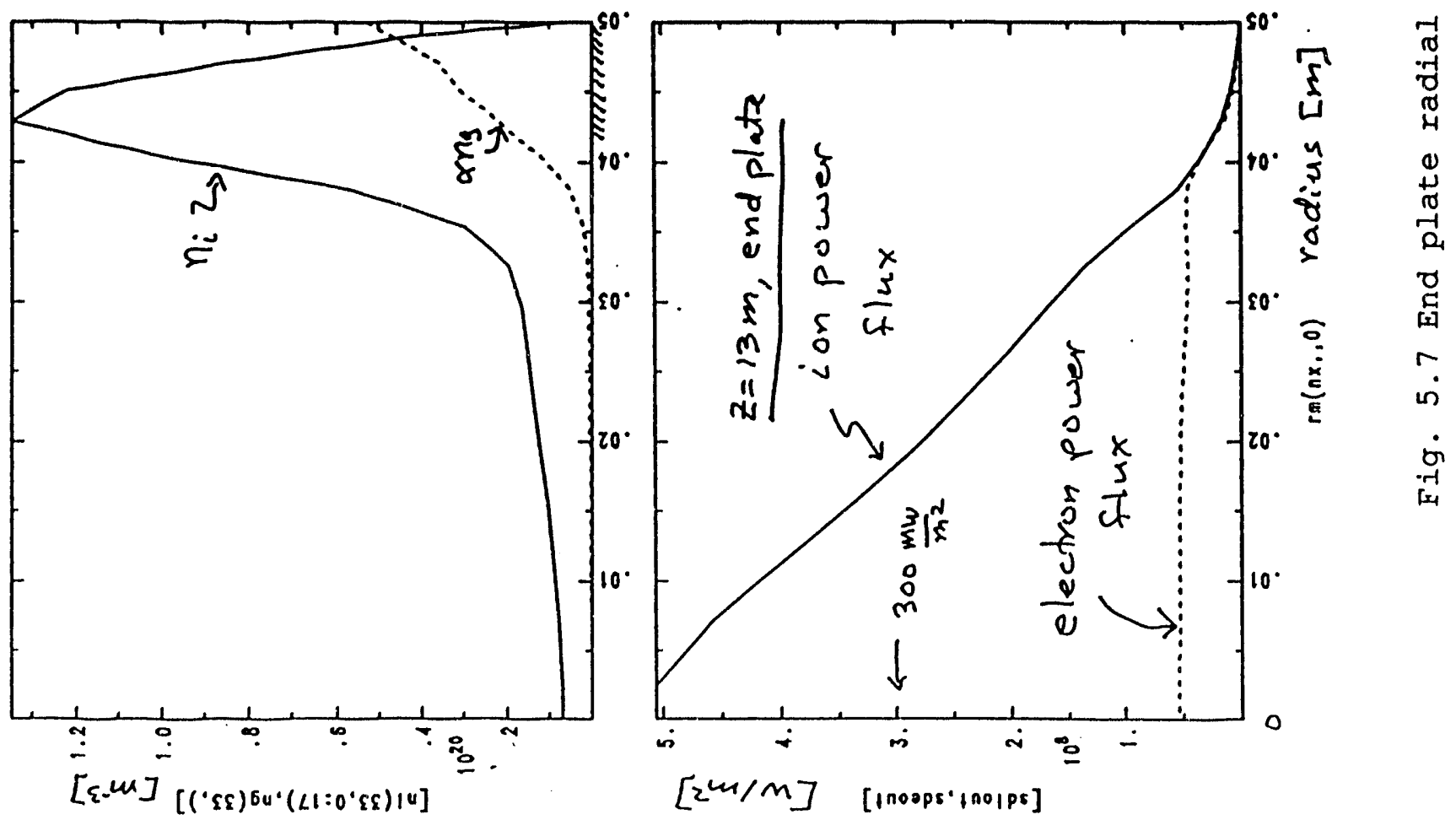

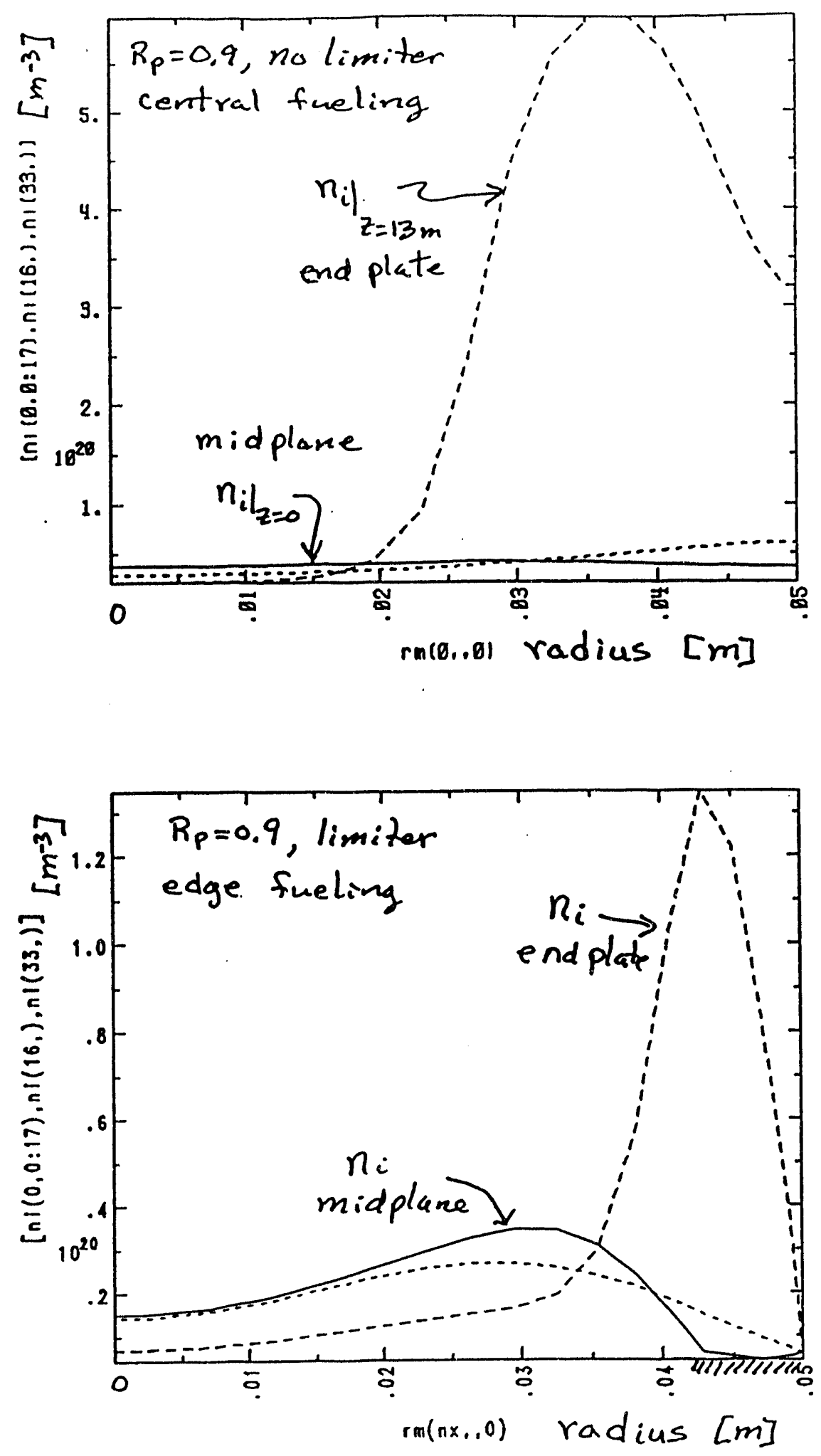

Fig. 5.8 Radial density profiles at several axial locations for the central fueling case and the edge fueling case, both with $R=0.9$. 
- For central fueling, inner core region is in low recycling mode unless $R>$ 0.99. Outer radial region is in high recycling regime.

- For edge gas-box-like fueling, it is more difficult to get the core region into the high recycling mode.

- Total power to radial midplane limiter is about $8 \%$ of that to end plate, and the peak heat flux is $80 \mathrm{MW} / \mathrm{m}^{2}$.

- $\quad$ Radial power loss varies from $20-50 \%$ of input power, depending on boundary conditions, fueling location, and anomalous diffusion coefficients. 


\section{Review of ICRF Heating Experiments in Linear Devices}

\subsection{Power accountability in ICRF heated mirrors}

\section{A. W. Molvik}

Power balance studies with ICRF, Ion Cyclotron Range of Frequencies, heating have been conducted in a number of mirror machines. Two studies that were especially thorough are discussed here.

Experiments were conducted with a slot antenna in the TARA tandem mirror. ${ }^{1}$ A slot antenna is similar to one section of the antenna proposed for IDEAL making these results particularly relevant. Golovato and coworkers were able to account for $65-73 \%$ of the launched ICRF power. They found that $\sim 50 \%$ heated the ions, and $\sim 25 \%$ heated the electrons. Only about $5 \%$ of the ICRF power heated the electrons through drag. About $20 \%$ of the ICRF power heated the electrons directly through Landau damping.

About $30 \%$ of the ICRF power in TARA was unaccounted for. Golovato, et al. speculated that the cause could he radial losses at gas box, based on $50 \%$ of the gas fueling not showing up as end-losses. Another possible cause could be heating of scrape-off-layer plasma which would then dump the power onto limiters.

Experiments were conducted with two independently powered doublehalf-turn ICRF antennas in TMX-U, the Tandem Mirror Experiment Upgrade. ${ }^{2,3}$ Falabella and coworkers were able to account for $88 \pm 16 \%$ of the ICRF power on 4 sets of shots that were analyzed in detail. The ion energy distribution was measured with a radial retarding potential energy analyzer (Termed the REA for Radial Energy Analyzer, Fig. 1) inserted into the plasma. The accuracy of the measurements of temperature, density, and plasma potential was corroborated by comparison with diamagnetism, line density, end-loss energy(where appropriate), and the heavy-ion beam probe plasma potential diagnostic. The comparison with the end-loss ion temperature is shown in Fig. 2, good agreement is found when the ICRH (Ion Cyclotron Resonance Heating) is off and the ions are expected to be isotropic. When the ICRH is on, the ions in the central cell that are measured by the REA are heated to perpendicular temperatures exceeding the parallel temperatures measured at the ends of TMX-U. The perpendicular energy can be more

\footnotetext{
1 S. Golovato, et al., "Plasma Production and Heating by ICRF in TARA," Phys. Fluids 31, 3744 (1988).

2 S. Falabella, "Measurements of radial profiles of ion cyclotron resonance heating on the Tandem Mirror Experiment Upgrade," Ph.D. Thesis UCRL-53870, 1988.

3 S. Falabella and A. W. Molvik, "Ion temperature measurements on TMX-U using a gridless electrostatic analyzer," Rev. Sci. Instr. 61, 1892 (1990).
} 
directly compared with the diamagnetism, Fig. 3. The ion pressure profile is computed from the ion density and temperature obtained during a radial scan of the REA. Good agreement with diamagnetism is obtained when the electron pressure is included. Similar good agreement is found with potential measurements.

The result was that the plasma core was heated, Fig. 4,5, but somewhat less than the plasma edge, inside the limiter, Fig. 4,5, both compared with ICRH off, Fig. 6. The heating profiles were compared with those calculated with the ANTENA code ${ }^{4}$ and the GARFIELD code. ${ }^{5}$ No convincing correlations were found, which was attributed to the axially uniform magnetic field (precluding magnetic beach ion cyclotron absorption) and the collisional damping model in ANTENA. GARFIELD includes the axial variation of the magnetic field, therefore shows the proper wave behavior and absorption near the cyclotron resonance, but still has a simplified collisional heating model.

The power balance summarized above did not require direct electron or scrape-off-layer heating in order to account for the power input compared to the measured power losses. The following five ion power loss mechanisms were evaluated 2 and are shown in Figs 4-6: (1) Charge-exchange loss rates were obtained with the ion energies measured as a function of radius by the REA and the current was computed by scaling the charge-exchange rate from the $\mathrm{H}_{\alpha}$ measurement of the ionization rate for the measured ion and electron temperatures. (2) End-loss temperatures as a function of radius were obtained from the REA, using a scaling factor of 0.4 to 1.0 from the ratio of end-loss energies on axis to the REA also on axis. The plasma potential as a function of radius was also measured by the REA and increases the energy of ions reaching a grounded end wall. The end-loss current as a function of radius was obtained from the Faraday cup array. (3) Electron drag was computed assuming a 2 step profile, $\mathrm{T}_{\mathrm{e}}=40 \mathrm{eV}$ near the axis as measured by Thomson scattering, and $\mathrm{T}_{\mathrm{e}}=20 \mathrm{eV}$ near the edge as measured by a Langmuir probe. The ion energy and density came from the REA. (4) Radial loss currents were determined from the difference between the $\mathrm{H}_{\alpha}$ measurement of the fueling rate and the Faraday cup measurement of the end-loss rate. The loss energy is the sum of the potential and ion energy, both measured by the REA. (5) Excitation losses were taken to be the product of the ionization current times $30 \mathrm{eV}$ per ion.

4 B. McVey, "ICRF Antenna Coupling Theory for a Cylindrically Stratified Plasma," MIT Report PFC/RR-84-13, and "ANTENA User Guide," MIT Report PFC/RR-84-13, Cambridge (1984).

5 M. W. Phillips, A. M. M. Todd, Computer Physics Communication 40, 65 (1986). 


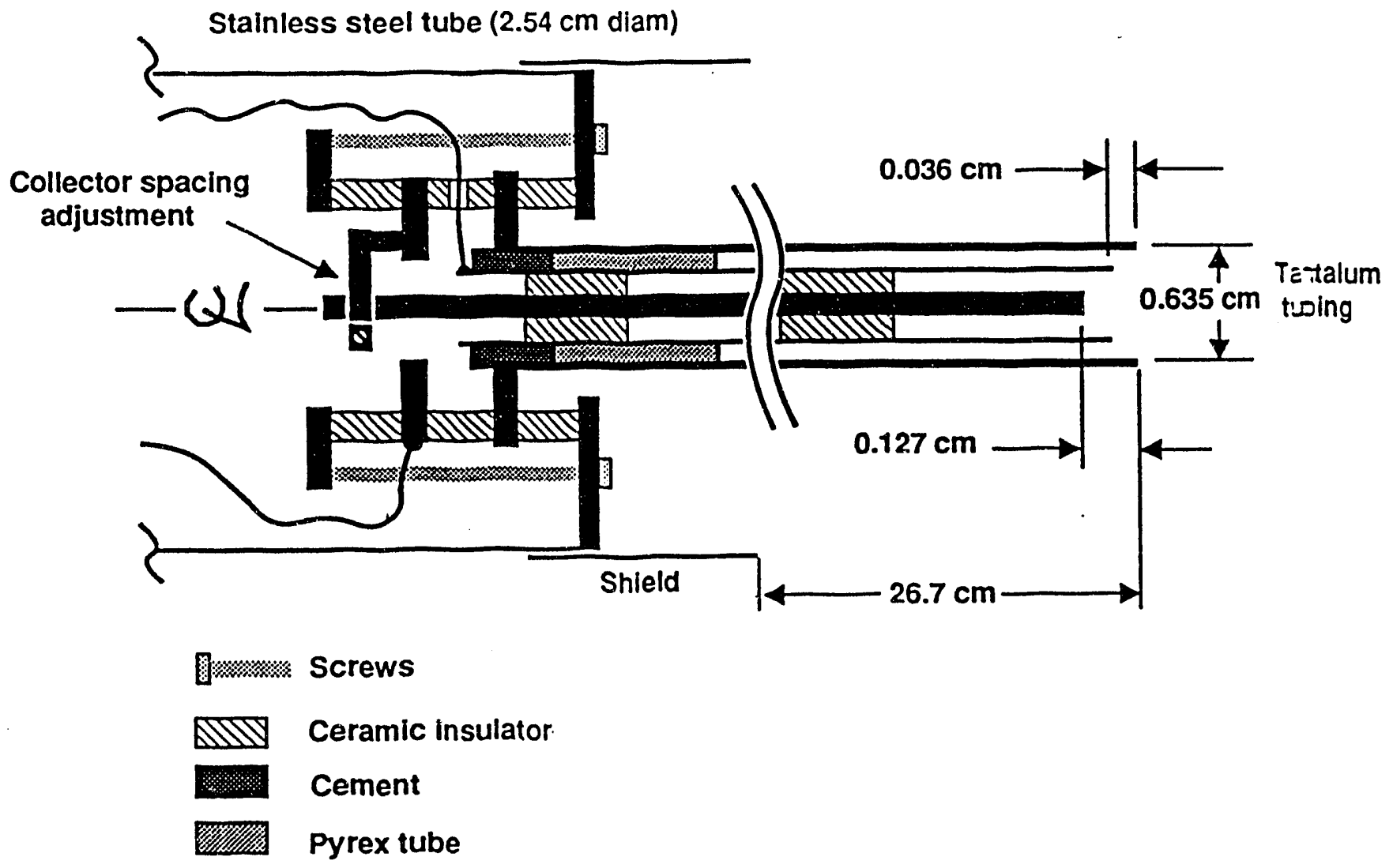

Fig. 6.1-1 REA tip detail. 


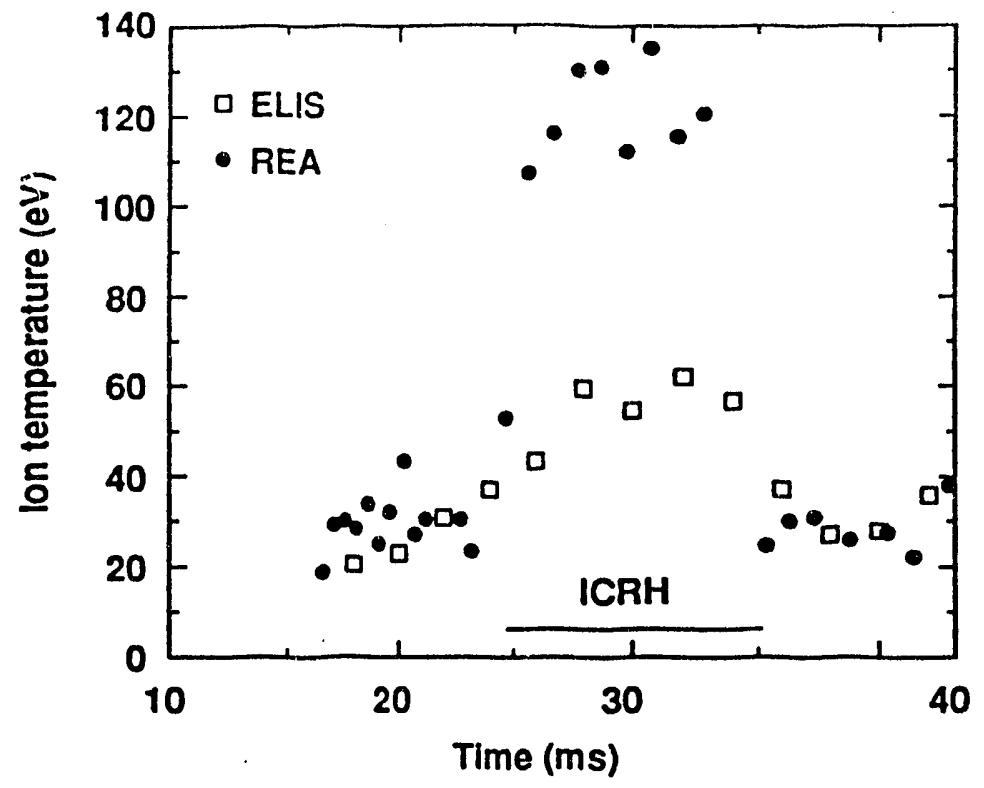

Fig. 6.1-2 Comparison of ion temperature measured by the REA and end-loss ion spectrometer.

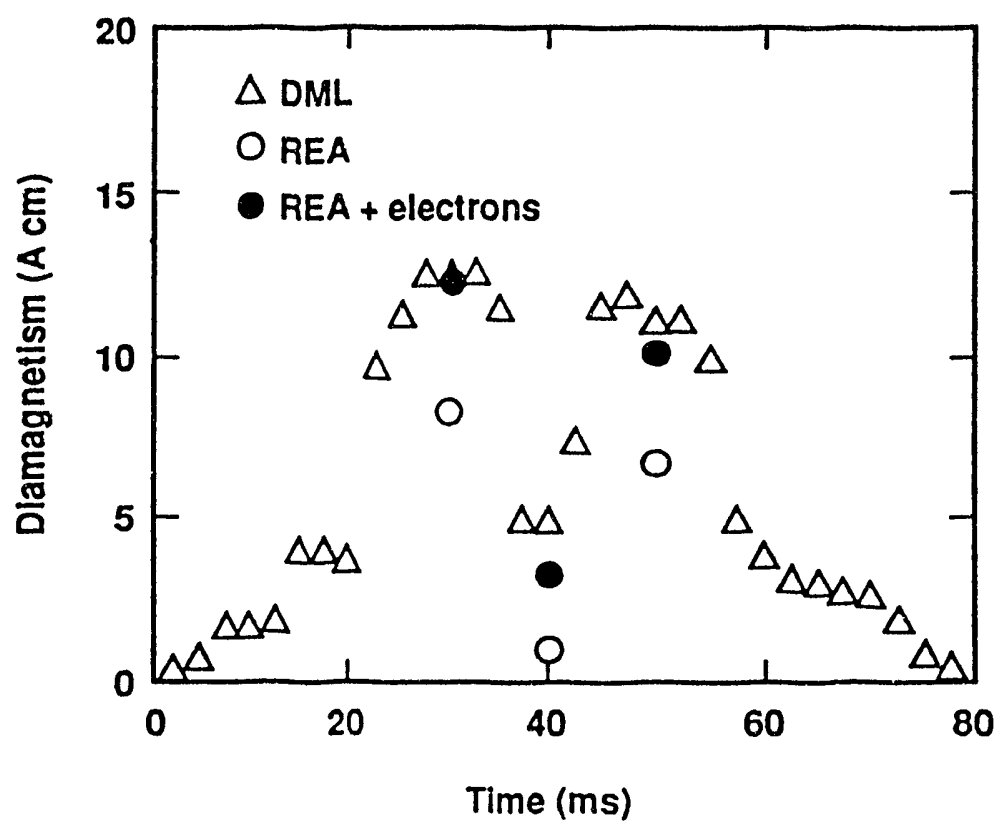

Fig. 6.1-3 Measurements of stored plasma energy by the REA and by diamagnetic loop show good agreement. 


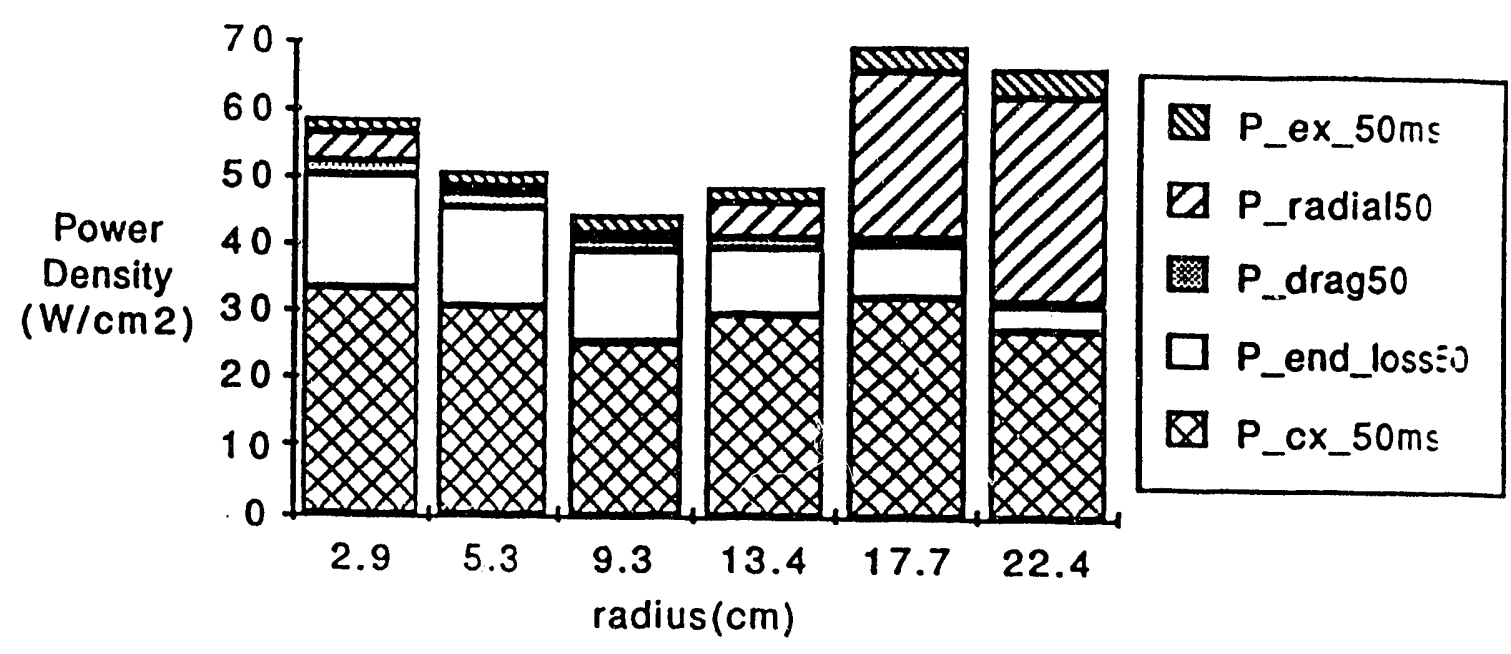

Fig. 6.1-4 Radial Profile of Power Loss Terms for the East Antenna, for Sian Number 5 


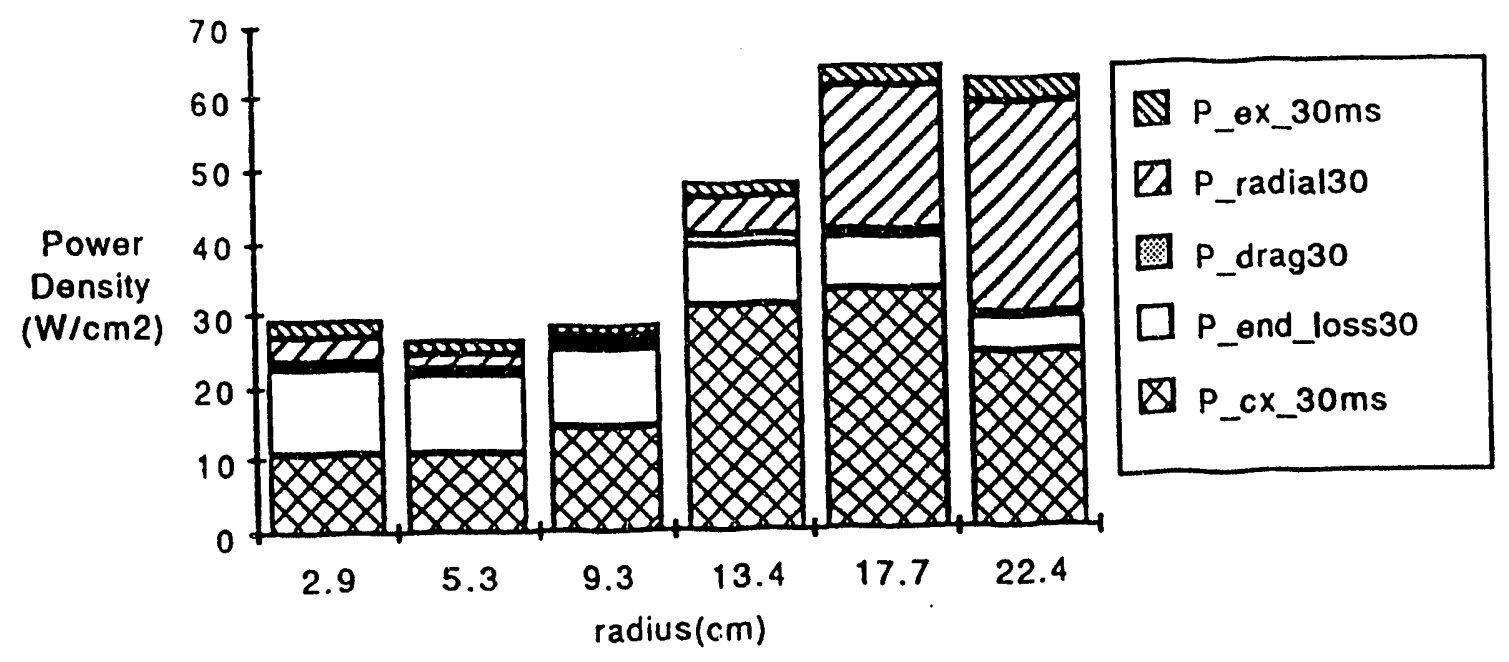

Fig. 6.1-5 Radial Profile of Power Loss Terms for the West Antenna, for Scan Number 5

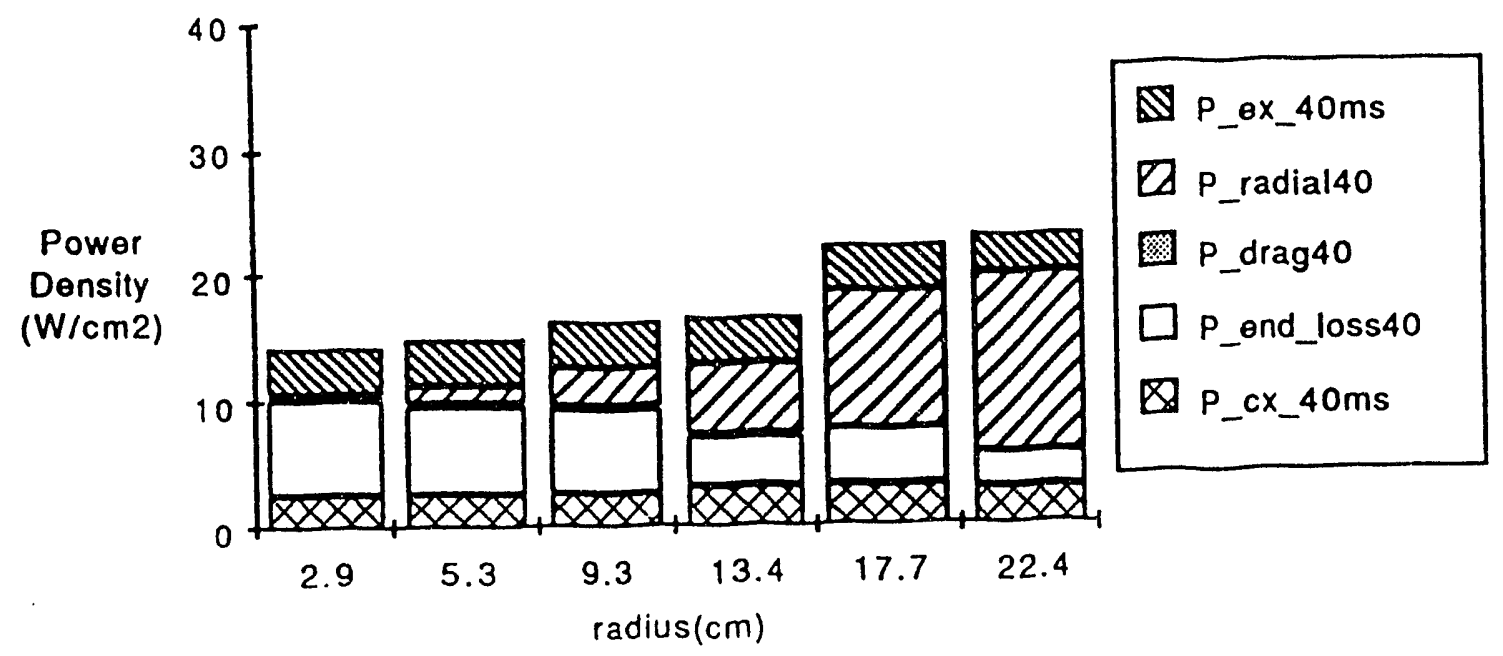

Fig. 6.1-6 Radial Profile of Power Loss Terms with no ICRH, for Scan \#5 


\subsection{Direct heating of electrons by ICRF in mirror machines}

\section{A. W. Molvik}

The first observation of direct heating of electrons by ICRF on mirror machines was made on Phaedrus where the electron heating was greater than could be accounted for by electron drag. ${ }^{6}$ More thorough studies were made by Golovato, et al., 7 on the TARA tandem mirror. Four arguments supported direct electron heating: (1) $\mathrm{T}_{\mathrm{e}}$ was highest when $\mathrm{T}_{\mathrm{i}}$ was low during ICRF heating, so there could be little drag heating. (2) The slow wave, which was the identified mode, would have $k \|=0.04 \mathrm{~cm}^{-1}$ which would provide $v$ thermal $=v_{\text {phase }}$ for efficient Landau damping on the $80 \mathrm{eV}$ electrons. (3) The $\mathrm{T}_{\mathrm{e}}$ radial profile was hollow when ICRF codes predicted small $\mathrm{E}_{\mathbf{Z}}$ on axis, i.e. little electron heating predicted on axis. (4) The electron power balance required several times the estimated drag power, $P_{d r a g}=15 \mathrm{~kW}$. The electron loss power was estimated to be $70-80 \mathrm{~kW}$ from the sum of end-loss power and ionization power, which was independently corroborated from the $\mathrm{T}_{\mathrm{e}}$ decay rate when the plug $\mathrm{ECH}$ was turned off. The difference gives the direct electron heating power of 55-65 $\mathrm{kW}$.

6 R. A. Breun, et al., in Proc. 8th Internat'l Conf. on Plasma Physics and Controlled Fusion Research, Brussels, 1980 (IAEA, Vienna, 1981) p. 105.

7 S. Golovato, et al., "Plasma Production and Heating by ICRF in TARA," Phys. Fluids 31, 3744 (1988). 


\subsection{Bench-Marking the ANTENA Code}

A. W. Molvik

The ANTENA code 8 has been used extensively in predicting and analyzing ICRF heating experiments on mirror machines. Good agreement with plasma loading resistance measurements was found in the TMX-U facility over a range of densities, Fig. 1, and frequencies, Fig. 2, when the ANTENA code modeled the magnetic field strength at the antenna, rather than at the cyclotron resonance. ${ }^{9}$

A more detailed comparison with the ANTENA code was made on the Phaedrus-B tandem mirror, Fig. 3, by Intrator, et al. ${ }^{10}$ They bench-marked the code with magnetic probes to measure the three orthogonal components of the rf magnetic field, Fig. 4. They obtained good agreement between the computed and measured vacuum fields, especially on the side of the antenna away from the gas box baffle, Fig. 5. The antennas were double half-turn loops, arranged in two pairs, one of each pair was rotated $90^{\circ}$ to allow launching rotating fields, Fig. 3.

Axially phased antennas launching fast waves at $\omega / \omega_{\mathrm{ci}}=1.15$ launch waves with a predominant $B_{-}$or right-hand-polarization. $\left(B_{+}\right.$or left-handpolarization waves co-rotate with and heat ions.) The measurements and ANTENA predictions are shown in Fig. 6 where B. is plotted vs radius $(a, b)$ and axial position $(c, d)$. The measurements are taken at frequent intervals, the error bars are merely representative. Reasonable agreement, $\pm 25 \%$, is found well inside the limiter radius of $16 \mathrm{~cm}$ radially, and axially the agreement is good within about $90 \mathrm{~cm}$ of the midplane where the magnetic field is uniform, Fig. 3, as assumed in ANTENA.

The fast wave was selectively launched below the cyclotron frequency at $\omega / \omega_{\mathrm{ci}}=0.8$ by $90^{\circ}$ phasing of the rotating field array. The agreement, Fig. 7 , is somewhat better than that shown in Fig. 6 .

The comparison for the $\mathrm{B}_{+}$, left-hand-polarization which heats ions, is not as good, yielding $\pm 40 \%$ agreement between ANTENA and the

8 B. McVey, "ICRF Antenna Coupling Theory for a Cylindrically Stratified Plasma," MIT Report PFC/RR-84-13, and "ANTENA User Guide," MIT Report PFC/RR-84-13, Cambridge (1984).

9 A. W. Molvik, et al., Proc. 4th International Symp. on Heating in Toroidal Plasmas, Rome (1984); and G. Dimonte, et al., Nuclear Fusion 27, 1959 (1987).

$10 \mathrm{~T}$. Intrator, et al., "Measurements of Electromagnetic Waves in Phaedrus-B: Bench-Mark test of ANTENA Wave Field Calculations," Nuc. Fusion 29_ 377 (1989). 
measurements. This poorer agreement is attributable to poorer signal to noise ratio in measuring a polarization component which makes up only a few percent of the launched fast wave spectrum but which would be the dominant polarization with slow waves as planned for IDEAL. ANTENA predictions for the scrape-off-layer are about a factor of 2 high, which is attributed to shorting of $\mathrm{E}_{\theta}$ by limiters in the experiment. 


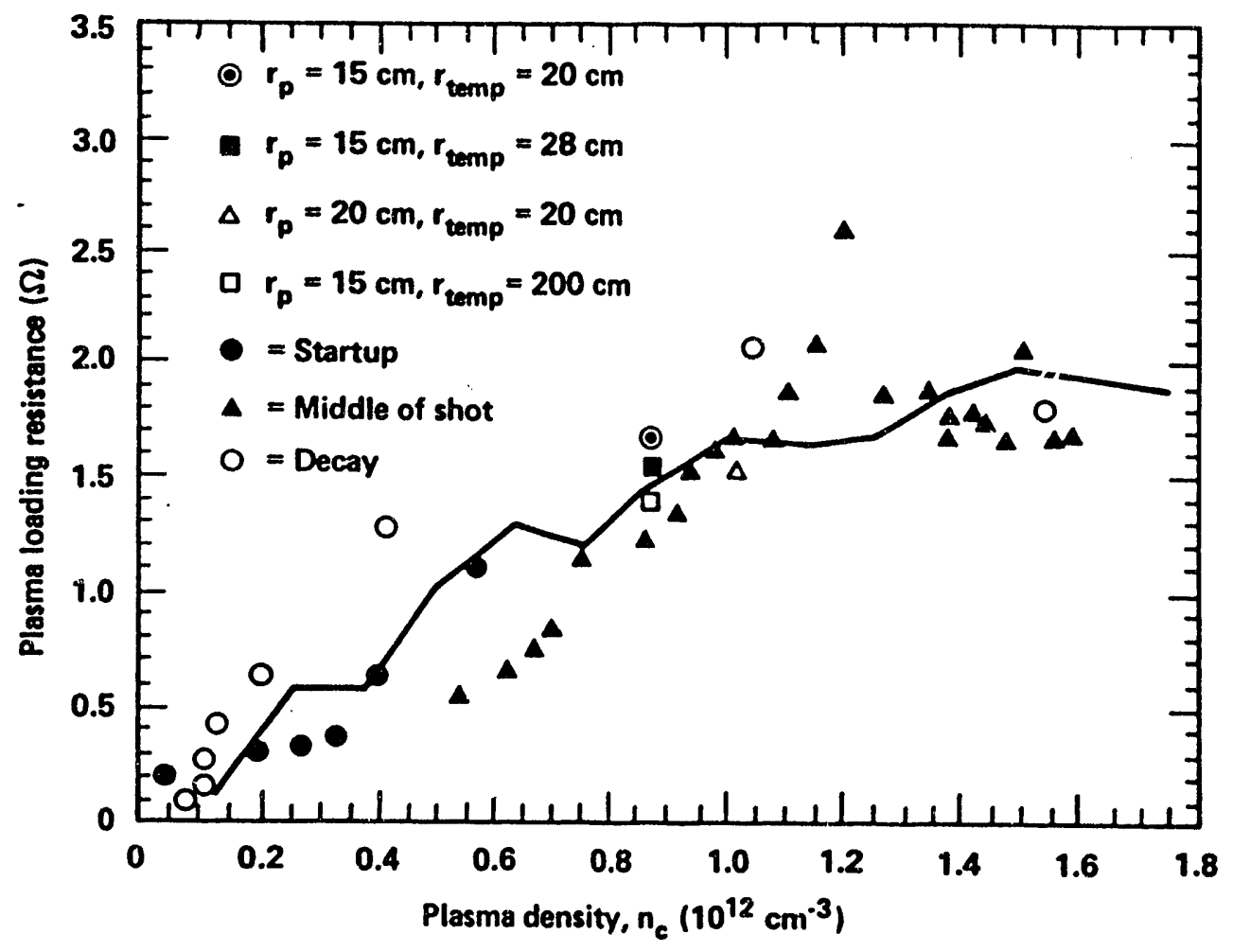

Fig. 6.3-1 The computed loading resistance vs density agrees with measurements at $2.48 \mathrm{MHz}\left(\omega / \omega_{c i}=0.85\right)$. The data are from the $90^{\circ}, 5.5$-turn antenna. 

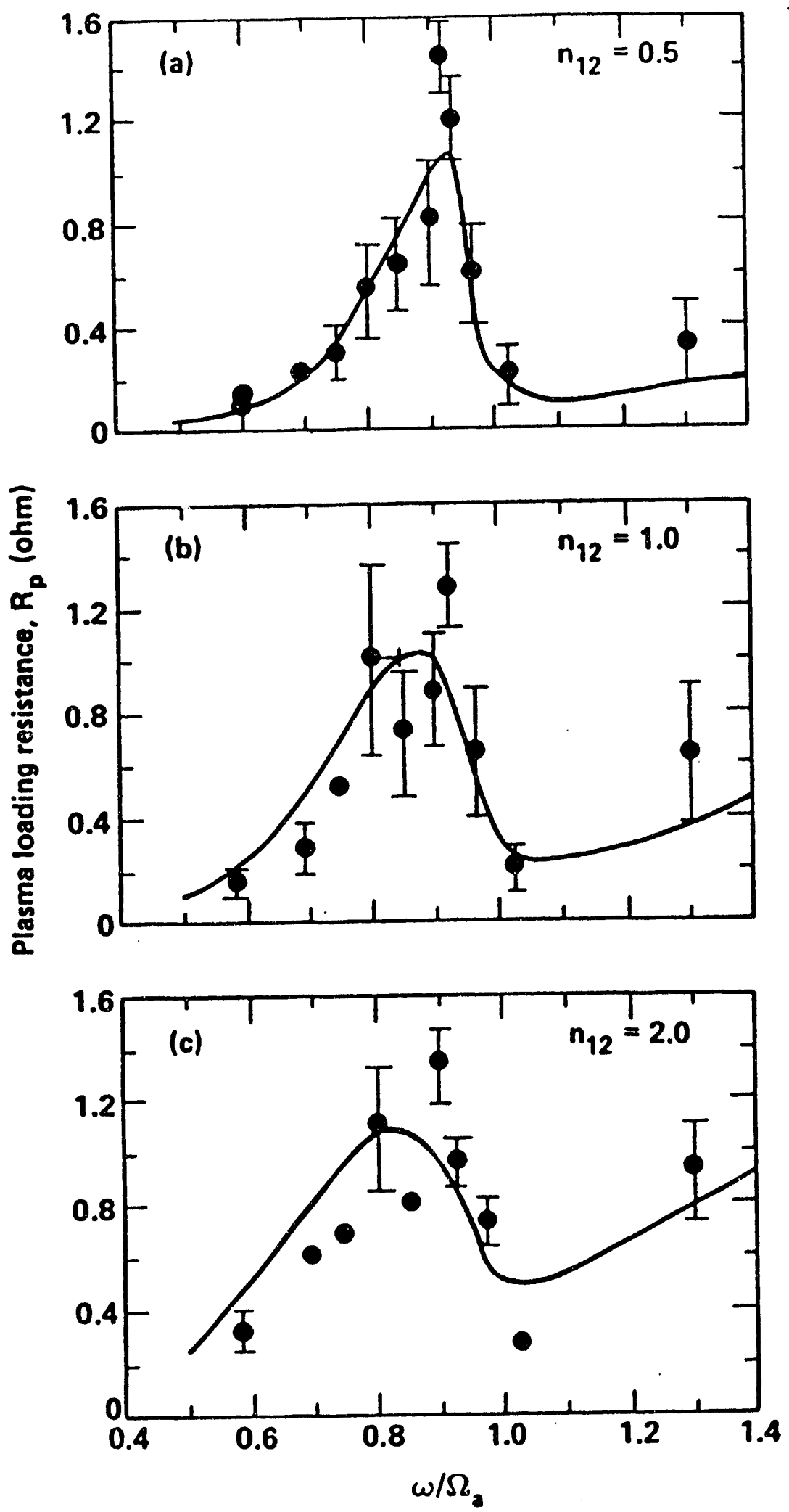

Fig. 6.3-2 Plasma loading resistance versus frequency normalized to ion cyclotron frequency at antenna for selected densities. Lines are ANTENA calculations. 


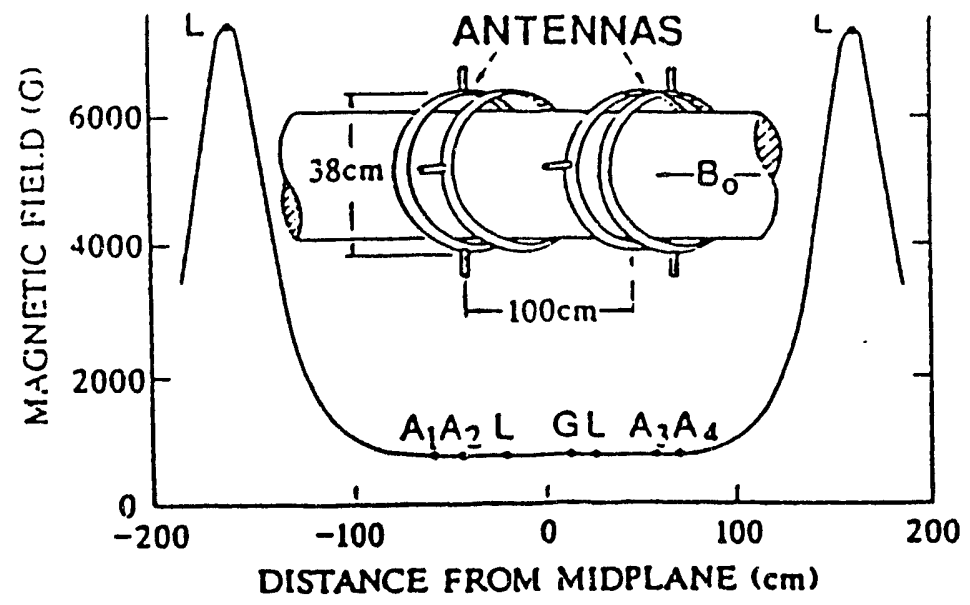

Fig. 6.3-3 Schematic of phased antenna arrays in the central cell. with the axial magnetic field superposed. Shown are the axial locations of the dual half-tum antennas $A I(z=-53.75 \mathrm{~cm})$. $A 2(z=-46.25 \mathrm{~cm}), A 3(z=+46.25 \mathrm{~cm})$ and $A 4(z=+53.75 \mathrm{~cm})$, the limiters $(L)$ and the gas box $(G)$.

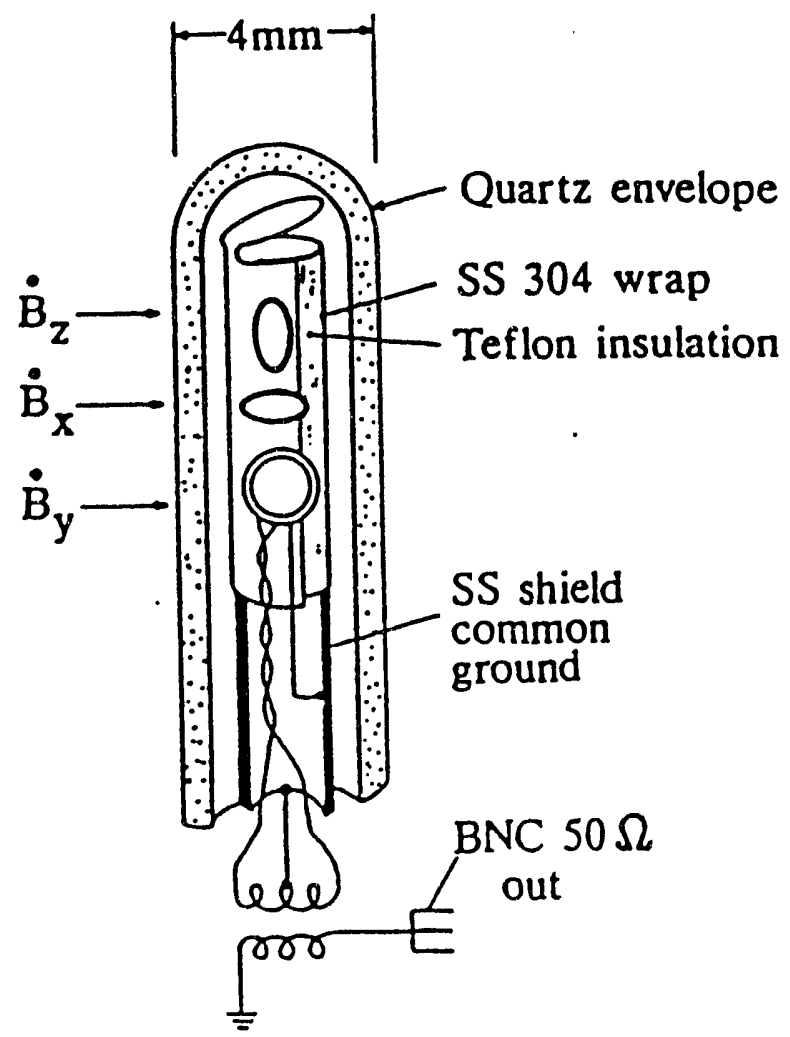

Fig. 6.3-4 Schematic of a typical $\dot{\bar{B}}$ probe, showing 40-turn centre tapped, vector sense coils. The shielding, with a slit at the probe tip, and the centre tapped transformer coupling reject commonmode capacitive coupling to electrostatic noise. 


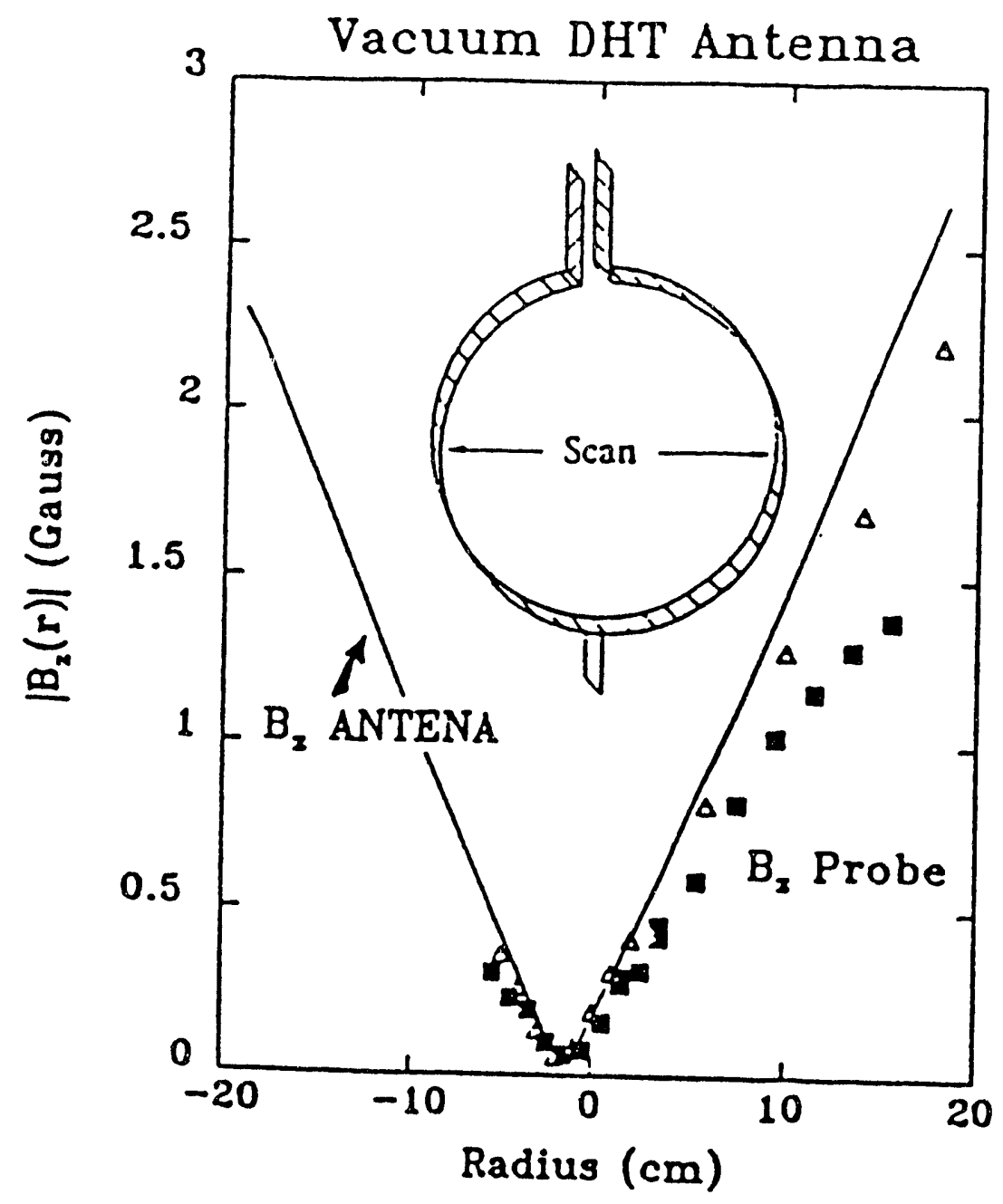

Fig. 6.3-5 Radial profile of the axial $R F \vec{B}_{z}(r)$ field (triangles, $500 \mathrm{~A}$ excitation current) on the outboard side of the antenna in the vacuum. The antenna is indicated by the inset. The predicted ANTENA behaviour is shown by the solid line. Squares (750 A current, data rescaled at 500/750 A for comparison) show the data obtained under she same conditions, but for the magnetic field inboard of the antenna, close to the gas box baffle. 


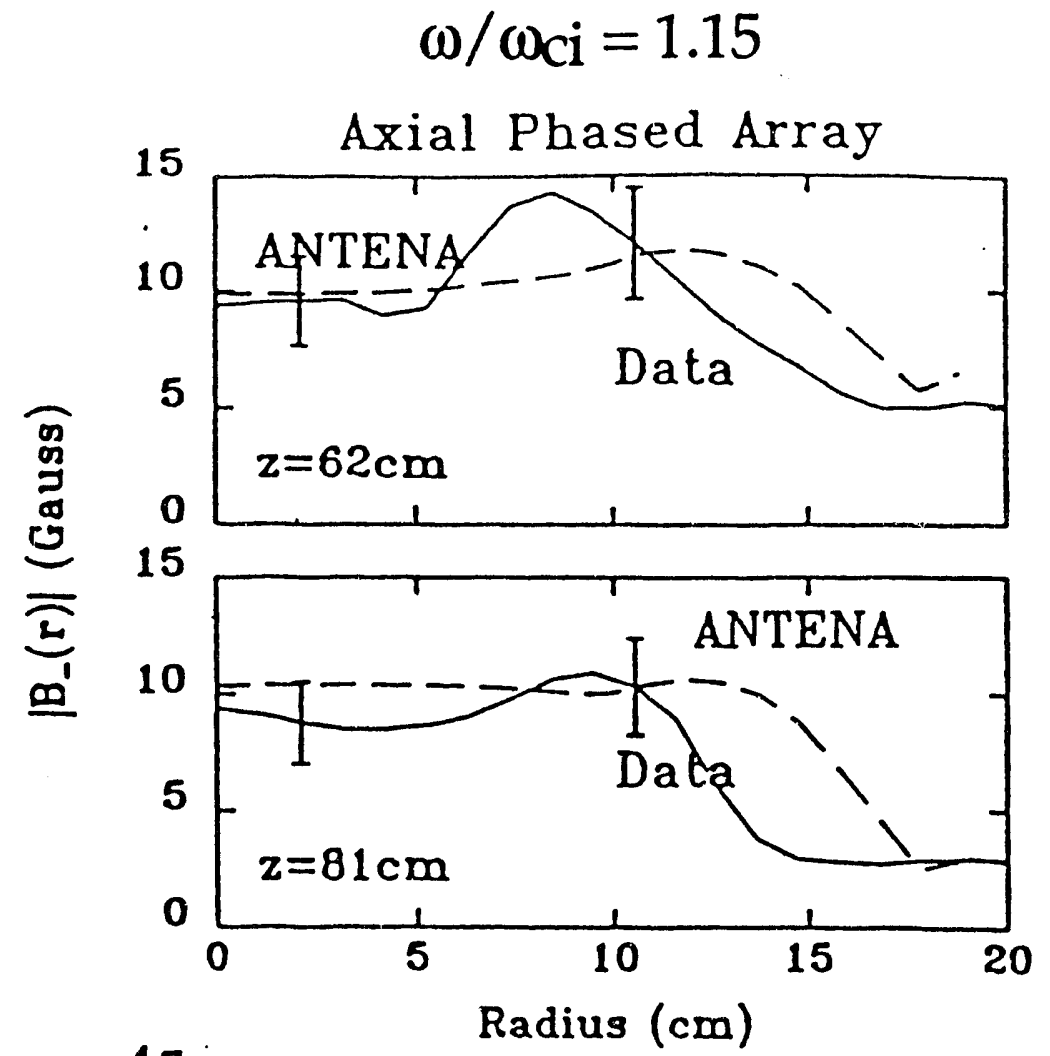

(a)

(b)

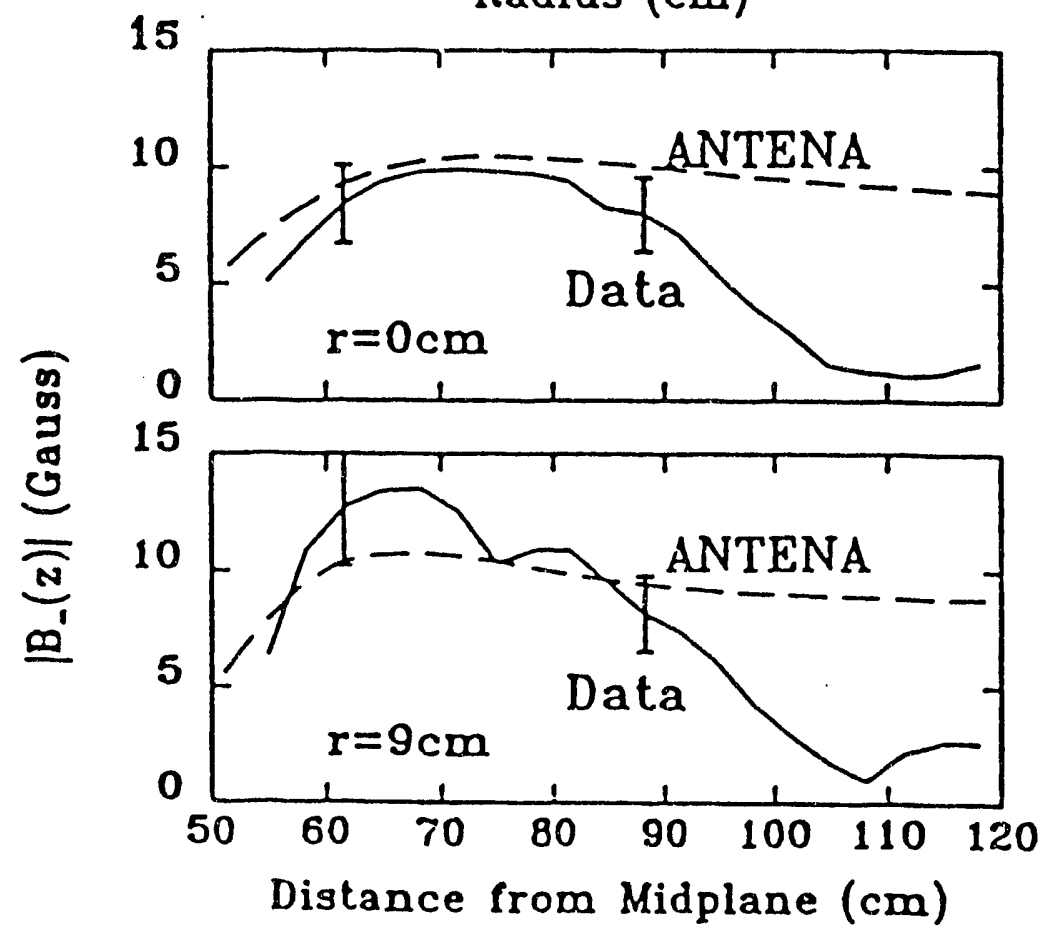

(c)

(d)

Fig. 6.3-6 Radial and axial slices of $B_{-}(r, z)$ contours for the axial antenna phased array, showing a quantitative comparison of the data (solid lines with representative $\pm 20 \%$ error bars) with the code results (dashed line).

$(a, b)$ radial sections for near-field and far-field regions;

(c.d) axial sections for on-axis and off-axis cuts. 
IN'TRATOR et al.

$$
\omega / \omega_{c i}=0.8
$$

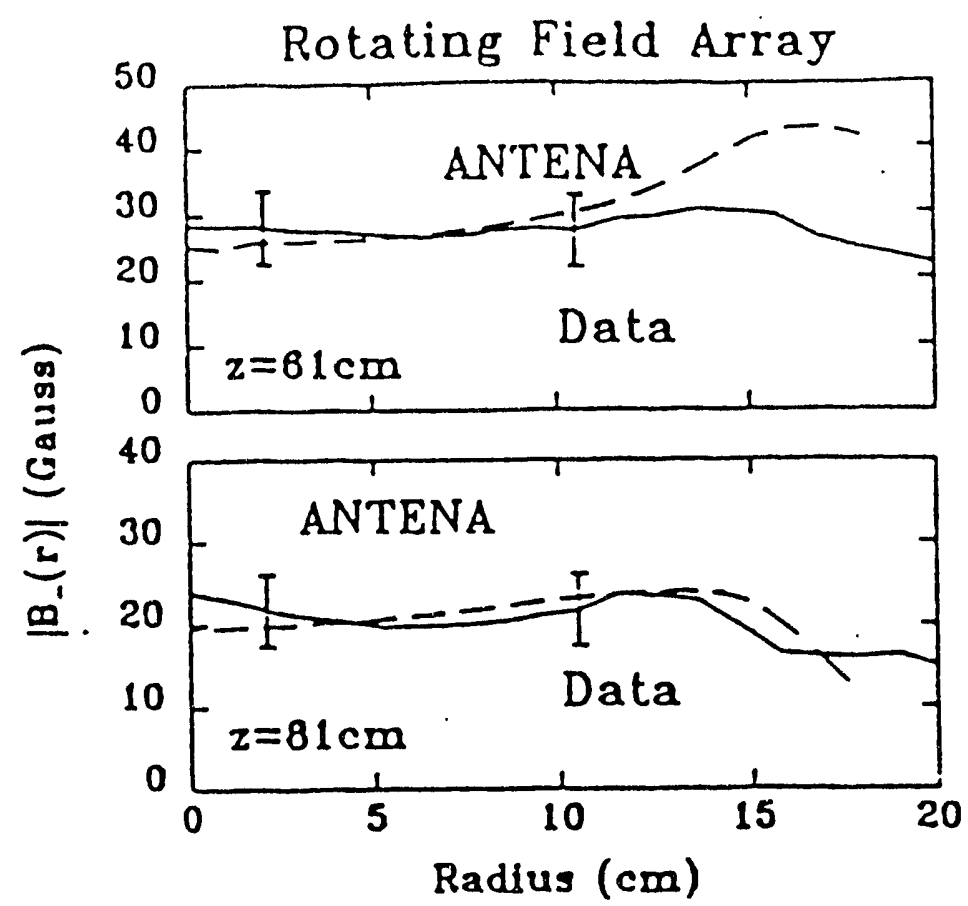

(a)

(b)

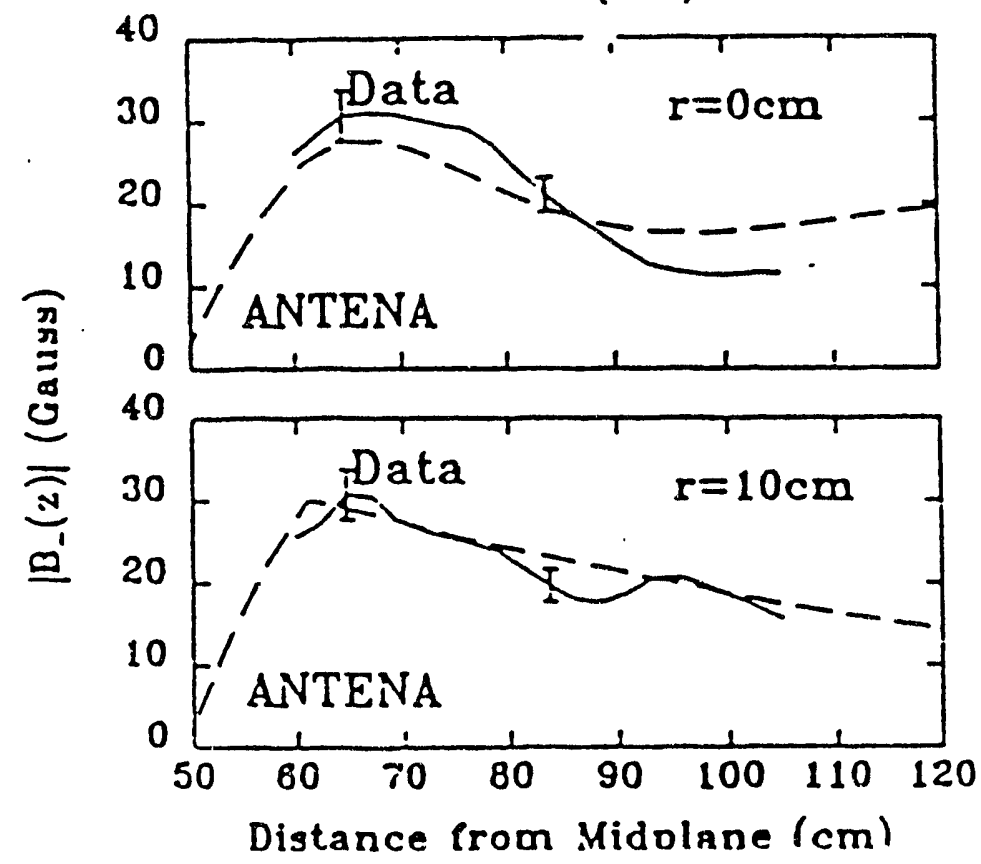

(c)

Fig. 6.3-7 Radial and axial slices of $B_{-}(r, z)$ contours for the rotating field array, showing a quantitative comparison of the data (solid lines with representative $\pm 20 \%$ error bars) with the code results (dashed lines).

$(a, b)$ radial sections for the near-fiold and far-field regions;

(c, d) axial sections for on-axis and off-axis cuts. 


\section{Plasma Start-up}

A. W. Molvik

Startup of plasmas in mirror machines has been accomplished by a variety of methods. Once adequate fueling, heating, and stabilization methods were developed, ${ }^{1}$ startup ceased to be a significant issue as will be discussed below.

Preionization and plasma startup using a plasma gun on axis was first demonstrated successfully on the neutral beam sustained 2XIIB, Fig. 1a. ${ }^{2}$ Subsequently a transition from gun to gas injection was demonstrated on 2XIIB that allowed the plasma to be sustained beyond the plasma gun duration, Fig. 1b. ${ }^{3}$ A similar technique was used on $\mathrm{TMX}^{4}$ and on early Phaedrus tandem mirror experiments, 5 as well as on the thermal barrier tandem mirror GAMMA-10.6 The Phaedrus experiments were the first to demonstrate sustaining a plasma solely by ICRF heating and gas fueling as is planned in IDEAL, after a short preionization pulse from a plasma gun, Fig. 2.

ECH, Electron Cyclotron Heating, breakdown of gas as a preionization, target plasma, technique was developed in a series of experiments in mirrors. ${ }^{7}$ Its use in a neutral-beam sustained mirror was first demonstrated in TMX-U, Fig. 3, where ECH was present because it was an essential ingredient in formation of thermal barriers. ${ }^{8}$ Similar techniques were used on the thermal barrier tandem mirror TARA, ${ }^{9}$ where ICRF heating of the central cell was used to sustain the plasma, and on the Phaedrus tandem mirror which used either a plasma gun or ECH preionization. ${ }^{10}$ Evidence is

1 F. H. Coensgen, et al., 'Stabilization of Neutral-Beam-Sustained, Mirror-Confined Plasma,' Phys. Rev. Lett. 35, 1501, (1975).

2 F. H. Coensgen, et al., "Startup of a Neutral-Beam-Sustained Plasma in a Quasi-dc Magnetic Field," Phys. Rev. Lett. 37, 140, (1976).

3 B. G. Logan, et al., "High-B, Gas-Stabilized, Mirror-Confined Plasma," Phys. Rev. Lett. 37, 1468, (1976).

4 F. H. Coensgen, et al., "Electrostatic Plasma-Confinement Experiments in a Tandem Mirror System," Phys. Rev. Lett. 44, 1132, (1980).

5 R. Breun, et al., "Experiments in a Tandem Mirror Sustained and Heated Solely by rf," Phys. Rev. Lett. 47, 1833, (1981).

6 M. Inutake, et al., "Thermal Barrier Formation and Plasma Confinement in the Axisymmetrized Tandem Mirror GAMMA 10," Phys. Rev. Lett. 55, 939, (1985).

7 R. J. Colchin, et al., Phys. Fluids 13, 501 (1970).

8 D. P. Grubb, et al., "The:mal-Barrier Production and Identification in a Tandem Mirror," Phys. Rev. Lett. 53, 783, (1984).

9 R. S. Post, et al., "Improved Plasma Startup in the TARA Central Cell," Nuciear Fusion 27, 217 (1987).

10 J. R. Ferron, S. N. Golovato, N. Hershkowitz, and R. Goulding, "Interchange stabilization of an axisymmetric single cell mirror using high-frequency electric fields," Phys. Fluids 30, 1869

(1987). 
presented in Fig. 3, that single pass absorption occurred experimentally in the TMX-U, as shown by the lack of heating or diamagnetism at the second harmonic location as would have resulted from cavity mode heating if a significant fraction of the ECH power had not been absorbed on the first pass. Ray tracing calculations, Fig. 4, show that single pass absorption was expected at the fundamental cyclotron resonance zone.

ICRF breakdown of gas was used successfully on one of the earliest ICRF experiments, B-66,11 but required turning on the ICRF as the magnetic field began to rise from zero. ICRF breakdown of gas was used occasionally on Phaedrus but was not dependable. ${ }^{12}$ ICRF breakdown of gas will probably work in IDEAL with the high power density of tunable ICRF available from multiple independent transmitters and antennas. However, we do not have either a strong experimental or theoretical model that would guarantee successful startup.

We therefore propose to use plasma gun startup of IDEAL with gun inserted to the axis during the initial phase of startup, then withdrawn. We believe that this is the simplest, least expensive, method of startup. The major issue is that the gun must be withdrawn before electrodes are melted by ICRF injected power conducted along plasma. If the electrodes are of $0.3 \mathrm{~cm}$ thick Molybdenum, they will be melted by $>2 \mathrm{~kJ} / \mathrm{cm}^{2}$ incident over a time greater than $0.08 \mathrm{~s}$ thermal skin depth time. This requires that the total power to $4 \mathrm{~cm}$ radius profile be less than $\sim 80 \mathrm{~kW}$ for $1 \mathrm{~s}$, which allows a simple, inexpensive gun withdrawal mechanism, but requires that startup be possible at relatively low power. By using a more complex TFTR probe drive which can be inserted $15 \mathrm{~cm}$ and withdrawn within $100 \mathrm{~ms}$, up to $\sim 800 \mathrm{~kW}$ would be allowed.

Power balance calculations for startup range parameters are in progress. The calculations start at densities in the range of $10^{18} \mathrm{~m}^{-3}$, which is high enough for ICRF to couple enough power to the electrons, either directly through Landau Damping or through electron drag, to sustain the plasma. 5 Preliminary results indicate that withdrawing a gun on a time-scale between 1 $\mathrm{s}$ and $0.1 \mathrm{~s}$ will be acceptable. These results will be documented when they are completed and bench-marked. If the power required to sustain a flowing plasma were to prove to be too much for the gun electrodes to withstand, one alternative would be to form a magnetic mirror cell during startup. This would reduce the required power to the order of $40 \mathrm{~kW} .5$

11 W. M. Hook, F. H. Tenney, M. H. Brennan, H. M. Hill, and T. H. Stix, "Experiments on Ion Cyclotron Waves," Phys. Fluids 4, 1131 (1961).

12 R. Majeski, private communication, May, 1993. 
Another alternative would be to use a different preionization method, such as $\mathrm{ECH}$, but that requires either high frequencies, exceeding $70 \mathrm{GHz}$, or requires starting with a lower magnetic field from either turning down the super-conducting coil currents or from using a copper bucking coil that is briefly pulsed during startup (with adequate shielding of nearby superconducting coils from the $\mathrm{dB} / \mathrm{dt}$.). If ECH were installed on IDEAL for other reasons, then it would be the natural method to use for startup. The selection of startup method can be made on the basis of cost and reliability. 


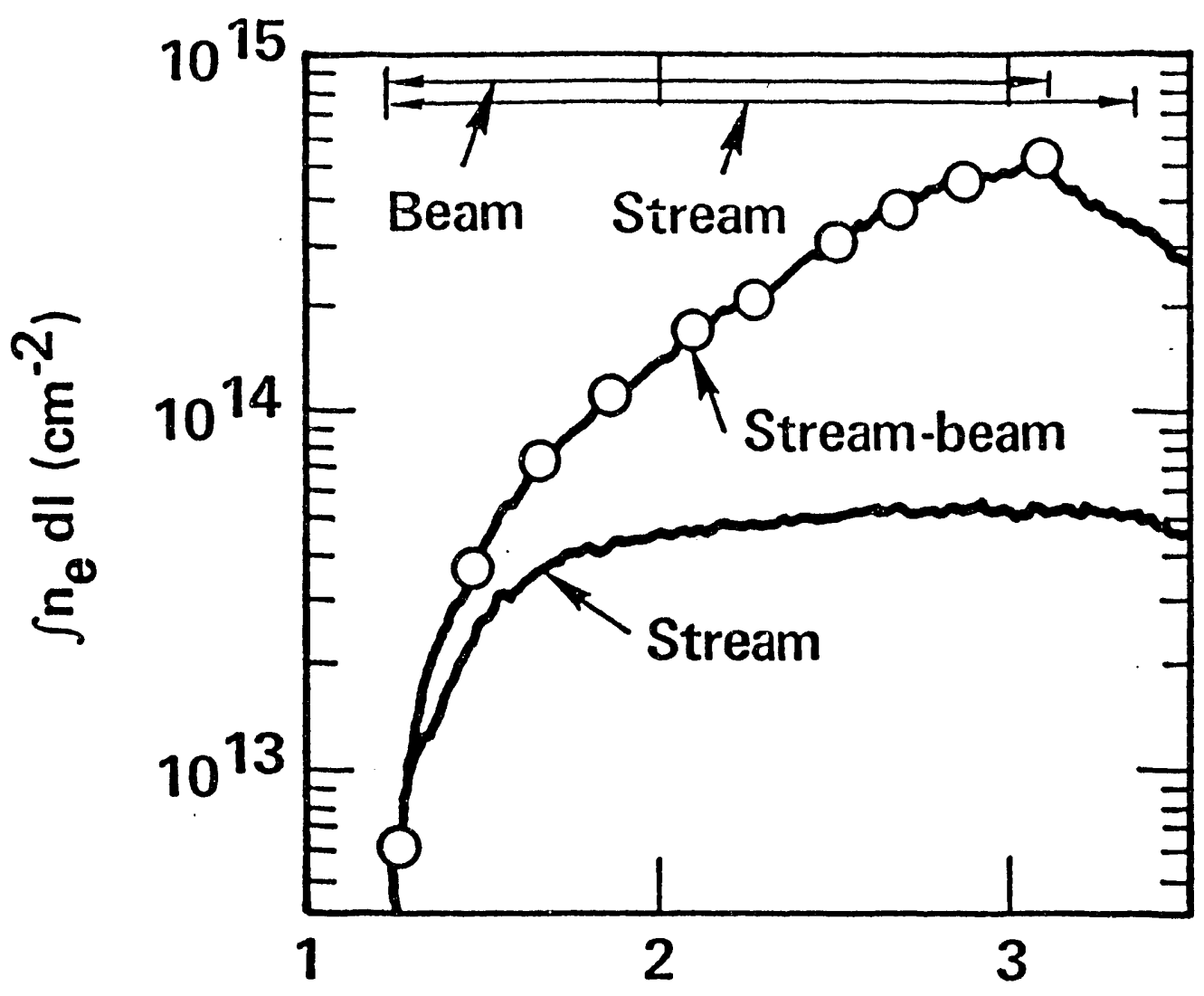

Fig. 7-1a

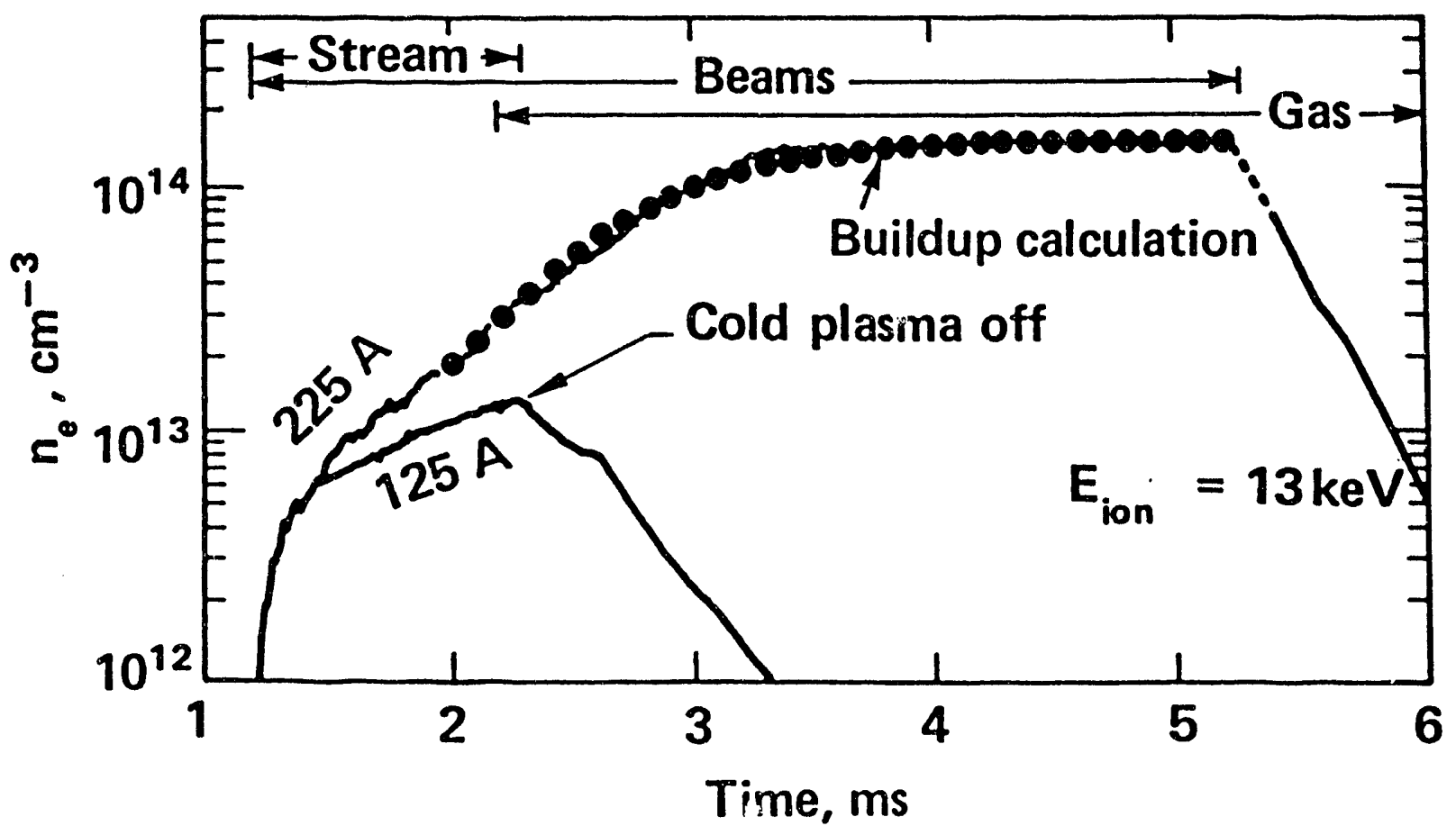

Fig. 7-1b

Fig. 7-1 Density buildup history on streaming plasma target showing (a) $\int n_{c} d \boldsymbol{l}$ with stream guns and (b) $\hat{n}_{\mathrm{c}}$ with 8000 -A hydrogen gas feed. The circular points are computed with the buildup model. 


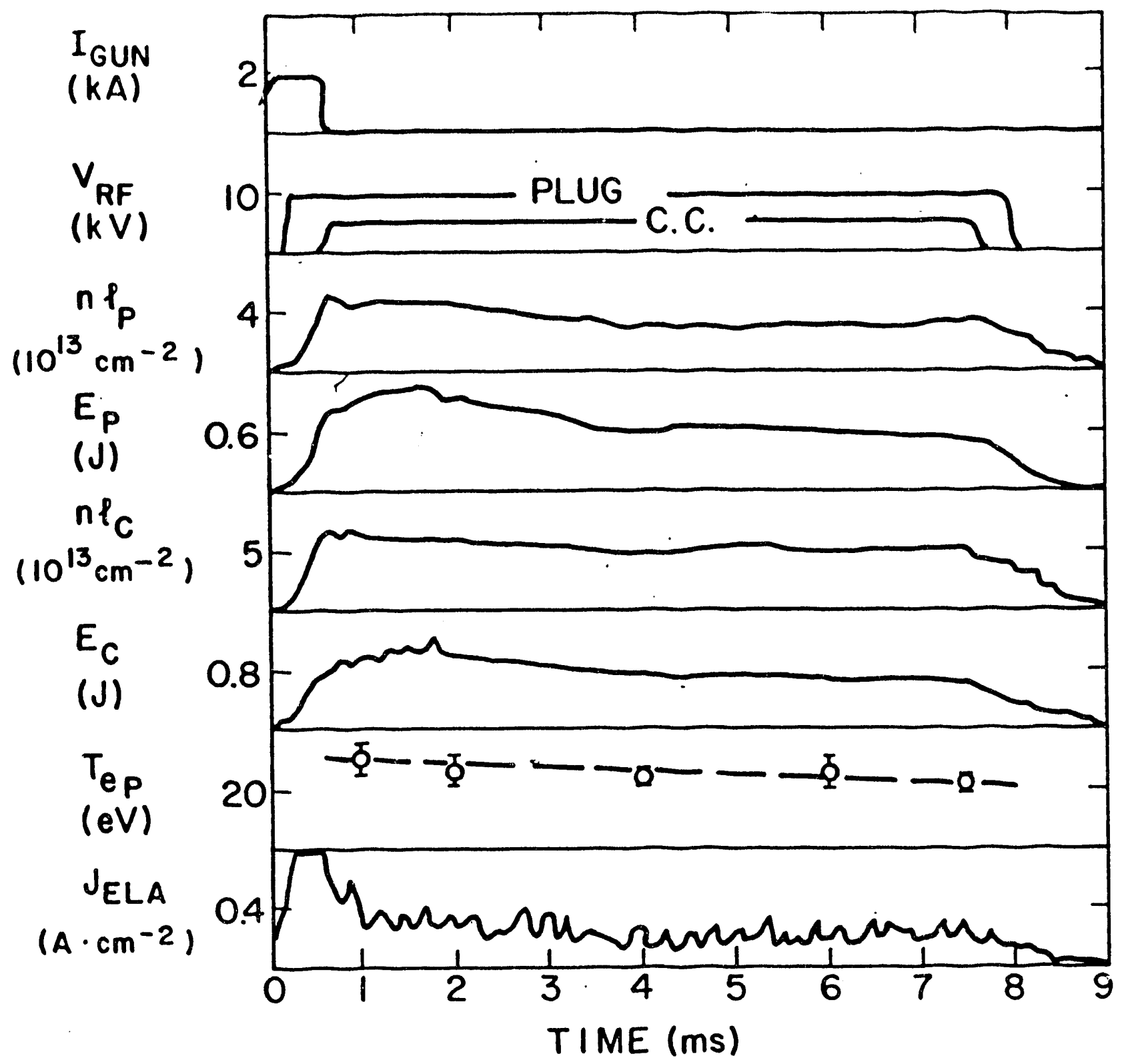

Fig. 7-2 The time evolution of experimental parameters during rf sustained operation. Reading from the top: the plasma gun pulse, the rf heating pulses in the plugs and central cell, the plug line density, the plug energy content, the central-cell line density, the central-cell energy content, the plug electron temperature, and the end-loss current density out of one end near the axis, scaled to the center of the plug. 


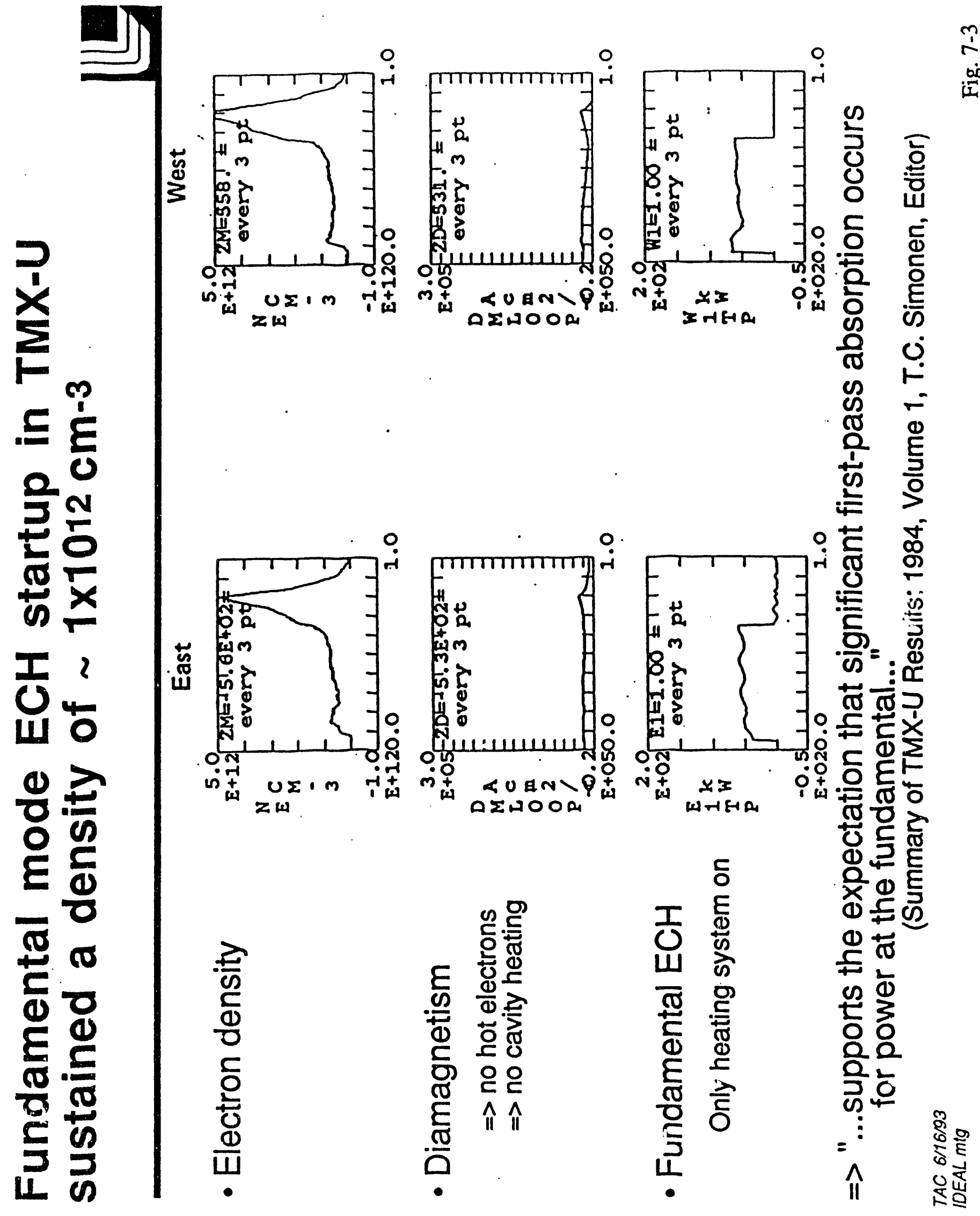




\section{Ray tracing results}

\section{$\Rightarrow>$ illumination of resonance zone}
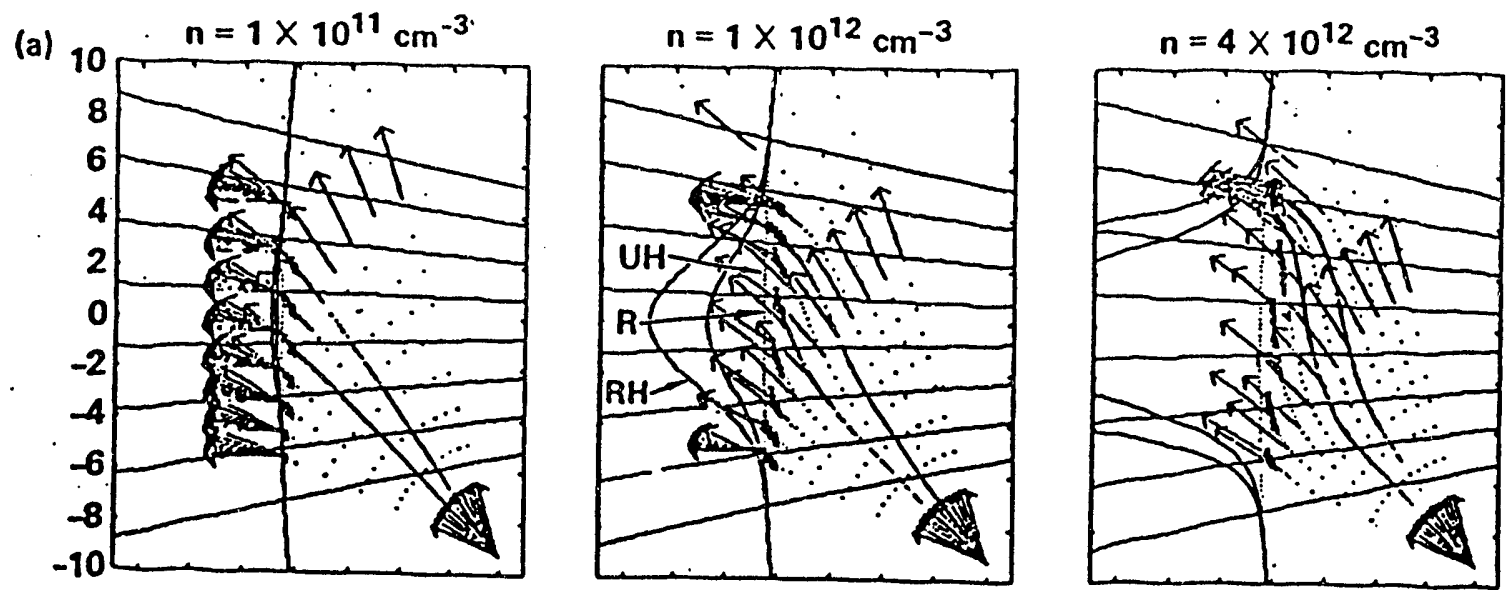

Greater ray bending at higher density $\left(T_{e}=50 \mathrm{eV}\right)$

(b)

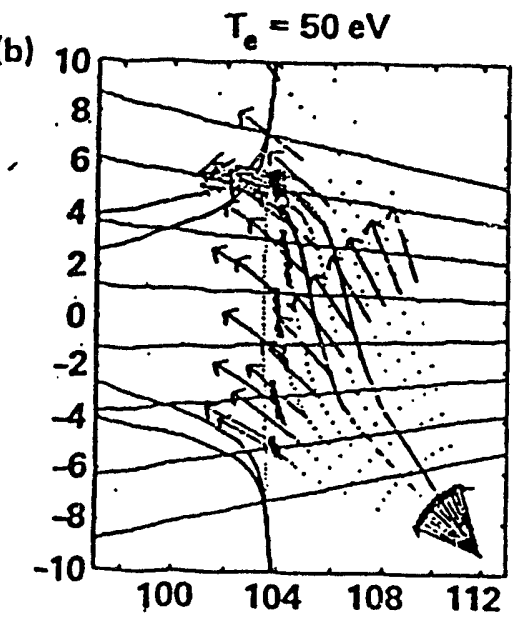

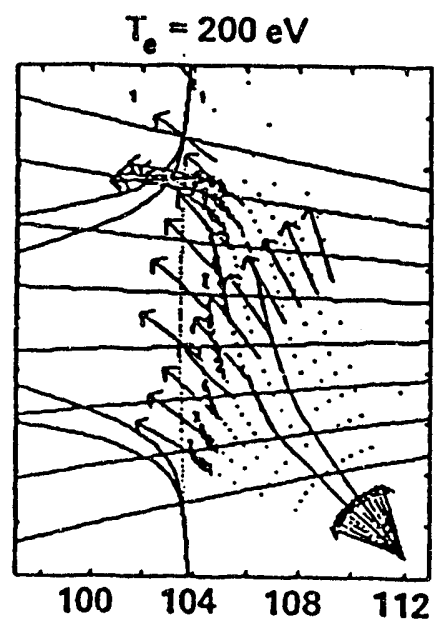

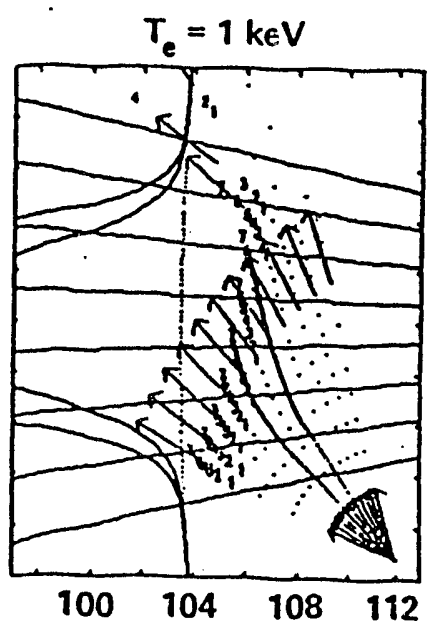

Movement of absorption to $x=0$ at higher $T_{e}\left(n=4 \times 10^{12} \mathrm{~cm}^{-3}\right)$

Fig. 7-4 Ray-tracing calculations in potential peak of TMX-U for extroardinary mode at $\omega=\omega_{c e}$. Surfaces for right-hand $(\mathrm{RH})$, upper hytrid resonance $(U H)$, and electron resonance $(R)$ are shown. Peak antenna power-ray bundles are highlighted. Ray numbers indicate progressive amounts of absorption $(7=99 \%)$. Two parameters were varic $1:$ (a) density at constant $T_{e}=50 \mathrm{eV}$ and (b) electron temperature for constant $n=4 \times 10^{12} \mathrm{~cm}^{-3}$.

TAC $6 / 16 / 93$

IDEAL $\mathrm{mtg}$ 


\section{Limiter Heating}

\section{A. W. Molvik}

Limiter heating will be caused by radial transport of power from the core plasma, as well as by absorption of ICRF power in the scrape-off-layer, SOL.

Radial transport has been addressed by Rognlien in Section 5 . He computed that $8 \%$ of the power would be transported to the limiter at a power density of $50 \mathrm{MW} / \mathrm{m}^{2}$. This is not a severe constraint as steady-state cooling of power densities exceeding $10 \mathrm{MW} / \mathrm{m}^{2}$, particularly over small areas such as the IDEAL limiter, can be handled by technologies such as the Thomson CSF Hypervapotrons, which are used successfully on JET neutral beam and ion dumps to power densities approaching $20 \mathrm{MW} / \mathrm{m}^{2} .{ }^{1}$ A mushroom-shaped cross-section limiter, with an angle of $6^{\circ}$ would reduce the computed power density to $5 \mathrm{MW} / \mathrm{m}^{2}$ well within feasible limits.

Limiter heating would need to be be one of the first issues addressed during the commissioning phase of IDEAL. An infra-red TV camera monitoring limiter temperatures would provide a test of the radial transport computations, and the engineering design, at gradually increasing power levels as confidence is gained. If necessary, the limiter design can be iterated.

Absorption of ICRF power in the scrape-off-layer, SOL, has been indirectly studied, as discussed in Section 6.1. Results from TARA indicated that about $30 \%$ of the power was unaccounted for, ${ }^{2}$ whereas resuits from TMX-U indicated that all the power was accounted for, with an uncertainty of $16 \%{ }^{3}$ We can estimate the power density in the SOL for a maximum of $10 \mathrm{MW}$ launched ICRF power, and make the most conservative assumptions, that IDEAL operates more like TARA than like TMX-U, and that all of the missing $\partial \mathrm{P}=30 \%$, or $3 \mathrm{MW}$ is deposited in the SOL. To handle this power, we assume that each of the 4 antennas has a limiter at either end. Limiters between antennas would be double limiters with independent cooling towards each antenna. Each limiter then has $1 / 8$ of the total SOL power, or about $375 \mathrm{~kW}$ of power to absorb.

Uniform deposition of power over a $4 \mathrm{~cm}$ thick SOL would result in about $25 \mathrm{MW} / \mathrm{m}^{2}$, about half the power level computed for radial transport to limiters. The problem with this apparently acceptable result is that the power

1 G. Duesing et. al., Fusion Technology 11, 141 (1987).

2 S. Golovato, et al., "Plasma Production and Heating by ICRF in TARA," 'ys. Fluids 31, 3744 (1988).

3 S. Falabella, "Measurements of radial profiles of ion cyclotron resonance heating on the Tandem Mirror Experiment Upgrade," Ph.D. Thesis UCRL-53870, 1988. 
is unlikely to be deposited uniformly. The plasma density can be expected to decrease, moving away from the plasma core, in the SOL which will peak the absorbed ICRF power at some radius, probably the limiter edge. Some control over the axial SOL density profile can be obtained by minimizing leakage of gas from the gas box nearest the antennas, and by maximizing the pumping of gas before it reaches the antennas. The solution will lie in conservative engineering of the limiters and their cooling, of the gas box design, and in careful commissioning of the facility in order to measure the peak powers both from radial transport and from absorption in the SOL. A list of allowable operating regimes, with appropriate power limits can thus be developed. 


\section{Engineering and Cost Estimates}

The divertor simulator vessel has been engineered primarily by Grumman for the Princeton Plasma Physics Laboratory (PPPL). No effort has been made to change or alter the design except for filling in gaps or changes caused by our interpretation of the information. The experiment, as envisioned, is presented in the following sections. The assumptions and thought process used in arriving at a benchmark estimate for a generic device is also given.

The estimate given is by no means a final estimate, but should be considered as a check as to the nature of the costs. The estimate can also be used as a reference cost for determining alternatives, or as a base for adding or subtracting relative site credit costs.

\subsection{Engineering Summary}

This section briefly summarizes an interpretation of the PPPL design. The basic parameters are shown in Table 9.1, and can be seen in a view of the machine shown in Figure 9.1.

The vacuum vessel for containing the plasma is composed of 304 stainless steel. This vessel is composed of various subassemblies that when assembled form a vacuum chamber 30 meters long by .5 meters in diameter and .5 inches thick. The vessel contains an inner cooled liner to allow separate control of the plasma facing component temperatures. The heat flux handling capability of the liner is .5 $M W / m^{2}$. The fluid in the liner can also be turned into steam to heat the liner to 150 Degrees $C$ in order to bake the inner plasma facing components.

Eighteen (18) turbomolecular pumps are spread over the length of the vessel. These pumps are backed by roots blower/rotary mechanical pumps. This pumping arrangement provides a pumping speed of 2700 liters/sec at .1 Torr. It is believed this arrangement will provide a base pressure in the low 10-8 Torr range prior to plasma operation. 
Vacuum Vessel

Length

Diamete.

Thickness

Material

Vacuum System

Turbopumps

Pumping Speed

Base Pressure

Magnet System

High Field Solenoids (3.5 T)

Low Field Solenoids $(2.5 \mathrm{~T})$

Solenoid Inner Bore

Drift Quadrupole Coils

Divertor Quadrupole Coils

Ion Cyclotron Heating

Power

Frequency

Antennas

Electron Cyclotron Heating (Optional)

Power

Frequency
30 meters

.5 meter

$1.2 \mathrm{~cm}$

304 Stainless Steel

18

2700 1/sec @.1 Torr

Low $10^{-8}$ Torr range

8 ( 2 coil modules)

22 ( 2 coil modules)

1 meter

2 (4 coil assemblies)

2 (4 coil assemblies)

$8 \mathrm{MW}$

$35 \mathrm{Mhz}$

4

$2 \mathrm{MW}$

$70 \mathrm{Ghz}$

Table 9.1

The plasma in the simulator is contained by 60

superconducting solenoids. These solenoids are manufactured in 30 , 2 coil modules. The coils have an inner bore diameter of 1 meter and completely surround the vacuum vessel. The coils are constructed of Niobium/Titanium wire and are of the pool boiling variety. The center 16 solenoidal coils maintain a field of 3.5 Tesla and the remaining 44 solenoids operate at a field of 2.5 Tesla. This arrangement is called the low field operating mode. A high field option is also being investigated for simulator operation. The coils that are presently being designed do not operate in this regime, and there are no plans to design them for this mode at present. 
At each end of the machine there is a pair of quadrupoles, one in the drift region, and one in the divertor region. These quadrupoles provide stability over the divertor plate, and provide a uniform spot size on the divertor. They are each composed of four modified window frame coils. The coils are connected in series in such a way as to produce a quadrupole field. The coils are composed of hollow core copper conductor which is water cooled to remove heat.

The plasma is primarily heated by ion cyclotron heating methods. The system inputs $8 \mathrm{MW}$ of power at $35 \mathrm{Mhz}$ by way of 4 antennae. Each antenna is composed of a full 5 periods of the wavelength. The antenna will be matched to the plasma with a standard resonant loop/stub stretcher system.

An optional system of heating is also priced. This system uses electron cyclotron heating methods to heat the electrons in the plasma. This system consists of $2 \mathrm{MW}$ of power at $70 \mathrm{Ghz}$ launched into the plasma at the field transition region of the simulator.

A divertor support structure is provided at each end of the simulator. This structure is provided to mount divertor modules under test. The support hardware allows inclination and rotation of the test divertor, and a linear travel of 1 meter along the length of the vessel.

\subsection{Benchmark Assumptions}

In order to periorm any kind of generic estimate for the IDEAL device, some assumpiions must be madie so that it is clear as to what items are included. The assumptions that were used for this estimate are as follows:

1. All estimates are based on "green field" assumptions whenever possible. This assumes no site credits except for those listed in item $\# 2$.

2. The site credits that are assumed for this estimate are that the host site has a building capable of adequate power, LCW, and shielding capabilities for the simulator. 
3. The estimate assumes short runs $\left(<150^{\prime}\right)$ for power and LCW to the facility. If longer runs or increased capabilities are needed this must be added to the estimate.

4. No facility modifications or existing equipment removal costs ane included in the estimate.

5. A stream gun is the method chosen for plasma startup. The stream gun is located on the axis at one end of the machine. The gun can be pulled out of the plasma line of sight within one second to protect the gun from the plasma.

6. The magnet power supply circuits (9) are symmetric about the centerline of the machine. The symmetry is maintained by operating symmetric pairs of coils with the same power supply. The exception is the quadrupole circuits in which each quadrupole of 4 coils is its own circuit.

7. The magnet circuits were derived from the EFFI runs included in the PPPL write-up.

8. An energy dump system is provided for each magnet circuit that contains superconducting magnets. The dump circuit consists of only one resistor in parallel with the coils in the circuit. A DC circuit breaker is provided in each leg of the circuit for transferring the energy from the coils to the resistor. The system might need to be additionally broken down into smaller units upon further design, if the voltage across the coils is deemed to be too excessive.

9. A dummy load is provided for preliminary power supply testing and maintenance.

10. Cabling is used for all coil connections, including jumpers.

11. ICH equipment estimates are based upon recent General Atomic (GA) acquisitions.

12. ECH estimates include Varian gyrotrons and GA corrugated waveguide, miters, and pumpout sections.

13. Facilities estimates, other than cryogenics, are for local power, LCW, and grounding at the vessel only. 
14. Control estimates are based on PLC type controls.

15. A minimum diagnostic set is assumed. This set consists of Thomson Scattering, interferometer, infrared and $\mathrm{H}$-alpha cameras, and their associated controls and data acquisition systems.

16. Nothing has been allocated for control room space, isolated power, UPS, grounding, creature comforts, etc.

17. Costs have been allocated for 6 physics staff positions ( 1 Program Leader, 1 Physics Leader, 4 Physicists) and 2 engineering staff positions (1 Electrical Engineering Leader, 1 Mechanical Engineering Leader) over an assumed 30 month design/construction phase.

18. PACE manpower costs are assumed, $9.7 \%$ purchasing tax, and no other local program or site taxes are used.

\subsection{Benchmark Estimate}

With the assumptions stated in Section 9.2 the following estimate can be presented.

1.0 Vessel

1.1 Tanks w/Baking

$\$ 1820 \mathrm{~K}$

1.2 Supports

$\$ 260 \mathrm{~K}$

1.3 Limiters

$\$ 470 \mathrm{~K}$

1.4 Water Heater

$\$ 100 \mathrm{~K}$

1.5 Liner

$\$ 320 \mathrm{~K}$

1.6 Liner Insulation

$\$ 120 \mathrm{~K}$

1.7 Rails

$\$ 180 \mathrm{~K}$

1.8 Assembly Carts

$\$ 190 \mathrm{~K}$

2.0 Vacuum System

2.1 Roughing System \$130K

2.2 High Vacuum System \$790K

2.3 Gas Fueling

$\$ 340 \mathrm{~K}$ 
2.4 Glow Discharge Cleaning

$\$ 150 \mathrm{~K}$

2.5 Stream Gun (Startup)

$\$ 230 \mathrm{~K}$

$\$ 1640 \mathrm{~K}$

3.0 Magnet System

3.1 High Field Solenoids

$\$ 1840 \mathrm{~K}$

3.2 Low Field Solenoids

$\$ 3500 \mathrm{~K}$

3.3 Drift Quadrupoles

3.4 Divertor Quadrupoles

$\$ 300 \mathrm{~K}$

$\$ 350 \mathrm{~K}$

3.5 Magnet Protection

$\$ 130 \mathrm{~K}$

3.6 Leads

$\$ 1100 \mathrm{~K}$

3.7 Magnet Cases

$\$ 670 \mathrm{~K}$

3.8 LN Manifolds

$\$ 90 \mathrm{~K}$

3.9 Alignment

3.10 End Solenoid

$\$ 190 \mathrm{~K}$

$\$ 0 \mathrm{~K}$

4.0 Magnet Power Supplies

$\$ 8170 \mathrm{~K}$

4.1 High Field Solenoids

4.2 Low Field Solenoids

$\$ 330 \mathrm{~K}$

$\$ 910 \mathrm{~K}$

4.3 Drift Quadrupoles

$\$ 600 \mathrm{~K}$

4.4 Divertor Quadrupoles

$\$ 770 \mathrm{~K}$

4.5 Dummy Load

$\$ 90 \mathrm{~K}$

4.6 End Solenoid

$\$ 0 \mathrm{~K}$

$\$ 2700 \mathrm{~K}$

5.0 Ion Cyclotron Heating (8MW)

5.1 Transmitters

5.2 Feedlines

5.3 Matching Networks

5.4 Antenna

5.5 Building

$\$ 9240 \mathrm{~K}$

$\$ 110 \mathrm{~K}$

$\$ 550 \mathrm{~K}$

$\$ 730 \mathrm{~K}$

$\$ 0 \mathrm{~K}$

$\$ 10630 \mathrm{~K}$

6.0 Divertor

6.1 Support Hardware

$\$ 190 \mathrm{~K}$

7.0 Facilities

7.1 Electric Utilities

7.2 HVAC

$7.3 \mathrm{LCW}$

7.4 Cryogenics

7.5 Site Preparation

7.6 Shielding

$\begin{array}{r}\$ 240 \mathrm{~K} \\ \$ 230 \mathrm{~K} \\ \$ 90 \mathrm{~K} \\ \$ 2000 \mathrm{~K} \\ \$ 0 \mathrm{~K} \\ \$ 0 \mathrm{~K} \\ \hline\end{array}$


$\$ 2560 \mathrm{~K}$

8.0 Controls

8.1 Vacuum System

$\$ 90 \mathrm{~K}$

8.2 Magnets

$\$ 170 \mathrm{~K}$

8.3 Cryogenics

$\$ 160 \mathrm{~K}$

8.4 Fueling

$\$ 90 \mathrm{~K}$

$8.5 \mathrm{ICH}$

$\$ 170 \mathrm{~K}$

8.6 Divertor

$\$ 220 \mathrm{~K}$

8.7 Baking

$\$ 90 \mathrm{~K}$

$\$ 990 \mathrm{~K}$

9.0 Diagnostics

9.1 Thomson Scattering

$\$ 690 \mathrm{~K}$

9.2 Interferometer

$\$ 440 \mathrm{~K}$

9.3 IR/HA Cameras

$\$ 300 \mathrm{~K}$

9.4 Balance of Diagnostics

$\$ 620 \mathrm{~K}$

9.5 Controls

$\$ 320 \mathrm{~K}$

9.6 Data Acquisition

10.0 Project Manpower

$\$ 630 \mathrm{~K}$

$\$ 3000 \mathrm{~K}$

10.1 Physics (6 FTE, $30 \mathrm{M}$ )

$\$ 1950 \mathrm{~K}$

10.2 Eng. (2 FTE, $30 \mathrm{M}$ )

$\$ 680 \mathrm{~K}$

$\$ 2630 \mathrm{~K}$

WBS Cost Total

Contingency $25 \%$

Total Project Cost

$\$ 35970 \mathrm{~K}$

$\$ 8993 \mathrm{~K}$

$\$ 44963 K$

11.0 ECH (Optional, 2MW)

11.1 Power Supplies

11.2 Gyrotrons

11.3 Gyrotron Aux Equip

$\$ 3650 \mathrm{~K}$

$\$ 2200 \mathrm{~K}$

11.4 Waveguide

$\$ 1180 \mathrm{~K}$

11.5 Launchers

$\$ 1450 \mathrm{~K}$

11.6 Controls

$\$ 200 \mathrm{~K}$

$\$ 170 \mathrm{~K}$

$\$ 8850 \mathrm{~K}$

Contingency $25 \%$

$\$ 2213 \mathrm{~K}$

Optional ECH Total

\$11063K

Project Cost W/ECH

$\$ 56026 \mathrm{~K}$ 


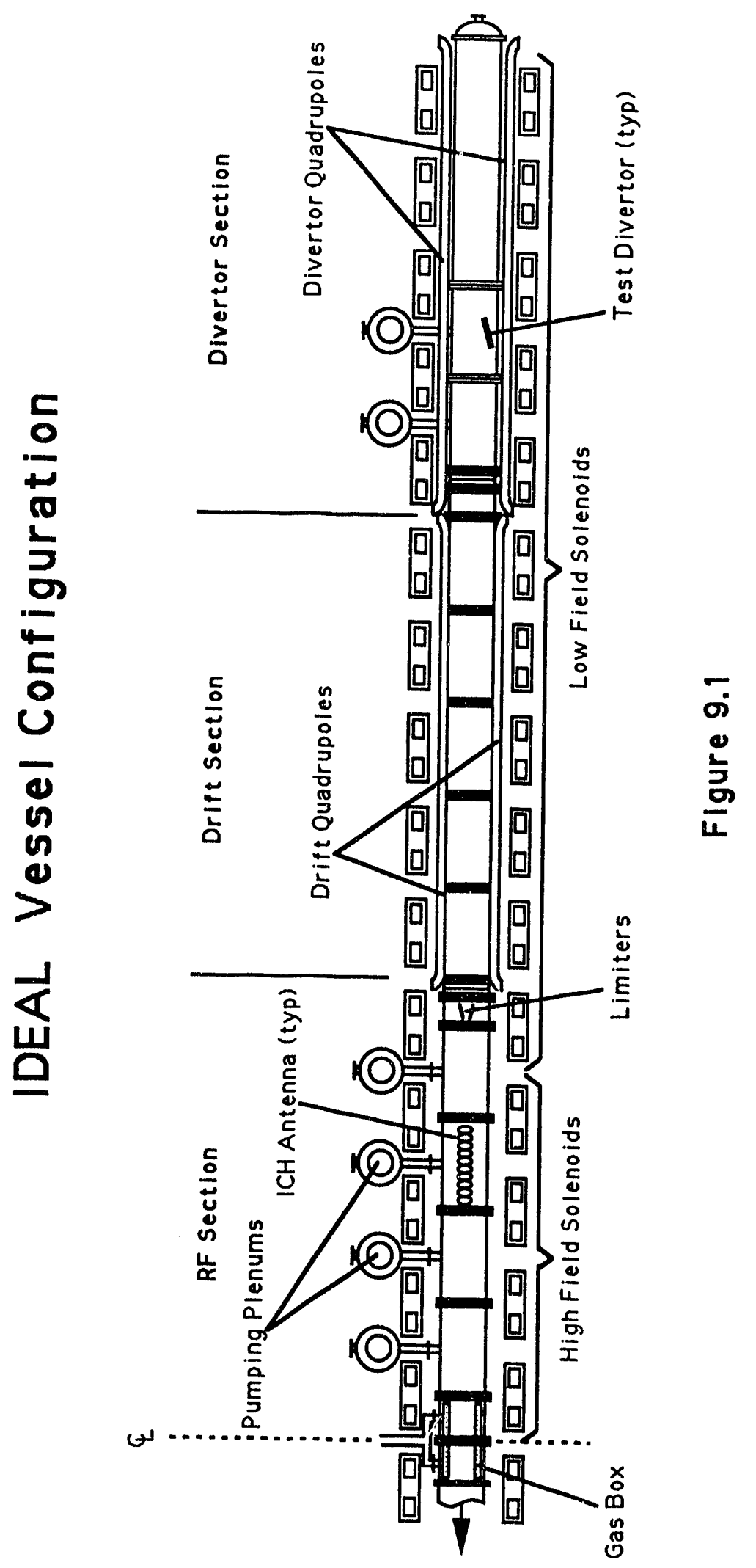



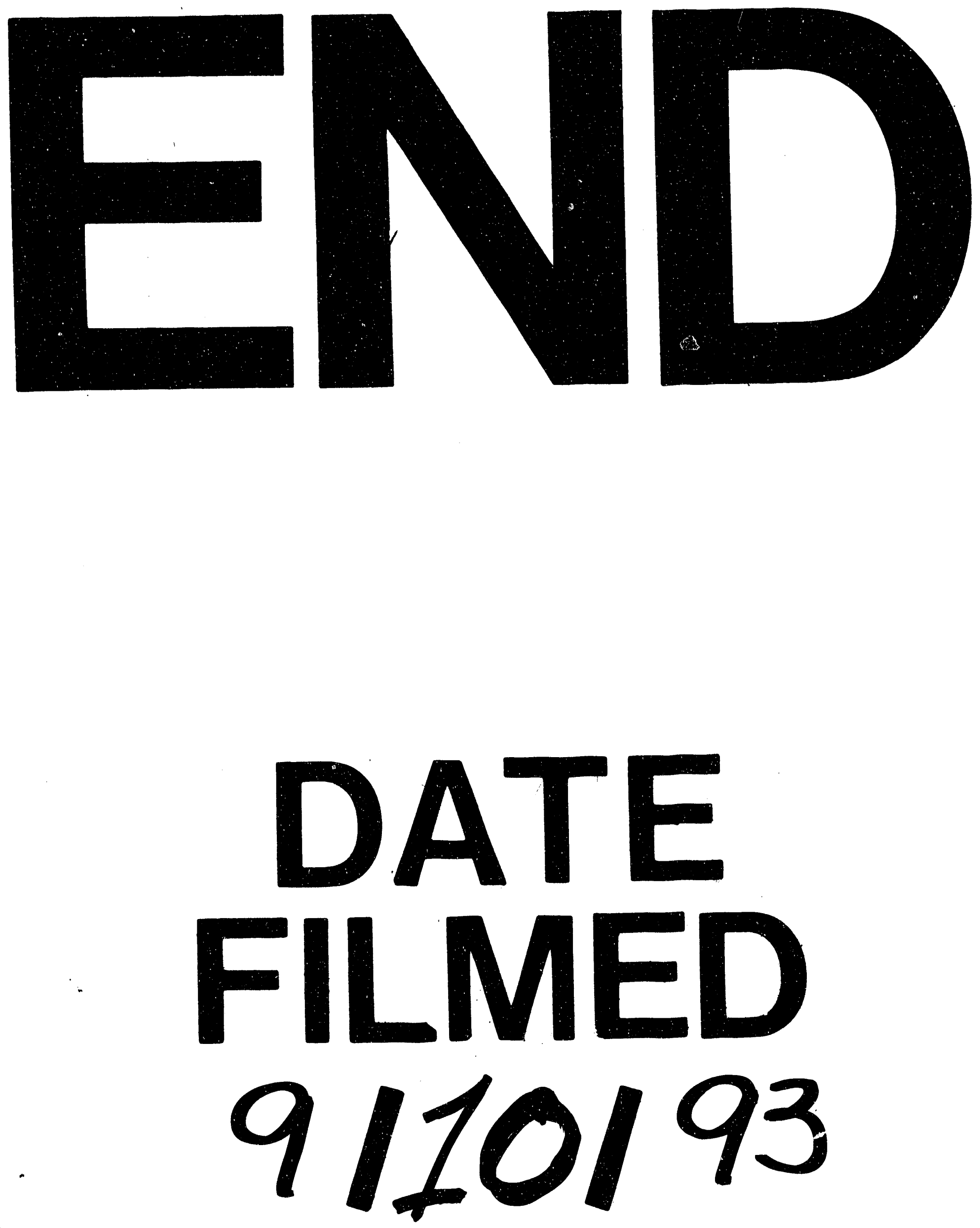
